\title{
Molecular Structures of Magnesium Dichloride Sheets and Nanoballs
}

Tommi N. P. Luhtanen, Mikko Linnolahti, and Tapani A. Pakkanen*

Department of Chemistry, University of Joensuu, P.O. Box 111, FIN-80101 Joensuu, Finland

\section{Supporting Information}

\section{Used basis sets for $\mathrm{Mg}$ and $\mathrm{Cl}$}

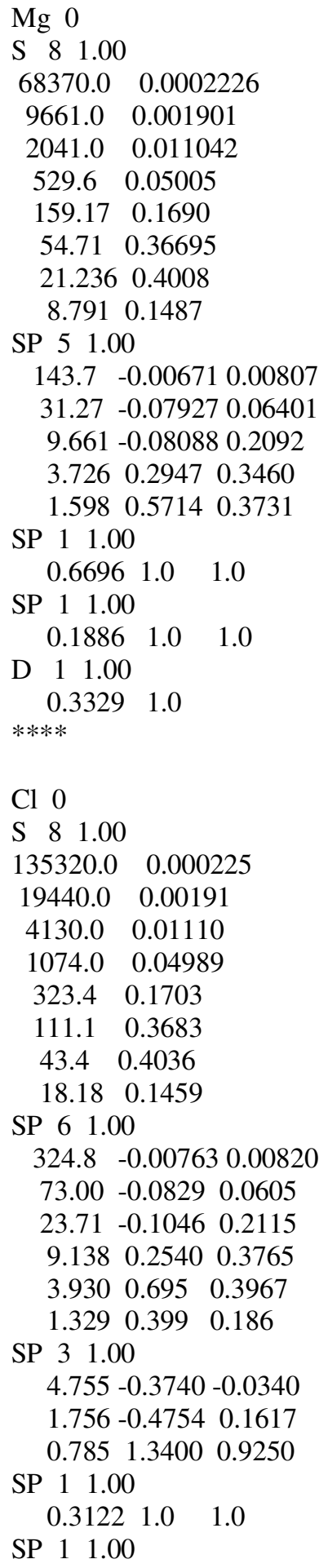


$\begin{array}{lll}0.1453 & 1.0 & 1.0\end{array}$

D 11.00

0.30421 .0

D 11.00

0.11731 .0

$* * * *$

\section{$\mathrm{Mg}_{4} \mathrm{Cl}_{8}$ (Optimized)}

Input Keywords:

\#p opt hf/Gen geom=connectivity scfcyc $=800$

Input coordinates:

$\begin{array}{lccc}\mathrm{Cl} & 0.73353600 & 0.36384200 & -2.99428000 \\ \mathrm{Mg} & -0.45105500 & -0.00297900 & -1.01409800 \\ \mathrm{Cl} & 1.34793500 & 0.04659900 & 0.66749500 \\ \mathrm{Mg} & -0.49106300 & -0.36922500 & 2.43220400 \\ \mathrm{Cl} & -1.93511000 & -0.95537200 & 0.51758500 \\ \mathrm{Cl} & -0.16371700 & -1.80075200 & 4.09084800 \\ \mathrm{Cl} & 0.48339400 & 4.23372200 & -4.27132100 \\ \mathrm{Mg} & 0.81072100 & 2.80219100 & -2.61267500 \\ \mathrm{Cl} & 2.25476700 & 3.38833800 & -0.69805500 \\ \mathrm{Mg} & 0.77071200 & 2.43594100 & 0.83362400 \\ \mathrm{Cl} & -1.02827800 & 2.38636400 & -0.84797000 \\ \mathrm{Cl} & -0.41387800 & 2.06912200 & 2.81380600\end{array}$

Output coordinates:

$\begin{array}{lccc}\mathrm{Cl} & -2.037763 & -2.064168 & -1.041656 \\ \mathrm{Mg} & 0.000146 & -1.656635 & -0.000058 \\ \mathrm{Cl} & 1.030087 & -0.000039 & -1.511460 \\ \mathrm{Mg} & 3.024245 & 0.000141 & 0.134139 \\ \mathrm{Cl} & 2.037859 & -2.064202 & 1.041697 \\ \mathrm{Cl} & 5.104903 & 0.000035 & -0.582753 \\ \mathrm{Cl} & -5.104904 & -0.000035 & 0.582753 \\ \mathrm{Mg} & -3.024245 & -0.000141 & -0.134138 \\ \mathrm{Cl} & -2.037859 & 2.064202 & -1.041697 \\ \mathrm{Mg} & -0.000146 & 1.656635 & 0.000058 \\ \mathrm{Cl} & -1.030087 & 0.000040 & 1.511460 \\ \mathrm{Cl} & 2.037763 & 2.064168 & 1.041656\end{array}$

\section{$\mathrm{Mg}_{4} \mathrm{Cl}_{8}$ (Frozen)}

Input Keywords:

$\# p$ hf/Gen geom=connectivity scfcyc $=800$

Input coordinates:

$\begin{array}{lccc}\mathrm{Cl} & -2.21636232 & 1.14758937 & -1.36300000 \\ \mathrm{Cl} & -2.21636232 & 4.78858937 & -1.36300000 \\ \mathrm{Cl} & -0.11436232 & -2.49341063 & 1.36300000 \\ \mathrm{Mg} & -1.16536232 & -0.67241063 & 0.00000000 \\ \mathrm{Cl} & -0.11436232 & 1.14758937 & 1.36300000 \\ \mathrm{Cl} & 0.93663768 & -0.67241063 & -1.36300000 \\ \mathrm{Mg} & -1.16536232 & 2.96858937 & 0.00000000 \\ \mathrm{Cl} & 0.93663768 & 2.96858937 & -1.36300000 \\ \mathrm{Cl} & 3.03863768 & -4.31441063 & 1.36300000 \\ \mathrm{Mg} & 1.98763768 & -2.49341063 & 0.00000000 \\ \mathrm{Cl} & 3.03863768 & -0.67241063 & 1.36300000 \\ \mathrm{Mg} & 1.98763768 & 1.14758937 & 0.00000000\end{array}$

$\mathrm{Mg}_{6} \mathrm{Cl}_{12}$ (Optimized) 
Input Keywords:

\#p opt hf/Gen geom=connectivity scfcyc $=800$

Input coordinates:

$\begin{array}{lccc}\mathrm{Cl} & 0.28851000 & -4.23933100 & -2.15690000 \\ \mathrm{Mg} & 0.41032700 & -2.00544600 & -1.52100100 \\ \mathrm{Cl} & 0.63901400 & -2.53958900 & 0.85045700 \\ \mathrm{Mg} & -0.10082500 & -4.92124100 & 0.18717800 \\ \mathrm{Mg} & -1.83380800 & -1.89444500 & 1.09566600 \\ \mathrm{Cl} & 1.13867300 & 0.19383400 & -1.94615700 \\ \mathrm{Cl} & -1.88867200 & -1.19769000 & -1.29332600 \\ \mathrm{Mg} & -0.81515800 & 1.18051800 & -0.86865500 \\ \mathrm{Cl} & 0.96876900 & -6.66993500 & 1.03806300 \\ \mathrm{Mg} & -2.54825600 & 4.20729300 & 0.03902300 \\ \mathrm{Cl} & -2.31427800 & -4.21319700 & 0.87753100 \\ \mathrm{Mg} & -3.05741100 & 1.29230000 & 1.75001000 \\ \mathrm{Cl} & -0.33557600 & 3.49968500 & -0.65344900 \\ \mathrm{Cl} & -0.75881300 & 0.48443100 & 1.52010300 \\ \mathrm{Cl} & -3.28858500 & 1.82519700 & -0.62163100 \\ \mathrm{Cl} & -3.78533200 & -0.90719600 & 2.17597500 \\ \mathrm{Cl} & -3.62019900 & 5.95513600 & -0.81067600 \\ \mathrm{Cl} & -2.93494100 & 3.52645500 & 2.38439000\end{array}$

Output coordinates:

$\begin{array}{lccc}\mathrm{Cl} & -4.214212 & -1.903096 & -1.167017 \\ \mathrm{Mg} & -2.101453 & -1.945134 & -0.222008 \\ \mathrm{Cl} & -2.661718 & -0.245337 & 1.445070 \\ \mathrm{Mg} & -4.719861 & 0.230608 & -0.045070 \\ \mathrm{Mg} & -1.335314 & 1.392206 & -0.034672 \\ \mathrm{Cl} & -0.227472 & -3.206925 & 0.416682 \\ \mathrm{Cl} & -0.696800 & -0.459655 & -1.543229 \\ \mathrm{Mg} & 1.335292 & -1.392205 & 0.034753 \\ \mathrm{Cl} & -6.702344 & 0.527688 & 0.870635 \\ \mathrm{Mg} & 4.719847 & -0.230573 & 0.045062 \\ \mathrm{Cl} & -3.413626 & 2.047858 & -0.958103 \\ \mathrm{Mg} & 2.101443 & 1.945137 & 0.221893 \\ \mathrm{Cl} & 3.413606 & -2.047779 & 0.958222 \\ \mathrm{Cl} & 0.696832 & 0.459717 & 1.543237 \\ \mathrm{Cl} & 2.661748 & 0.245281 & -1.445089 \\ \mathrm{Cl} & 0.227454 & 3.206907 & -0.416786 \\ \mathrm{Cl} & 6.702363 & -0.527862 & -0.870508 \\ \mathrm{Cl} & 4.214201 & 1.903177 & 1.166916\end{array}$

\section{$\mathrm{Mg}_{8} \mathrm{Cl}_{16}$ (Optimized)}

Input Keywords:

\#p opt hf/Gen geom $=$ connectivity scfcyc $=900$

Input coordinates:

$\begin{array}{lccc}\mathrm{Cl} & 3.87608500 & -5.21315900 & -4.18001000 \\ \mathrm{Mg} & 3.79650900 & -2.82826300 & -3.95881900 \\ \mathrm{Mg} & 3.48708000 & -5.76721900 & -1.89046800 \\ \mathrm{Cl} & 4.26301200 & -3.25946300 & -1.47544900 \\ \mathrm{Cl} & 1.21345800 & -4.91795100 & -1.25514300 \\ \mathrm{Mg} & 2.01804100 & -2.50188200 & -0.78335100 \\ \mathrm{Cl} & 1.55009900 & -2.07247400 & -3.26882600 \\ \mathrm{Cl} & 4.59706600 & -0.40985900 & -3.48383800 \\ \mathrm{Cl} & 4.10251600 & -2.21789500 & -6.31688000 \\ \mathrm{Mg} & 4.01312200 & 0.06998500 & -5.82199600 \\ \mathrm{Cl} & 4.10815800 & -6.02568000 & 0.57156900 \\ \mathrm{Mg} & 3.20727700 & -8.45055000 & 0.51163900 \\ \mathrm{Cl} & 3.04037400 & -8.11621900 & -1.87131600 \\ \mathrm{Mg} & 2.31981600 & 0.43463300 & -2.85619100\end{array}$




$\begin{array}{lccc}\mathrm{Cl} & 1.72225900 & -3.11194400 & 1.57666400 \\ \mathrm{Mg} & 1.80583500 & -5.39970800 & 1.08099400 \\ \mathrm{Mg} & 2.59900100 & 3.11635600 & -5.26054400 \\ \mathrm{Cl} & 1.93099700 & -0.11743400 & -0.56527200 \\ \mathrm{Cl} & 1.70684300 & 0.68919300 & -5.32160700 \\ \mathrm{Cl} & 4.66641000 & 2.24670600 & -6.30166000 \\ \mathrm{Cl} & 1.14768300 & -7.57464600 & 1.56256600 \\ \mathrm{Cl} & 4.37528700 & -10.05467700 & 1.50794900 \\ \mathrm{Cl} & 1.42906700 & 4.71610100 & -6.26167700 \\ \mathrm{Cl} & 2.75697400 & 2.78540900 & -2.87629400\end{array}$

Output coordinates:

$\begin{array}{lccc}\mathrm{Cl} & 1.333540 & -2.972772 & 0.360576 \\ \mathrm{Mg} & -0.622011 & -1.684997 & -0.152114 \\ \mathrm{Mg} & 2.971148 & -1.288124 & 0.030250 \\ \mathrm{Cl} & 0.949918 & -0.149338 & -1.449682 \\ \mathrm{Cl} & 2.531459 & 0.599395 & 1.603684 \\ \mathrm{Mg} & 0.622006 & 1.685362 & 0.150056 \\ \mathrm{Cl} & -0.950168 & 0.149187 & 1.448451 \\ \mathrm{Cl} & -2.532079 & -0.597423 & -1.604742 \\ \mathrm{Cl} & -2.330099 & -3.376067 & 0.342298 \\ \mathrm{Mg} & -4.071662 & -1.946093 & -0.255273 \\ \mathrm{Cl} & 4.405067 & 0.224221 & -1.448674 \\ \mathrm{Mg} & 6.459393 & -0.460281 & -0.018881 \\ \mathrm{Cl} & 5.008108 & -2.116359 & 0.945027 \\ \mathrm{Mg} & -2.970638 & 1.287924 & -0.028413 \\ \mathrm{Cl} & 2.330421 & 3.375560 & -0.347096 \\ \mathrm{Mg} & 4.071583 & 1.946239 & 0.252684 \\ \mathrm{Mg} & -6.459 \mathrm{Cl} 8 & 0.459903 & 0.021190 \\ \mathrm{Cl} & -1.333677 & 2.973441 & -0.359953 \\ \mathrm{Cl} & -4.404631 & -0.226992 & 1.448963 \\ \mathrm{Cl} & -6.193013 & -1.695244 & -1.141387 \\ \mathrm{Cl} & 6.192595 & 1.696700 & 1.139975 \\ \mathrm{Cl} & 8.370630 & -0.921275 & -1.017630 \\ \mathrm{Cl} & -8.370260 & 0.919358 & 1.020993 \\ \mathrm{Cl} & -5.008264 & 2.117654 & -0.940451\end{array}$

\section{$\mathrm{Mg}_{9} \mathrm{Cl}_{18}$ (Optimized)}

Input Keywords:

\#p opt hf/Gen geom=connectivity scfcyc $=800$

Input coordinates:

$\begin{array}{lccc}\mathrm{Cl} & 0.99934400 & -12.83950400 & -2.48846400 \\ \mathrm{Cl} & 4.76936300 & -13.00114600 & -2.40629200 \\ \mathrm{Cl} & -2.64756300 & -11.06766900 & -0.34965800 \\ \mathrm{Mg} & -0.56650400 & -11.95101700 & -0.98195600 \\ \mathrm{Cl} & 1.18368400 & -10.51323400 & -0.08442600 \\ \mathrm{Cl} & -0.62160700 & -13.37151700 & 0.99671200 \\ \mathrm{Mg} & 2.97268400 & -12.06975700 & -1.18590200 \\ \mathrm{Cl} & 4.66476300 & -10.51892200 & -0.06151800 \\ \mathrm{Cl} & 2.95935900 & -13.37231500 & 0.96061900 \\ \mathrm{Mg} & 6.52448300 & -11.87596300 & -1.14954800 \\ \mathrm{Cl} & 6.77949600 & -12.98101000 & 1.00444800 \\ \mathrm{Mg} & -2.45983800 & -11.81524000 & 2.01444500 \\ \mathrm{Cl} & -0.58228300 & -10.49385200 & 3.03074800 \\ \mathrm{Mg} & 1.18848600 & -11.93322600 & 1.99572200 \\ \mathrm{Cl} & 2.99845600 & -10.49472400 & 2.99477400 \\ \mathrm{Cl} & 1.19336600 & -13.35302100 & 4.07583100 \\ \mathrm{Mg} & 4.83687500 & -12.05080500 & 1.97703800 \\ \mathrm{Cl} & 5.02468700 & -12.79833500 & 4.34114400 \\ \mathrm{Mg} & -0.59573100 & -11.79626000 & 5.17740000\end{array}$




$\begin{array}{lccc}\mathrm{Cl} & 1.37763400 & -11.02692700 & 6.48004000 \\ \mathrm{Mg} & 2.94350100 & -11.91524400 & 4.97340200 \\ \mathrm{Cl} & 8.20947800 & -10.81997000 & -2.14885700 \\ \mathrm{Cl} & -2.39240300 & -10.86419900 & 6.39736100 \\ \mathrm{Cl} & -4.40270600 & -10.88542700 & 2.98684300 \\ \mathrm{Cl} & -2.28764500 & -13.34708900 & 4.05327200 \\ \mathrm{Mg} & -4.14746100 & -11.98995600 & 5.14107900 \\ \mathrm{Cl} & -5.83222000 & -13.04608100 & 6.14064300\end{array}$

Output coordinates:

$\begin{array}{lccc}\mathrm{Cl} & -2.120532 & 3.972198 & 0.843403 \\ \mathrm{Cl} & -5.322350 & 1.996565 & 1.017426 \\ \mathrm{Cl} & 2.120513 & 3.972199 & -0.843401 \\ \mathrm{Mg} & -0.000005 & 3.446555 & 0.000013 \\ \mathrm{Cl} & -1.030943 & 1.795866 & -1.454259 \\ \mathrm{Cl} & 1.030960 & 1.795880 & 1.454294 \\ \mathrm{Mg} & -3.161885 & 1.842949 & 0.097575 \\ \mathrm{Cl} & -4.057910 & -0.000049 & -1.463404 \\ \mathrm{Cl} & -2.082055 & 0.000012 & 1.417206 \\ \mathrm{Mg} & -6.178092 & -0.000011 & -0.061698 \\ \mathrm{Cl} & -5.322327 & -1.996503 & 1.0 \mathrm{Cl} 558 \\ \mathrm{Mg} & 3.161867 & 1.842949 & -0.097594 \\ \mathrm{Cl} & 2.082027 & 0.000019 & -1.417191 \\ \mathrm{Mg} & -0.000004 & -0.000001 & 0.000044 \\ \mathrm{Cl} & -1.030909 & -1.795855 & -1.454273 \\ \mathrm{Cl} & 1.030894 & -1.795844 & 1.454298 \\ \mathrm{Mg} & -3.161899 & -1.842959 & 0.097591 \\ \mathrm{Cl} & -2.120546 & -3.972215 & 0.843342 \\ \mathrm{Mg} & 3.161931 & -1.842944 & -0.0976 \mathrm{Cl} \\ \mathrm{Cl} & 2.120568 & -3.972204 & -0.843303 \\ \mathrm{Mg} & 0.000005 & -3.446529 & 0.000016 \\ \mathrm{Cl} & -8.154353 & -0.000049 & -1.053227 \\ \mathrm{Cl} & 5.322327 & -1.996445 & -1.017676 \\ \mathrm{Cl} & 5.322350 & 1.996606 & -1.017381 \\ \mathrm{Cl} & 4.057918 & -0.000106 & 1.463384 \\ \mathrm{Mg} & 6.178101 & -0.000006 & 0.061667 \\ \mathrm{Cl} & 8.154356 & -0.000076 & 1.053207\end{array}$

\section{$\mathrm{Mg}_{9} \mathrm{Cl}_{18}$ (Frozen)}

Input Keywords:

$\#$ p hf/Gen geom=connectivity scfcyc=800

Input coordinates:

$\begin{array}{lccc}\mathrm{Cl} & -17.98247800 & -7.95491400 & -1.36300000 \\ \mathrm{Cl} & -17.98247800 & -4.31391400 & -1.36300000 \\ \mathrm{Cl} & -17.98247800 & -0.67291400 & -1.36300000 \\ \mathrm{Cl} & -15.88047800 & -11.59591400 & 1.36300000 \\ \mathrm{Mg} & -16.93147800 & -9.77491400 & 0.00000000 \\ \mathrm{Cl} & -15.88047800 & -7.95491400 & 1.36300000 \\ \mathrm{Cl} & -14.82947800 & -9.77491400 & -1.36300000 \\ \mathrm{Mg} & -16.93147800 & -6.13391400 & 0.00000000 \\ \mathrm{Cl} & -15.88047800 & -4.31391400 & 1.36300000 \\ \mathrm{Cl} & -14.82947800 & -6.13391400 & -1.36300000 \\ \mathrm{Mg} & -16.93147800 & -2.49291400 & 0.00000000 \\ \mathrm{Cl} & -14.82947800 & -2.49291400 & -1.36300000 \\ \mathrm{Cl} & -12.72747800 & -13.41691400 & 1.36300000 \\ \mathrm{Mg} & -13.77847800 & -11.59591400 & 0.00000000 \\ \mathrm{Cl} & -12.72747800 & -9.77491400 & 1.36300000 \\ \mathrm{Cl} & -11.67547800 & -11.59591400 & -1.36300000 \\ \mathrm{Mg} & -13.77847800 & -7.95491400 & 0.00000000 \\ \mathrm{Cl} & -12.72747800 & -6.13391400 & 1.36300000\end{array}$




$\begin{array}{llll}\mathrm{Cl} & -11.67547800 & -7.95491400 & -1.36300000 \\ \mathrm{Mg} & -13.77847800 & -4.31391400 & 0.00000000 \\ \mathrm{Cl} & -11.67547800 & -4.31391400 & -1.36300000 \\ \mathrm{Cl} & -9.57347800 & -15.23691400 & 1.36300000 \\ \mathrm{Mg} & -10.62447800 & -13.41691400 & 0.00000000 \\ \mathrm{Cl} & -9.57347800 & -11.59591400 & 1.36300000 \\ \mathrm{Mg} & -10.62447800 & -9.77491400 & 0.00000000 \\ \mathrm{Cl} & -9.57347800 & -7.95491400 & 1.36300000 \\ \mathrm{Mg} & -10.62447800 & -6.13391400 & 0.00000000\end{array}$

\section{$\mathrm{Mg}_{10} \mathrm{Cl}_{20}$ (Optimized)}

Input Keywords:

\#p opt hf/Gen geom=connectivity scfcyc $=800$

Input coordinates:

$\begin{array}{lccc}\mathrm{Cl} & 4.31871400 & -0.55454100 & -2.62560900 \\ \mathrm{Mg} & 4.19570200 & 1.81882900 & -2.52351300 \\ \mathrm{Mg} & 4.05186200 & -0.97896600 & -0.27499900 \\ \mathrm{Cl} & 4.71782500 & 1.51288500 & -0.00880300 \\ \mathrm{Cl} & 1.75108000 & -0.29298300 & 0.37834200 \\ \mathrm{Mg} & 2.41683800 & 2.19852300 & 0.64398100 \\ \mathrm{Cl} & 1.92224300 & 2.47919000 & -1.85107500 \\ \mathrm{Cl} & 4.88391700 & 4.28545500 & -2.17174600 \\ \mathrm{Cl} & 4.49364800 & 2.31991300 & -4.91225700 \\ \mathrm{Mg} & 4.28036300 & 4.61835300 & -4.53133500 \\ \mathrm{Cl} & 4.54657500 & -1.26025000 & 2.21947700 \\ \mathrm{Mg} & 3.89849500 & -3.83383000 & 1.95178900 \\ \mathrm{Cl} & 4.23485400 & -3.38211600 & -0.35478900 \\ \mathrm{Mg} & 2.56727600 & 5.05187900 & -1.58418600 \\ \mathrm{Cl} & 2.15040300 & 1.77474100 & 2.99489100 \\ \mathrm{Mg} & 2.27434700 & -0.59844100 & 2.89331600 \\ \mathrm{Mg} & 2.69823000 & 7.60566200 & -4.13960900 \\ \mathrm{Cl} & 2.23253400 & 4.60123700 & 0.72308200 \\ \mathrm{Cl} & 1.94289100 & 5.13243100 & -4.05915200 \\ \mathrm{Cl} & 4.81393600 & 6.79737700 & -5.13195200 \\ \mathrm{Cl} & 1.58380200 & -3.06417900 & 2.54270800 \\ \mathrm{Mg} & 2.19088700 & -3.39794100 & 4.90115700 \\ \mathrm{Cl} & 3.57926300 & -6.20066000 & 2.11097500 \\ \mathrm{Cl} & 4.52702200 & -3.91611200 & 4.42470800 \\ \mathrm{Mg} & 3.76696900 & -6.38799300 & 4.50599700 \\ \mathrm{Cl} & 1.44162300 & 9.07527600 & -5.23199700 \\ \mathrm{Cl} & 2.88078800 & 7.41932700 & -1.74416200 \\ \mathrm{Cl} & 1.98183900 & -1.09939300 & 5.28279900 \\ \mathrm{Cl} & 5.02303800 & -7.86034500 & 5.59525100 \\ \mathrm{Cl} & 1.65513400 & -5.57593900 & 5.50337700\end{array}$

Output coordinates:

$\begin{array}{lccc}\mathrm{Cl} & -0.608157 & -3.155993 & 0.236853 \\ \mathrm{Mg} & -2.505426 & -1.795092 & -0.188047 \\ \mathrm{Mg} & 1.075766 & -1.497314 & -0.185148 \\ \mathrm{Cl} & -0.853532 & -0.292296 & -1.512715 \\ \mathrm{Cl} & 0.853354 & 0.292926 & 1.512011 \\ \mathrm{Mg} & -1.075723 & 1.497437 & 0.184984 \\ \mathrm{Cl} & -2.694342 & 0.011152 & 1.445166 \\ \mathrm{Cl} & -4.366039 & -0.621993 & -1.605057 \\ \mathrm{Cl} & -4.288100 & -3.409935 & 0.339413 \\ \mathrm{Mg} & -5.957010 & -1.895504 & -0.234632 \\ \mathrm{Cl} & 2.694345 & -0.012113 & -1.445717 \\ \mathrm{Mg} & 4.721356 & -1.289701 & 0.038233 \\ \mathrm{Cl} & 3.003836 & -2.870696 & 0.328966 \\ \mathrm{Mg} & -4.721125 & 1.289538 & -0.036426\end{array}$




$\begin{array}{lccc}\mathrm{Cl} & 0.608140 & 3.156018 & -0.238594 \\ \mathrm{Mg} & 2.505413 & 1.795304 & 0.186106 \\ \mathrm{Mg} & -8.234528 & 0.615000 & 0.053409 \\ \mathrm{Cl} & -3.003978 & 2.871217 & -0.326695 \\ \mathrm{Cl} & -6.185984 & -0.148535 & 1.461466 \\ \mathrm{Cl} & -8.076441 & -1.555967 & -1.095194 \\ \mathrm{Cl} & 4.365726 & 0.623672 & 1.604447 \\ \mathrm{Mg} & 5.956984 & 1.895621 & 0.232811 \\ \mathrm{Cl} & 6.728759 & -2.193388 & 0.951306 \\ \mathrm{Cl} & 6.186236 & 0.146501 & -1.461090 \\ \mathrm{Mg} & 8.234633 & -0.615259 & -0.051700 \\ \mathrm{Cl} & -10.105523 & 1.158107 & 1.086059 \\ \mathrm{Cl} & -6.728804 & 2.194356 & -0.947880 \\ \mathrm{Cl} & 4.288241 & 3.409437 & -0.343202 \\ \mathrm{Cl} & 10.105770 & -1.159595 & -1.083426 \\ \mathrm{Cl} & 8.076251 & 1.557105 & 1.094171\end{array}$

\section{$\mathrm{Mg}_{12} \mathrm{Cl}_{24}($ Optimized $)$}

Input Keywords:

\#p opt hf/Gen geom=connectivity scfcyc $=800$

Input coordinates:

$\begin{array}{lccc}\mathrm{Cl} & 2.67470500 & -12.68479900 & -5.56049900 \\ \mathrm{Cl} & -0.8947600 & -10.75673800 & -3.49273800 \\ \mathrm{Mg} & 1.17238300 & -11.74207100 & -4.03356900 \\ \mathrm{Cl} & 2.98117200 & -10.39044400 & -3.11849700 \\ \mathrm{Cl} & 1.00287900 & -13.14454100 & -2.05363700 \\ \mathrm{Mg} & 4.68476900 & -12.03178000 & -4.24767800 \\ \mathrm{Cl} & 4.56573900 & -13.37207900 & -2.14005800 \\ \mathrm{Cl} & -2.74946400 & -10.48166300 & -0.20298700 \\ \mathrm{Mg} & -0.79099100 & -11.47459100 & -1.13734400 \\ \mathrm{Cl} & 1.14531400 & -10.32500800 & -0.07422900 \\ \mathrm{Cl} & -0.87078400 & -12.96598700 & 0.92393000 \\ \mathrm{Mg} & 2.94504200 & -11.80214800 & -1.03394500 \\ \mathrm{Cl} & 4.85150600 & -10.60907600 & 0.03943500 \\ \mathrm{Cl} & 2.83451300 & -13.24907700 & 1.03716300 \\ \mathrm{Mg} & 6.55427600 & -12.22490600 & -1.10013700 \\ \mathrm{Cl} & 6.72685300 & -13.09736300 & 1.16525000 \\ \mathrm{Cl} & -0.58656800 & -10.20347000 & 3.10361000 \\ \mathrm{Mg} & 1.03498000 & -11.77162300 & 1.99682300 \\ \mathrm{Cl} & 2.97673000 & -10.42972300 & 3.01645200 \\ \mathrm{Cl} & 0.99953900 & -13.18438500 & 4.08108500 \\ \mathrm{Mg} & 4.77053300 & -12.10064000 & 2.10041700 \\ \mathrm{Cl} & 4.87488000 & -12.81639300 & 4.45610000 \\ \mathrm{Mg} & 2.80672300 & -11.83175000 & 4.99641700 \\ \mathrm{Cl} & 8.42890600 & -13.21706700 & -2.16517400 \\ \mathrm{Cl} & 6.41564200 & -12.99232900 & -5.53514600 \\ \mathrm{Cl} & -4.44834200 & -10.35795400 & 3.12881000 \\ \mathrm{Mg} & -2.57476500 & -11.35162600 & 2.06321700 \\ \mathrm{Cl} & -2.46477400 & -12.97397500 & 4.04912400 \\ \mathrm{Mg} & -4.24766900 & -11.57882900 & 5.21301100 \\ \mathrm{Cl} & -2.4348300 & -10.58448900 & 6.49917500 \\ \mathrm{Mg} & -0.70504700 & -11.54576600 & 5.21055300 \\ \mathrm{Cl} & 1.30432900 & -10.89158200 & 6.52453800 \\ \mathrm{Cl} & -5.96572800 & -12.60444600 & 6.19116400 \\ \mathrm{Mg} & 8.22795500 & -11.99616000 & -4.24971800 \\ \mathrm{Cl} & 6.44403300 & -10.60251700 & -3.08616000 \\ \mathrm{Cl} & 9.94516800 & -10.97072300 & -5.22924900 \\ \mathrm{O} & & & \end{array}$

Output coordinates:

$\begin{array}{lllll}\mathrm{Cl} & 4.591141 & 3.980320 & -0.951625\end{array}$ 


$\begin{array}{lccc}\mathrm{Cl} & 0.464570 & 4.946416 & 0.658091 \\ \mathrm{Mg} & 2.406852 & 3.887700 & -0.130374 \\ \mathrm{Cl} & 3.072653 & 2.125449 & 1.401662 \\ \mathrm{Cl} & 1.047830 & 2.446807 & -1.526629 \\ \mathrm{Mg} & 5.189246 & 1.712250 & -0.130521 \\ \mathrm{Cl} & 3.764411 & 0.107247 & -1.420131 \\ \mathrm{Cl} & -3.134291 & 3.776907 & 0.834994 \\ \mathrm{Mg} & -1.009560 & 3.086035 & 0.010453 \\ \mathrm{Cl} & -0.333396 & 1.106010 & 1.360300 \\ \mathrm{Cl} & -2.370144 & 1.481896 & -1.436397 \\ \mathrm{Mg} & 1.731712 & 0.482867 & 0.024122 \\ \mathrm{Cl} & 2.370307 & -1.482230 & 1.436411 \\ \mathrm{Cl} & 0.333346 & -1.105962 & -1.360096 \\ \mathrm{Mg} & 4.479167 & -1.898765 & -0.075344 \\ \mathrm{Cl} & 3.134223 & -3.776685 & -0.835708 \\ \mathrm{Cl} & -3.764572 & -0.107139 & 1.420152 \\ \mathrm{Mg} & -1.731794 & -0.482971 & -0.023770 \\ \mathrm{Cl} & -1.047878 & -2.446620 & 1.526808 \\ \mathrm{Cl} & -3.072733 & -2.125696 & -1.401431 \\ \mathrm{Mg} & 1.009663 & -3.086076 & -0.010588 \\ \mathrm{Cl} & -0.464555 & -4.946519 & -0.657554 \\ \mathrm{Mg} & -2.406897 & -3.887771 & 0.130842 \\ \mathrm{Cl} & 6.610847 & -2.438058 & -0.944087 \\ \mathrm{Cl} & 7.360685 & 1.456600 & -0.996777 \\ \mathrm{Cl} & -6.610823 & 2.437923 & 0.944094 \\ \mathrm{Mg} & -4.479178 & 1.898795 & 0.075066 \\ \mathrm{Cl} & -5.681322 & 0.214618 & -1.484500 \\ \mathrm{Mg} & -7.797893 & 0.639099 & -0.144769 \\ \mathrm{Cl} & -7.360704 & -1.456693 & 0.996355 \\ \mathrm{Mg} & -5.189150 & -1.712240 & 0.130340 \\ \mathrm{Cl} & -4.591153 & -3.980 \mathrm{Cl} & 0.952142 \\ \mathrm{Cl} & -9.714370 & 0.976365 & -1.197756 \\ \mathrm{Mg} & 7.797895 & -0.639061 & 0.144561 \\ \mathrm{Cl} & 5.681398 & -0.214467 & 1.484292 \\ \mathrm{Cl} & 9.714484 & -0.976215 & 1.197380\end{array}$

\section{$\mathrm{Mg}_{15} \mathrm{Cl}_{30}$ (Optimized)}

Input Keywords:

\#p opt hf/Gen geom=connectivity scfcyc $=800$

Input coordinates:

$\begin{array}{llll}\mathrm{Mg} & 3.61265600 & -5.47589500 & -1.21093300\end{array}$

$\mathrm{Cl} \quad 4.06415900 \quad-3.23901200 \quad-1.76912600$

$\mathrm{Cl} \quad 1.26920800 \quad-4.89881600 \quad-0.87711300$

$\begin{array}{llll}\mathrm{Mg} & 1.99797900 & -2.41754400 & -0.69373400\end{array}$

$\begin{array}{llll}\mathrm{Cl} & 4.03664900 & -5.80487000 & 1.16182100\end{array}$

$\begin{array}{llll}\mathrm{Mg} & 3.49245000 & -8.34158200 & 0.83275000\end{array}$

$\begin{array}{llll}\mathrm{Cl} & 3.84764900 & -7.77472100 & -1.56532500\end{array}$

$\begin{array}{llll}\mathrm{Cl} & 2.17735800 & -2.84333800 & 1.75461800\end{array}$

$\begin{array}{llll}\mathrm{Mg} & 1.61009100 & -5.30113500 & 1.64627200\end{array}$

$\begin{array}{llll}\mathrm{Mg} & -0.24402500 & -2.27748400 & 2.22078600\end{array}$

$\mathrm{Cl} \quad 2.25359100 \quad-0.06657200 \quad-1.04486100$

$\mathrm{Cl} \quad-0.45309000 \quad-1.85298500 \quad-0.27351400$

$\begin{array}{llll}\mathrm{Mg} & 0.18510400 & 0.66117100 & -0.03394400\end{array}$

$\mathrm{Cl} \quad 1.11234600 \quad-7.73865000 \quad 1.29519000$

$\begin{array}{llll}\mathrm{Mg} & 1.30233200 & -8.23472300 & 3.76184600\end{array}$

$\begin{array}{lllll}\mathrm{Cl} & 3.58639500 & -10.69979700 & 0.76155900\end{array}$

$\mathrm{Cl} \quad 3.82373600 \quad-8.47573800 \quad 3.35299600$

$\begin{array}{llll}\mathrm{Mg} & 3.50187000 & -10.99460400 & 3.17739400\end{array}$

$\begin{array}{llll}\mathrm{Mg} & -1.78575300 & 3.68355100 & 0.68365300\end{array}$ 


$\begin{array}{lccc}\mathrm{Cl} & -0.81044400 & -4.72774200 & 2.03059100 \\ \mathrm{Cl} & -0.03508900 & -2.69975700 & 4.71517300 \\ \mathrm{Mg} & -2.10131700 & 0.74318200 & 2.79224700 \\ \mathrm{Mg} & -0.67057400 & -5.21288900 & 4.47785800 \\ \mathrm{Mg} & -2.48706700 & -2.13605500 & 5.13623900 \\ \mathrm{Cl} & 0.34260800 & 3.04171500 & -0.26816400 \\ \mathrm{Cl} & 0.32093500 & 0.17271000 & 2.41294100 \\ \mathrm{Cl} & -2.29640100 & 1.13997000 & 0.34801700 \\ \mathrm{Cl} & -2.66642800 & -1.71299900 & 2.68684100 \\ \mathrm{Cl} & 1.81059000 & -5.69020500 & 4.09188600 \\ \mathrm{Cl} & -0.82590500 & -7.59344000 & 4.71356100 \\ \mathrm{Cl} & 4.91035500 & -12.35012800 & 4.24578000 \\ \mathrm{Cl} & 1.22292100 & -10.60684300 & 3.89509100 \\ \mathrm{Cl} & -2.73993300 & -4.48699400 & 5.48848500 \\ \mathrm{Cl} & -1.70555000 & 6.05558800 & 0.55190800 \\ \mathrm{Cl} & -1.60069400 & 3.18179800 & 3.14896300 \\ \mathrm{Cl} & -4.30890300 & 3.92510600 & 1.08858800 \\ \mathrm{Mg} & -3.98545200 & 6.44262300 & 1.26777800 \\ \mathrm{Mg} & -3.97971500 & 3.78760800 & 3.60957200 \\ \mathrm{Cl} & -1.76076900 & 0.34436800 & 5.31826500 \\ \mathrm{Cl} & -4.52629600 & 1.25073800 & 3.27662600 \\ \mathrm{Cl} & -4.55653900 & -1.31643600 & 6.20588200 \\ \mathrm{Mg} & -4.10458000 & 0.92040600 & 5.64943700 \\ \mathrm{Cl} & -4.07116100 & 6.14556100 & 3.68379200 \\ \mathrm{Cl} & -4.34075700 & 3.21859800 & 6.00603000 \\ \mathrm{Cl} & -5.39014800 & 7.80201700 & 0.19927100 \\ \mathrm{Ou} & \mathrm{C} & & \end{array}$

Output coordinates:

$\begin{array}{lccc}\mathrm{Mg} & 4.612380 & -3.931664 & 0.191257 \\ \mathrm{Cl} & 2.802920 & -5.209255 & -0.573788 \\ \mathrm{Cl} & 3.097729 & -2.637247 & 1.578443 \\ \mathrm{Mg} & 1.140158 & -3.503719 & 0.073832 \\ \mathrm{Cl} & 5.060041 & -2.130143 & -1.368406 \\ \mathrm{Mg} & 7.129848 & -1.465818 & 0.134151 \\ \mathrm{Cl} & 6.796089 & -3.774126 & 0.996273 \\ \mathrm{Cl} & 1.564029 & -1.486822 & -1.328255 \\ \mathrm{Mg} & 3.550387 & -0.618475 & -0.001569 \\ \mathrm{Mg} & 0.000004 & 0.000009 & 0.000021 \\ \mathrm{Cl} & -0.865318 & -4.541229 & -0.701493 \\ \mathrm{Cl} & -0.423481 & -2.048143 & 1.475570 \\ \mathrm{Mg} & -2.436216 & -2.845079 & -0.016707 \\ \mathrm{Cl} & 5.546414 & -0.018662 & 1.422197 \\ \mathrm{Mg} & 6.030941 & 2.038085 & 0.048 \mathrm{Mg} 5 \\ \mathrm{Cl} & 9.272279 & -0.967640 & 0.965192 \\ \mathrm{Cl} & 7.390703 & 0.477880 & -1.511864 \\ \mathrm{Mg} & 9.467978 & 1.146081 & -0.210515 \\ \mathrm{Mg} & -6.031001 & -2.038105 & -0.048672 \\ \mathrm{Cl} & 1.976933 & 0.797370 & 1.372362 \\ \mathrm{Cl} & 0.423507 & 2.048116 & -1.475391 \\ \mathrm{Mg} & -3.550486 & 0.618474 & 0.002363 \\ \mathrm{Mg} & 2.436187 & 2.845135 & 0.016588 \\ \mathrm{Mg} & -1.140275 & 3.503733 & -0.073454 \\ \mathrm{Cl} & -4.505859 & -3.745983 & -0.816225 \\ \mathrm{Cl} & -1.976910 & -0.797209 & -1.372102 \\ \mathrm{Cl} & -3.953648 & -1.378175 & 1.438619 \\ \mathrm{Cl} & -1.563947 & 1.486727 & 1.328598 \\ \mathrm{Cl} & 3.953517 & 1.377833 & -1.438548 \\ \mathrm{Cl} & 4.505938 & 3.746244 & 0.815508 \\ \mathrm{Cl} & 11.322318 & 1.667719 & -1.300098 \\ \mathrm{Cl} & 8.107668 & 2.812482 & 0.879727 \\ \mathrm{Cl} & 0.865262 & 4.541196 & 0.701660 \\ \mathrm{Cl} & -8.107642 & -2.812458 & -0.880505\end{array}$




$\begin{array}{lccc}\mathrm{Cl} & -5.546131 & 0.018861 & -1.422220 \\ \mathrm{Cl} & -7.390874 & -0.478005 & 1.511348 \\ \mathrm{Mg} & -9.468088 & -1.146166 & 0.209681 \\ \mathrm{Mg} & -7.129927 & 1.465715 & -0.134387 \\ \mathrm{Cl} & -3.097630 & 2.637611 & -1.577936 \\ \mathrm{Cl} & -5.059985 & 2.129900 & 1.368819 \\ \mathrm{Cl} & -2.802960 & 5.209200 & 0.574796 \\ \mathrm{Mg} & -4.612331 & 3.93 \mathrm{Cl} 32 & -0.190551 \\ \mathrm{Cl} & -9.272 \mathrm{Cl} 3 & 0.967570 & -0.965929 \\ \mathrm{Cl} & -6.796021 & 3.774161 & -0.995702 \\ \mathrm{Cl} & -11.322454 & -1.667731 & 1.299240\end{array}$

\section{$\mathrm{Mg}_{16} \mathrm{Cl}_{32}$ (Optimized)}

Input Keywords:

\#p Opt hf/Gen geom=connectivity scfcyc=800

Input coordinates:

\begin{tabular}{lccc}
$\mathrm{Cl}$ & -0.95067100 & -12.67149000 & -5.62423400 \\
$\mathrm{Cl}$ & 2.82993900 & -12.85379600 & -5.64914800 \\
$\mathrm{Mg}$ & -2.47409500 & -11.75514100 & -4.08670100 \\
$\mathrm{Cl}$ & -0.68229500 & -10.37633800 & -3.18373300 \\
$\mathrm{Cl}$ & -2.60405100 & -13.19364300 & -2.12683800 \\
$\mathrm{Mg}$ & 1.05041200 & -11.99154600 & -4.32902900 \\
$\mathrm{Cl}$ & 2.86013800 & -10.53584000 & -3.27188900 \\
$\mathrm{Cl}$ & 1.01006000 & -13.25283700 & -2.19598600 \\
$\mathrm{Mg}$ & 4.70347800 & -12.04856900 & -4.30863200 \\
$\mathrm{Cl}$ & 4.6353490 & -13.37384500 & -2.17064000 \\
$\mathrm{Cl}$ & -2.53761200 & -10.37946600 & -0.07827100 \\
$\mathrm{Mg}$ & -0.74208800 & -11.81554400 & -1.09869400 \\
$\mathrm{Cl}$ & 1.11067700 & -10.49723600 & -0.06626700 \\
$\mathrm{Cl}$ & -0.78512700 & -13.20100500 & 0.97886700 \\
$\mathrm{Mg}$ & 2.92363200 & -11.85707100 & -1.14668000 \\
$\mathrm{Cl}$ & 4.75964700 & -10.60153100 & 0.00159900 \\
$\mathrm{Cl}$ & 2.86235400 & -13.31794700 & 0.99215100 \\
$\mathrm{Mg}$ & 6.54803300 & -12.11235200 & -1.13024500 \\
$\mathrm{Cl}$ & 6.76903100 & -12.98873200 & 1.13649700 \\
$\mathrm{Cl}$ & -0.68601700 & -10.44540300 & 3.10900800 \\
$\mathrm{Mg}$ & 1.06587400 & -11.88145300 & 2.01206300 \\
$\mathrm{Cl}$ & 2.92817200 & -10.50273900 & 3.03935400 \\
$\mathrm{Cl}$ & 1.00907100 & -13.32117800 & 4.09703700 \\
$\mathrm{Mg}$ & 4.75498700 & -12.11925000 & 2.05320000 \\
$\mathrm{Cl}$ & 4.87462000 & -12.87462100 & 4.41086800 \\
$\mathrm{Mg}$ & 2.79964900 & -11.94064500 & 4.99970900 \\
$\mathrm{Cl}$ & 8.51425500 & -12.95928000 & -2.15095700 \\
$\mathrm{Cl}$ & 6.55041900 & -12.89112500 & -5.53509200 \\
$\mathrm{Cl}$ & -4.54840700 & -10.81982800 & -3.49750900 \\
$\mathrm{Cl}$ & -6.44452500 & -10.70878200 & -0.22340300 \\
$\mathrm{Mg}$ & -4.43028300 & -11.57728100 & -1.14039100 \\
$\mathrm{Cl}$ & -4.43481100 & -13.09663600 & 0.91008800 \\
$\mathrm{Mg}$ & -6.22357600 & -11.58604000 & 2.04301800 \\
$\mathrm{Cl}$ & -4.31072700 & -10.32601600 & 3.08415900 \\
$\mathrm{Mg}$ & -2.59926800 & -11.84255300 & 2.05957800 \\
$\mathrm{Cl}$ & -2.53465100 & -13.16370400 & 4.18492500 \\
$\mathrm{Mg}$ & -4.37858900 & -11.65267300 & 5.22176100 \\
$\mathrm{Cl}$ & -2.50535000 & -10.84592600 & 6.56195800 \\
$\mathrm{Mg}$ & -0.72503900 & -11.70670800 & 5.24201300 \\
$\mathrm{Cl}$ & 1.27543900 & -11.02551000 & 6.53713900 \\
$\mathrm{Cl}$ & -6.22568000 & -10.81272300 & 6.44950300 \\
$\mathrm{Cl}$ & -8.18919000 & -10.73980200 & 3.06511500 \\
$\mathrm{Mg}$ & 8.25148600 & -11.74989000 & -4.23667700 \\
& \multicolumn{2}{c}{-1}
\end{tabular}




\begin{tabular}{|c|c|c|c|}
\hline $\mathrm{Cl}$ & 6.34979300 & -10.49726600 & -3.10784000 \\
\hline $\mathrm{Cl}$ & 9.91077400 & -10.59540100 & -5.17610000 \\
\hline $\mathrm{Mg}$ & -7.92621500 & -11.95236100 & 0 5.14920500 \\
\hline $\mathrm{Cl}$ & -6.02467500 & -13.20312800 & 4.01875400 \\
\hline $\mathrm{Cl}$ & -9.58550300 & -13.10839600 & 6.08676000 \\
\hline \multicolumn{4}{|c|}{ Output coordinates: } \\
\hline $\mathrm{Cl}$ & -2.11622700 & -5.81443300 & -0.84044600 \\
\hline $\mathrm{Cl}$ & -5.41006100 & -3.94397700 & -0.91506700 \\
\hline $\mathrm{Mg}$ & -0.00003100 & -5.24800600 & 0.00009400 \\
\hline $\mathrm{Cl}$ & -1.04618200 & -3.61550900 & 1.45961600 \\
\hline $\mathrm{Cl}$ & 1.04609300 & -3.61555800 & -1.45954300 \\
\hline $\mathrm{Mg}$ & -3.19097100 & -3.70477700 & -0.11283000 \\
\hline $\mathrm{Cl}$ & -4.19135800 & -1.89491700 & 1.40372600 \\
\hline $\mathrm{Cl}$ & -2.11875400 & -1.86906300 & -1.38521300 \\
\hline $\mathrm{Mg}$ & -6.34028300 & -1.84620100 & -0.08804400 \\
\hline $\mathrm{Cl}$ & -5.24358600 & -0.00004900 & -1.40506300 \\
\hline $\mathrm{Cl}$ & 2.11897300 & -1.86929200 & 1.38528400 \\
\hline $\mathrm{Mg}$ & 0.00014800 & -1.80709600 & 0.00009000 \\
\hline $\mathrm{Cl}$ & -1.05450400 & -0.00002200 & 1.37728200 \\
\hline $\mathrm{Cl}$ & 1.05442600 & 0.00008700 & -1.37704900 \\
\hline $\mathrm{Mg}$ & -3.21278100 & -0.00009000 & 0.07117900 \\
\hline $\mathrm{Cl}$ & -4.19129000 & 1.89479800 & 1.40369900 \\
\hline $\mathrm{Cl}$ & -2.11882900 & 1.86918800 & -1.38521100 \\
\hline $\mathrm{Mg}$ & -6.34031800 & 1.84616500 & -0.08802500 \\
\hline $\mathrm{Cl}$ & -5.41024700 & 3.94412200 & 69900 \\
\hline $\mathrm{Cl}$ & 2.11882200 & 1.86913900 & 31900 \\
\hline $\mathrm{Mg}$ & -0.00003100 & 1.80714500 & 0.00020800 \\
\hline $\mathrm{Cl}$ & -1.04612900 & 3.61561200 & 1.45958800 \\
\hline $\mathrm{Cl}$ & 1.04624800 & 3.61548200 & -1.45953200 \\
\hline $\mathrm{Mg}$ & -3.19103400 & 3.70473500 & -0.11281800 \\
\hline $\mathrm{Cl}$ & -2.11624800 & 5.81435000 & -0.84064900 \\
\hline $\mathrm{Mg}$ & -0.00003900 & 5.24801700 & -0.00013800 \\
\hline $\mathrm{Cl}$ & -8.54846500 & 1.97199500 & -0.91900500 \\
\hline $\mathrm{Cl}$ & -8.54850700 & -1.97223200 & 74800 \\
\hline $\mathrm{Cl}$ & 2.11620100 & -5.81445100 & 0.84054200 \\
\hline $\mathrm{Cl}$ & 5.41025400 & -3.94413600 & 0.91459200 \\
\hline $\mathrm{Mg}$ & 3.19095500 & -3.70482800 & 0.11283000 \\
\hline $\mathrm{Cl}$ & 4.19122100 & -1.89478400 & -1.40375200 \\
\hline $\mathrm{Mg}$ & 6.34041000 & -1.84618500 & 0.08819300 \\
\hline $\mathrm{Cl}$ & 5.24352100 & 0.00014100 & 1.40507600 \\
\hline $\mathrm{Mg}$ & 3.21295100 & 0.00007600 & -0.07142900 \\
\hline $\mathrm{Cl}$ & 4.19149600 & 1.89491600 & -1.40379800 \\
\hline $\mathrm{Mg}$ & 6.34018900 & 1.84624100 & 0.08789300 \\
\hline $\mathrm{Cl}$ & 5.40997600 & 3.94395400 & 0.91514700 \\
\hline $\mathrm{Mg}$ & 3.19087300 & 3.70487500 & 0.11303100 \\
\hline $\mathrm{Cl}$ & 2.11611000 & 5.81457000 & 0.84045100 \\
\hline $\mathrm{Cl}$ & 8.54854600 & 1.97248800 & 0.91818400 \\
\hline $\mathrm{Cl}$ & 8.54851400 & -1.97184500 & 0.91926400 \\
\hline $\mathrm{Mg}$ & -9.34924300 & -0.00003100 & 0.22441400 \\
\hline $\mathrm{Cl}$ & -7.16701600 & 0.00003700 & 1.51610900 \\
\hline $\mathrm{Cl}$ & -11.27130200 & 0.00001500 & 1.32345700 \\
\hline $\mathrm{Mg}$ & 9.34921500 & 0.00001600 & -0.22455400 \\
\hline $\mathrm{Cl}$ & 7.16697100 & -0.00034400 & -1.51611800 \\
\hline $\mathrm{Cl}$ & 11.27132700 & -0.00032300 & -1.32350700 \\
\hline
\end{tabular}

\section{$\mathrm{Mg}_{16} \mathrm{Cl}_{32}$ (Frozen)}

Input Keywords:

$\#$ phf/Gen geom=connectivity scfcyc $=800$

Input coordinates: 


\begin{tabular}{|c|c|c|}
\hline $\mathrm{Cl}$ & $\begin{array}{ll}-17.98247800 & -7.95491400\end{array}$ & -1.36300000 \\
\hline $\mathrm{Cl}$ & $-17.98247800 \quad-4.31391400$ & -1.36300000 \\
\hline $\mathrm{Cl}$ & $-17.98247800 \quad-0.67291400$ & -1.36300000 \\
\hline $\mathrm{Cl}$ & -17.98247800 & -1.36300000 \\
\hline $\mathrm{Cl}$ & $-15.88047800-11.59591400$ & 1.36300000 \\
\hline $\mathrm{Mg}$ & $-16.93147800 \quad-9.77491400$ & 0.00000000 \\
\hline $\mathrm{Cl}$ & $-15.88047800 \quad-7.95491400$ & 1.36300000 \\
\hline $\mathrm{Cl}$ & $-14.82947800 \quad-9.77491400$ & -1.36300000 \\
\hline $\mathrm{Mg}$ & $-16.93147800-6.13391400$ & 0.00000000 \\
\hline $\mathrm{Cl}$ & $-15.88047800 \quad-4.31391400$ & 1.36300000 \\
\hline $\mathrm{Cl}$ & $-14.82947800-6.13391400$ & -1.36300000 \\
\hline $\mathrm{Mg}$ & $-16.93147800 \quad-2.49291400$ & 0.00000000 \\
\hline $\mathrm{Cl}$ & $-15.88047800-0.67291400$ & 1.36300000 \\
\hline $\mathrm{Cl}$ & $-14.82947800 \quad-2.49291400$ & -1.36300000 \\
\hline $\mathrm{Mg}$ & $-16.93147800 \quad 1.14808600$ & 0.00000000 \\
\hline $\mathrm{Cl}$ & $-14.82947800 \quad 1.14808600$ & -1.36300000 \\
\hline $\mathrm{Cl}$ & $-12.72747800-13.41691400$ & 1.36300000 \\
\hline $\mathrm{Mg}$ & $-13.77847800-11.59591400$ & 0.00000000 \\
\hline $\mathrm{Cl}$ & $-12.72747800 \quad-9.77491400$ & 1.36300000 \\
\hline $\mathrm{Cl}$ & $-11.67547800-11.59591400$ & -1.36300000 \\
\hline $\mathrm{Mg}$ & $-13.77847800 \quad-7.95491400$ & 0.00000000 \\
\hline $\mathrm{Cl}$ & $-12.72747800 \quad-6.13391400$ & 1.36300000 \\
\hline $\mathrm{Cl}$ & $-11.67547800 \quad-7.95491400$ & -1.36300000 \\
\hline $\mathrm{Mg}$ & $-13.77847800 \quad-4.31391400$ & 0.00000000 \\
\hline $\mathrm{Cl}$ & $-12.72747800 \quad-2.49291400$ & 1.36300000 \\
\hline $\mathrm{Cl}$ & $-11.67547800 \quad-4.31391400$ & -1.36300000 \\
\hline $\mathrm{Mg}$ & $-13.77847800 \quad-0.67291400$ & 0.00000000 \\
\hline $\mathrm{Cl}$ & $-11.67547800 \quad-0.67291400$ & -1.36300000 \\
\hline $\mathrm{Cl}$ & $-9.57347800 \quad-15.23691400$ & 1.36300000 \\
\hline $\mathrm{Mg}$ & $-10.62447800-13.41691400$ & 0.00000000 \\
\hline $\mathrm{Cl}$ & $-9.57347800-11.59591400$ & 1.36300000 \\
\hline $\mathrm{Cl}$ & $-8.52247800-13.41691400$ & -1.36300000 \\
\hline $\mathrm{Mg}$ & $-10.62447800 \quad-9.77491400$ & 0.00000000 \\
\hline $\mathrm{Cl}$ & $-9.57347800 \quad-7.95491400$ & 1.36300000 \\
\hline $\mathrm{Cl}$ & $\begin{array}{ll}-8.52247800 & -9.77491400\end{array}$ & -1.36300000 \\
\hline $\mathrm{Mg}$ & $-10.62447800 \quad-6.13391400$ & 0.00000000 \\
\hline $\mathrm{Cl}$ & $-9.57347800 \quad-4.31391400$ & 1.36300000 \\
\hline $\mathrm{Cl}$ & $-8.52247800-6.13391400$ & -1.36300000 \\
\hline $\mathrm{Mg}$ & $-10.62447800 \quad-2.49291400$ & 0.00000000 \\
\hline $\mathrm{Cl}$ & $-8.52247800 \quad-2.49291400$ & -1.36300000 \\
\hline $\mathrm{Cl}$ & $-6.42047800-17.05791400$ & 1.36300000 \\
\hline $\mathrm{Mg}$ & $-7.47147800-15.23691400$ & 0.00000000 \\
\hline $\mathrm{Cl}$ & $-6.42047800-13.41691400$ & 1.36300000 \\
\hline $\mathrm{Mg}$ & $-7.47147800-11.59591400$ & 0.00000000 \\
\hline $\mathrm{Cl}$ & $-6.42047800 \quad-9.77491400$ & 1.36300000 \\
\hline $\mathrm{Mg}$ & $-7.47147800 \quad-7.95491400$ & 0.00000000 \\
\hline $\mathrm{Cl}$ & $-6.42047800 \quad-6.13391400$ & 1.36300000 \\
\hline $\mathrm{Mg}$ & $-7.47147800 \quad-4.31391400$ & 0.00000000 \\
\hline
\end{tabular}

\section{$\mathrm{Mg}_{20} \mathrm{Cl}_{40}$ (Optimized)}

Input Keywords:

\#p opt hf/Gen geom=connectivity scfcyc=800 Input coordinates:

$\begin{array}{lccc}\mathrm{Cl} & 3.86720600 & -5.22515600 & -4.04885500 \\ \mathrm{Mg} & 3.82811400 & -2.83995100 & -3.95472100 \\ \mathrm{Mg} & 3.58767000 & -5.69641900 & -1.68110600 \\ \mathrm{Cl} & 4.23795300 & -3.17810600 & -1.45979100 \\ \mathrm{Cl} & 1.28508500 & -4.99149200 & -1.04358400 \\ \mathrm{Mg} & 1.87132900 & -2.55152100 & -0.87228700\end{array}$




\begin{tabular}{|c|c|c|c|}
\hline $\mathrm{Cl}$ & 1.51459200 & -2.16869300 & -3.36524800 \\
\hline $\mathrm{Cl}$ & 4.43104300 & -0.31164200 & -3.56782500 \\
\hline $\mathrm{Cl}$ & 4.19532500 & -2.23086500 & -6.32184900 \\
\hline $\mathrm{Mg}$ & 3.98631100 & 0.07917100 & -5.92916700 \\
\hline $\mathrm{Cl}$ & 4.03040700 & -5.92618700 & 0.83840900 \\
\hline $\mathrm{Mg}$ & 3.43465000 & -8.45467400 & 0.73212000 \\
\hline $\mathrm{Cl}$ & 3.65953300 & -8.08281600 & -1.65480700 \\
\hline $\mathrm{Mg}$ & 2.00371700 & 0.30357700 & -3.09367300 \\
\hline $\mathrm{Cl}$ & 2.15413400 & -2.90013100 & 1.59376500 \\
\hline $\mathrm{Mg}$ & 1.68250800 & -5.36602600 & 1.47530800 \\
\hline $\mathrm{Mg}$ & -0.21949300 & D -2.30414500 & 2.10140600 \\
\hline $\mathrm{Mg}$ & 2.35366800 & 3.17080000 & -5.40018100 \\
\hline $\mathrm{Cl}$ & 2.32436200 & -0.08521600 & -0.65749200 \\
\hline $\mathrm{Cl}$ & -0.54112100 & -1.91599800 & -0.33507300 \\
\hline $\mathrm{Mg}$ & -0.08857600 & 0.55024300 & -0.12123400 \\
\hline $\mathrm{Cl}$ & 1.64508300 & 0.64438100 & -5.58321900 \\
\hline $\mathrm{Cl}$ & 4.42556700 & 2.32076200 & -6.46811500 \\
\hline $\mathrm{Cl}$ & 1.06953200 & -7.78963800 & 1.28783900 \\
\hline $\mathrm{Mg}$ & 1.36481500 & -8.18971300 & 3.75121700 \\
\hline $\mathrm{Cl}$ & 3.49115600 & -10.82653700 & 0.84528500 \\
\hline $\mathrm{Cl}$ & 3.86217300 & -8.44514300 & 3.26041600 \\
\hline $\mathrm{Mg}$ & 3.53714000 & -10.96410800 & $\begin{array}{ll}0 & 3.26319100\end{array}$ \\
\hline $\mathrm{Mg}$ & -1.80442200 & ) 3.69577700 & 0.68804300 \\
\hline $\mathrm{Cl}$ & -0.79038200 & -4.74594000 & 1.97776200 \\
\hline $\mathrm{Cl}$ & 0.14003700 & -2.64480900 & 4.59084000 \\
\hline $\mathrm{Mg}$ & -2.04357800 & 0.83944800 & 2.96230300 \\
\hline $\mathrm{Mg}$ & -0.56893400 & -5.17128200 & 4.40774300 \\
\hline $\mathrm{Mg}$ & -2.20133300 & o -2.07927200 & 4.93762200 \\
\hline $\mathrm{Cl}$ & 2.52597200 & 5.50988900 & -5.77416900 \\
\hline $\mathrm{Cl}$ & 2.57367100 & 2.74494800 & -2.97016400 \\
\hline $\mathrm{Cl}$ & -0.10656800 & 3.65494100 & -4.95433200 \\
\hline $\mathrm{Cl}$ & -0.37074200 & 0.89836300 & -2.58727300 \\
\hline $\mathrm{Mg}$ & 0.41924700 & 6.18908700 & -4.74353000 \\
\hline $\mathrm{Mg}$ & 0.09950500 & 3.36418100 & -2.46885900 \\
\hline $\mathrm{Cl}$ & 0.49818900 & 2.99048400 & 0.05026600 \\
\hline $\mathrm{Cl}$ & 0.26978100 & 0.16818700 & 2.37225700 \\
\hline $\mathrm{Cl}$ & -2.45468900 & 1.17706000 & 0.46740200 \\
\hline $\mathrm{Cl}$ & -2.64648900 & -1.68915500 & 2.57620000 \\
\hline $\mathrm{Cl}$ & 1.89077000 & -5.65530500 & 3.96092600 \\
\hline $\mathrm{Cl}$ & -0.74155600 & -7.51031600 & 4.78234800 \\
\hline $\mathrm{Cl}$ & 4.97956800 & -12.24909000 & 4.37704900 \\
\hline $\mathrm{Cl}$ & 1.28497600 & -10.54032000 & 4.05827700 \\
\hline $\mathrm{Cl}$ & -2.64035500 & -4.32092900 & 5.47652700 \\
\hline $\mathrm{Cl}$ & 0.71384300 & 5.78817000 & -2.28027200 \\
\hline $\mathrm{Cl}$ & -2.24758300 & 3.92485900 & -1.83143500 \\
\hline $\mathrm{Mg}$ & -1.65109300 & 6.45441100 & -1.72506700 \\
\hline $\mathrm{Cl}$ & -1.87843400 & 6.08206300 & 0.66140400 \\
\hline $\mathrm{Cl}$ & -2.08386300 & 3.22457800 & 3.05579100 \\
\hline $\mathrm{Cl}$ & -2.41101600 & 0.23078000 & 5.32965600 \\
\hline $\mathrm{Cl}$ & -2.07846700 & 6.44492900 & -4.25250200 \\
\hline $\mathrm{Mg}$ & -1.75262400 & $\begin{array}{l}0.96402400\end{array}$ & -4.25554000 \\
\hline $\mathrm{Cl}$ & 0.49877700 & 8.53940100 & -5.05262100 \\
\hline $\mathrm{Cl}$ & -1.70588700 & 8.82644000 & -1.83770400 \\
\hline $\mathrm{Cl}$ & -3.19526300 & 10.24979200 & -5.36829500 \\
\hline
\end{tabular}

Output coordinates:

$\begin{array}{llll}\mathrm{Cl} & -2.947123 & -5.490026 & -0.892731 \\ \mathrm{Mg} & -0.810413 & -4.849900 & -0.053536 \\ \mathrm{Mg} & -4.251374 & -3.593842 & -0.113771 \\ \mathrm{Cl} & -2.144106 & -3.237331 & 1.420789 \\ \mathrm{Cl} & -3.488463 & -1.590318 & -1.378864 \\ \mathrm{Mg} & -1.428888 & -1.238310 & 0.039289\end{array}$




\begin{tabular}{|c|c|c|c|}
\hline $\mathrm{Cl}$ & -0.057930 & -2.879359 & -1.356824 \\
\hline $\mathrm{Cl}$ & 1.276203 & -4.337351 & 1.518172 \\
\hline $\mathrm{Cl}$ & 0.617833 & -6.746279 & -0.732071 \\
\hline $\mathrm{Mg}$ & 2.597178 & -5.783176 & 0.088101 \\
\hline $\mathrm{Cl}$ & -5.526939 & -1.943425 & 1.415514 \\
\hline $\mathrm{Mg}$ & -7.672539 & -2.252984 & -0.050993 \\
\hline $\mathrm{Cl}$ & -6.432364 & -4.160381 & -0.888304 \\
\hline $\mathrm{Mg}$ & 2.023215 & -2.378267 & 0.039652 \\
\hline $\mathrm{Cl}$ & -2.753304 & 0.435106 & 1.370865 \\
\hline $\mathrm{Mg}$ & -4.881895 & 0.064528 & 0.067554 \\
\hline $\mathrm{Mg}$ & -2.023017 & 2.378525 & -0.039147 \\
\hline $\mathrm{Mg}$ & 5.480965 & -3.725796 & 0.160137 \\
\hline $\mathrm{Cl}$ & 0.667396 & -0.770760 & 1.378918 \\
\hline $\mathrm{Cl}$ & -0.667606 & 0.770686 & -1.378532 \\
\hline $\mathrm{Mg}$ & 1.428864 & 1.238070 & -0.039330 \\
\hline $\mathrm{Cl}$ & 3.343641 & -4.020128 & -1.400609 \\
\hline $\mathrm{Cl}$ & 4.777178 & -5.976439 & 0.925331 \\
\hline $\mathrm{Cl}$ & -6.900293 & -0.264423 & -1.394106 \\
\hline $\mathrm{Mg}$ & -8.275547 & 1.383170 & -0.081730 \\
\hline $\mathrm{Cl}$ & -9.847487 & -2.739336 & -0.845848 \\
\hline $\mathrm{Cl}$ & -8.771687 & -0.547965 & 1.552261 \\
\hline $\mathrm{Mg}$ & -10.941779 & $9-0.916173$ & $\begin{array}{ll}3 & 0.294895\end{array}$ \\
\hline $\mathrm{Mg}$ & 4.251460 & 3.593806 & 0.113807 \\
\hline $\mathrm{Cl}$ & -4.116352 & 2.085171 & -1.415847 \\
\hline $\mathrm{Cl}$ & -3.343445 & 4.020013 & 1.401265 \\
\hline $\mathrm{Mg}$ & 0.810503 & 4.849886 & 0.053594 \\
\hline $\mathrm{Mg}$ & -5.481173 & 3.725779 & -0.160060 \\
\hline $\mathrm{Mg}$ & -2.597191 & 5.783139 & -0.087280 \\
\hline $\mathrm{Cl}$ & 7.713355 & -3.595476 & 0.943111 \\
\hline $\mathrm{Cl}$ & 4.116443 & -2.085155 & 1.416590 \\
\hline $\mathrm{Cl}$ & 6.150650 & -1.802032 & -1.384816 \\
\hline $\mathrm{Cl}$ & 2.753456 & -0.435225 & -1.370183 \\
\hline $\mathrm{Mg}$ & 8.275561 & -1.383340 & 0.080632 \\
\hline $\mathrm{Mg}$ & 4.882371 & -0.064432 & -0.065516 \\
\hline $\mathrm{Cl}$ & 3.488402 & 1.590457 & 1.379285 \\
\hline $\mathrm{Cl}$ & 0.058049 & 2.879368 & 1.357031 \\
\hline $\mathrm{Cl}$ & 2.143879 & 3.237284 & -1.420670 \\
\hline $\mathrm{Cl}$ & -1.276384 & 4.337559 & -1.517835 \\
\hline $\mathrm{Cl}$ & -6.151166 & 1.802454 & 1.385021 \\
\hline $\mathrm{Cl}$ & -7.713266 & 3.595411 & -0.943879 \\
\hline $\mathrm{Cl}$ & -12.824802 & -1.209132 & 1.424525 \\
\hline $\mathrm{Cl}$ & -10.486462 & 1.145286 & -0.880882 \\
\hline $\mathrm{Cl}$ & -4.777313 & 5.976589 & -0.924304 \\
\hline $\mathrm{Cl}$ & 6.900977 & 0.264476 & 1.394245 \\
\hline $\mathrm{Cl}$ & 5.526576 & 1.943370 & -1.415094 \\
\hline $\mathrm{Mg}$ & 7.672599 & 2.253288 & 0.050488 \\
\hline $\mathrm{Cl}$ & 6.432489 & 4.160392 & 0.888394 \\
\hline $\mathrm{Cl}$ & 2.947312 & 5.490024 & 0.892676 \\
\hline $\mathrm{Cl}$ & -0.617639 & 6.746174 & 0.732577 \\
\hline $\mathrm{Cl}$ & 8.771150 & 0.547917 & -1.553036 \\
\hline $\mathrm{Mg}$ & 10.941657 & $7 \quad 0.915980$ & -0.296358 \\
\hline $\mathrm{Cl}$ & 10.486621 & -1.145482 & 0.879472 \\
\hline $\mathrm{Cl}$ & 9.847818 & 2.739273 & 0.844663 \\
\hline $\mathrm{Cl}$ & 12.824308 & 1.209046 & -1.426572 \\
\hline
\end{tabular}

\section{$\mathrm{Mg}_{25} \mathrm{Cl}_{50}($ Optimized $)$}

Input Keywords:

\#p opt hf/Gen geom=connectivity scfcyc $=800$ Input coordinates: 


\begin{tabular}{|c|c|c|c|}
\hline $\mathrm{Cl}$ & 3.89799931 & -5.23347148 & -4.00835259 \\
\hline $\mathrm{Mg}$ & 3.79189783 & -2.85307206 & -3.94660856 \\
\hline $\mathrm{Mg}$ & 3.61859382 & -5.67825430 & -1.63042562 \\
\hline $\mathrm{Cl}$ & 4.19197183 & -3.14333102 & -1.44303574 \\
\hline $\mathrm{Cl}$ & 1.29089112 & -5.03546632 & -1.02313420 \\
\hline $\mathrm{Mg}$ & 1.80066448 & -2.58749357 & -0.85981538 \\
\hline $\mathrm{Cl}$ & 1.46722359 & -2.21397590 & -3.37980937 \\
\hline $\mathrm{Cl}$ & 4.36185227 & -0.30528072 & -3.60086443 \\
\hline $\mathrm{Cl}$ & 4.14319806 & -2.25823403 & -6.32639872 \\
\hline $\mathrm{Mg}$ & 3.91516689 & 0.05440786 & -5.96694728 \\
\hline $\mathrm{Cl}$ & 4.04604032 & -5.85369835 & 0.89242813 \\
\hline $\mathrm{Mg}$ & 3.55031732 & -8.40014354 & 0.81626756 \\
\hline $\mathrm{Cl}$ & 3.78314890 & -8.05739754 & -1.57631615 \\
\hline $\mathrm{Mg}$ & 1.92958874 & 0.26170285 & -3.14173740 \\
\hline $\mathrm{Cl}$ & 2.07249642 & -2.89512320 & 1.61889633 \\
\hline $\mathrm{Mg}$ & 1.66928775 & -5.37071723 & 1.51640522 \\
\hline $\mathrm{Mg}$ & -0.32336987 & -2.36638723 & 2.15874190 \\
\hline $\mathrm{Mg}$ & 2.26014950 & 3.14556437 & -5.46403019 \\
\hline $\mathrm{Cl}$ & 2.24778393 & -0.10589291 & -0.70220664 \\
\hline $\mathrm{Cl}$ & -0.61380561 & -1.99116779 & -0.33803867 \\
\hline $\mathrm{Mg}$ & -0.15022392 & 0.47671143 & -0.19251084 \\
\hline $\mathrm{Cl}$ & 1.56917146 & 0.61134821 & -5.63269088 \\
\hline $\mathrm{Cl}$ & 4.33337778 & 2.29971645 & -6.52626415 \\
\hline $\mathrm{Cl}$ & 1.15846284 & -7.82014078 & 1.33641663 \\
\hline $\mathrm{Mg}$ & 1.44344844 & -8.18080673 & 3.80955196 \\
\hline $\mathrm{Cl}$ & 3.71307133 & -10.76579620 & 0.95884533 \\
\hline $\mathrm{Cl}$ & 3.95832082 & -8.33788695 & 3.34486158 \\
\hline $\mathrm{Mg}$ & 3.74404267 & -10.86604440 & $\begin{array}{ll}0 & 3.37938085\end{array}$ \\
\hline $\mathrm{Mg}$ & -2.10185076 & 3.54063398 & 0.47463619 \\
\hline $\mathrm{Cl}$ & -0.81814544 & -4.81602438 & 1.97361464 \\
\hline $\mathrm{Cl}$ & -0.09649389 & -2.69729551 & 4.64991003 \\
\hline $\mathrm{Mg}$ & -2.22782252 & $2 \quad 0.69254228$ & 2.75838039 \\
\hline $\mathrm{Mg}$ & -0.64129640 & -5.23496450 & 4.42176408 \\
\hline $\mathrm{Mg}$ & -2.56094958 & $3-2.19214227$ & 5.07906462 \\
\hline $\mathrm{Cl}$ & 2.44547954 & 5.49568310 & -5.81660854 \\
\hline $\mathrm{Cl}$ & 2.47512668 & 2.72842953 & -3.03197095 \\
\hline $\mathrm{Cl}$ & -0.20411622 & 3.65059574 & -5.03483160 \\
\hline $\mathrm{Cl}$ & -0.44426381 & 0.84049246 & -2.66800784 \\
\hline $\mathrm{Mg}$ & 0.34083289 & 6.18845540 & -4.80602081 \\
\hline $\mathrm{Mg}$ & 0.02250641 & 3.31965454 & -2.54378144 \\
\hline $\mathrm{Cl}$ & 0.31294467 & 2.94477161 & -0.04679477 \\
\hline $\mathrm{Cl}$ & 0.14473100 & 0.11276150 & 2.28330843 \\
\hline $\mathrm{Cl}$ & -2.54788703 & 1.05922849 & 0.31832838 \\
\hline $\mathrm{Cl}$ & -2.77553770 & -1.77403561 & 2.64722886 \\
\hline $\mathrm{Cl}$ & 1.85846407 & -5.62611470 & 4.00063927 \\
\hline $\mathrm{Cl}$ & -0.69816285 & -7.59104900 & 4.79016474 \\
\hline $\mathrm{Cl}$ & 5.23431780 & -12.07518628 & 4.51688225 \\
\hline $\mathrm{Cl}$ & 1.46997743 & -10.53226316 & 4.14556842 \\
\hline $\mathrm{Cl}$ & -2.74629186 & -4.54221002 & 5.43142884 \\
\hline $\mathrm{Cl}$ & 0.51757560 & 5.76938379 & -2.35811281 \\
\hline $\mathrm{Cl}$ & -2.37314117 & 3.84862192 & -2.00388924 \\
\hline $\mathrm{Mg}$ & -1.96955203 & 36.32483390 & -1.90078490 \\
\hline $\mathrm{Cl}$ & -1.59210941 & 5.98894449 & 0.63819513 \\
\hline $\mathrm{Cl}$ & -1.76751641 & 3.16835385 & 2.99486207 \\
\hline $\mathrm{Cl}$ & -4.49291394 & 4.09653480 & 1.05823863 \\
\hline $\mathrm{Mg}$ & -3.91988887 & $7 \quad 6.63147936$ & 1.24545775 \\
\hline $\mathrm{Mg}$ & -4.09245046 & $5 \quad 3.80659337$ & 3.56167588 \\
\hline $\mathrm{Cl}$ & -1.86893888 & 0.34217511 & 5.24875732 \\
\hline $\mathrm{Cl}$ & -4.66116025 & 1.25885595 & 3.21610761 \\
\hline $\mathrm{Cl}$ & -4.63368677 & -1.34578596 & 6.14152009 \\
\hline $\mathrm{Mg}$ & -4.21513148 & $\begin{array}{ll}3 & 0.89948070\end{array}$ & 5.58204687 \\
\hline
\end{tabular}




\begin{tabular}{|c|c|c|c|}
\hline $\mathrm{Cl}$ & -2.15941195 & 6.57936248 & -4.38535147 \\
\hline $\mathrm{Mg}$ & -1.74568491 & 9.13374314 & -4.19547065 \\
\hline $\mathrm{Cl}$ & 0.39714673 & 8.54455004 & -5.17433520 \\
\hline $\mathrm{Cl}$ & -4.19890081 & 6.18694663 & 3.62343249 \\
\hline $\mathrm{Cl}$ & -4.44364600 & 3.21199858 & 5.94169745 \\
\hline $\mathrm{Cl}$ & -4.08640632 & 9.01056579 & 1.19056614 \\
\hline $\mathrm{Cl}$ & -4.34688571 & 6.80589976 & -1.27763199 \\
\hline $\mathrm{Mg}$ & -3.85286459 & 9.35290643 & -1.20196947 \\
\hline $\mathrm{Cl}$ & -1.46049545 & 8.77425291 & -1.72197337 \\
\hline $\mathrm{Cl}$ & -4.26145842 & 9.28920752 & -3.72943315 \\
\hline $\mathrm{Cl}$ & -4.01373842 & 11.71868960 & -1.34545115 \\
\hline $\mathrm{Cl}$ & -1.77525649 & 11.48429716 & -4.53602717 \\
\hline $\mathrm{Mg}$ & -4.04856547 & 11.81773318 & $8-3.76614369$ \\
\hline $\mathrm{Cl}$ & -5.54051959 & 13.02642938 & -4.90170802 \\
\hline \multicolumn{4}{|c|}{ Output coordinates: } \\
\hline $\mathrm{Cl}$ & 5.42020000 & 5.80911200 & -0.96405900 \\
\hline $\mathrm{Mg}$ & 3.20617300 & 5.55134800 & -0.13299800 \\
\hline $\mathrm{Mg}$ & 6.38044600 & 3.72497500 & -0.14898500 \\
\hline $\mathrm{Cl}$ & 4.23855800 & 3.75054500 & 1.36949300 \\
\hline $\mathrm{Cl}$ & 5.29825500 & 1.86542200 & -1.39939600 \\
\hline $\mathrm{Mg}$ & 3.21808000 & 1.86356100 & 0.00973200 \\
\hline $\mathrm{Cl}$ & 2.12269800 & 3.72582600 & -1.40469200 \\
\hline $\mathrm{Cl}$ & 1.05948000 & 5.44662700 & 1.45160300 \\
\hline $\mathrm{Cl}$ & 2.11304000 & 7.65588000 & -0.84523200 \\
\hline $\mathrm{Mg}$ & 0.00000100 & 7.07498000 & 0.00002300 \\
\hline $\mathrm{Cl}$ & 7.34683800 & 1.90405600 & 1.40589700 \\
\hline $\mathrm{Mg}$ & 9.52514500 & 1.84169300 & -0.03495100 \\
\hline $\mathrm{Cl}$ & 8.62913000 & 3.92137800 & -0.90560700 \\
\hline $\mathrm{Mg}$ & 0.00003800 & 3.63180300 & 0.00014400 \\
\hline $\mathrm{Cl}$ & 4.23603600 & 0.00000000 & 1.37344300 \\
\hline $\mathrm{Mg}$ & 6.39384200 & -0.00006200 & 0.07298100 \\
\hline $\mathrm{Mg}$ & 3.21804000 & -1.86368000 & 0.01011000 \\
\hline $\mathrm{Mg}$ & -3.20614800 & 5.55138400 & 0.13299900 \\
\hline $\mathrm{Cl}$ & 1.07202200 & 1.83976800 & 1.36850700 \\
\hline $\mathrm{Cl}$ & 2.12805500 & 0.00002900 & -1.36671000 \\
\hline $\mathrm{Mg}$ & 0.00000900 & 0.00005700 & 0.00005700 \\
\hline $\mathrm{Cl}$ & -1.05948100 & 5.44658100 & -1.45149800 \\
\hline $\mathrm{Cl}$ & -2.11303300 & 7.65593200 & 0.84524000 \\
\hline $\mathrm{Cl}$ & 8.44974500 & 0.00005200 & -1.37402700 \\
\hline $\mathrm{Mg}$ & 9.52506900 & -1.84176600 & -0.03537700 \\
\hline $\mathrm{Cl}$ & 11.76118700 & 1.96621500 & -0.80075200 \\
\hline $\mathrm{Cl}$ & 10.31115600 & 0.00021500 & 1.59645600 \\
\hline $\mathrm{Mg}$ & 12.52718900 & $\begin{array}{ll}0 & 0.00013100\end{array}$ & 0.37057000 \\
\hline $\mathrm{Mg}$ & -3.21806300 & -1.86357500 & -0.00994400 \\
\hline $\mathrm{Cl}$ & 5.29823800 & -1.86545000 & -1.39930300 \\
\hline $\mathrm{Cl}$ & 4.23851800 & -3.75059200 & 1.36963800 \\
\hline $\mathrm{Mg}$ & -0.00008100 & -3.63188700 & 0.00005300 \\
\hline $\mathrm{Mg}$ & 6.38068300 & -3.72506100 & -0.14930500 \\
\hline $\mathrm{Mg}$ & 3.20628300 & -5.55132200 & -0.13262900 \\
\hline $\mathrm{Cl}$ & -5.42016600 & 5.80917500 & 0.96408600 \\
\hline $\mathrm{Cl}$ & -2.12271300 & 3.72583400 & 1.40477400 \\
\hline $\mathrm{Cl}$ & -4.23858200 & 3.75052100 & -1.36942500 \\
\hline $\mathrm{Cl}$ & -1.07193500 & 1.83978900 & -1.36832300 \\
\hline $\mathrm{Mg}$ & -6.38044400 & 3.72506100 & 0.14913600 \\
\hline $\mathrm{Mg}$ & -3.21808500 & 1.86354200 & -0.00974600 \\
\hline $\mathrm{Cl}$ & -2.12801700 & -0.00002500 & 1.36683600 \\
\hline $\mathrm{Cl}$ & 1.07188000 & -1.83972700 & 1.36844300 \\
\hline $\mathrm{Cl}$ & -1.07191300 & -1.83973600 & -1.36837500 \\
\hline $\mathrm{Cl}$ & 2.12276800 & -3.72591500 & -1.40452400 \\
\hline $\mathrm{Cl}$ & 7.34707400 & -1.90458100 & 1.40573600 \\
\hline $\mathrm{Cl}$ & 8.62909800 & -3.92112700 & -0.90677100 \\
\hline
\end{tabular}




\begin{tabular}{|c|c|c|c|}
\hline $\mathrm{Cl}$ & 14.41871500 & 0.00018200 & 1.52562000 \\
\hline $\mathrm{Cl}$ & 11.76133500 & -1.96638700 & -0.80020600 \\
\hline $\mathrm{Cl}$ & 5.42031500 & -5.80923700 & -0.96378800 \\
\hline $\mathrm{Cl}$ & -5.29823300 & 1.86545100 & 1.39949300 \\
\hline $\mathrm{Cl}$ & -4.23608100 & 0.00003900 & -1.37321800 \\
\hline $\mathrm{Mg}$ & -6.39399500 & -0.00004400 & -0.07245500 \\
\hline $\mathrm{Cl}$ & -5.29832100 & -1.86553000 & 1.39946000 \\
\hline $\mathrm{Cl}$ & -2.12282100 & -3.72586200 & 1.40470100 \\
\hline $\mathrm{Cl}$ & -4.23838700 & -3.75056800 & -1.36957600 \\
\hline $\mathrm{Mg}$ & -6.38065300 & -3.72499000 & 0.14904900 \\
\hline $\mathrm{Mg}$ & -3.20627800 & -5.55127500 & 0.13279500 \\
\hline $\mathrm{Cl}$ & 1.05931500 & -5.44650100 & 1.45180100 \\
\hline $\mathrm{Cl}$ & -1.05934400 & -5.44661700 & -1.45157800 \\
\hline $\mathrm{Cl}$ & 2.11316800 & -7.65589600 & -0.84472700 \\
\hline $\mathrm{Mg}$ & -0.00000600 & -7.07489600 & 0.00019700 \\
\hline $\mathrm{Cl}$ & -7.34692000 & 1.90415100 & -1.40578100 \\
\hline $\mathrm{Mg}$ & -9.52497900 & 1.84177500 & 0.03473300 \\
\hline $\mathrm{Cl}$ & -8.62904200 & 3.92127900 & 0.90600600 \\
\hline $\mathrm{Cl}$ & -5.42038500 & -5.80917100 & 0.96376000 \\
\hline $\mathrm{Cl}$ & -2.11318900 & -7.65580100 & 0.84515100 \\
\hline $\mathrm{Cl}$ & -8.62927300 & -3.92127600 & 0.90597800 \\
\hline $\mathrm{Cl}$ & -7.34672100 & -1.90432200 & -1.40581100 \\
\hline $\mathrm{Mg}$ & -9.52523500 & -1.84178100 & 0.03500500 \\
\hline $\mathrm{Cl}$ & -8.44996300 & 0.00000300 & 1.37402000 \\
\hline $\mathrm{Cl}$ & -10.31101400 & -0.00018500 & -1.59674500 \\
\hline $\mathrm{Cl}$ & -11.76139600 & -1.96610500 & 0.80030300 \\
\hline $\mathrm{Cl}$ & -11.76122300 & 1.96653100 & 0.79978600 \\
\hline $\mathrm{Mg}$ & -12.52714600 & $\begin{array}{ll}0 & 0.00010800\end{array}$ & -0.37103700 \\
\hline $\mathrm{Cl}$ & -14.41858900 & -0.00003500 & -1.52622100 \\
\hline
\end{tabular}

\section{$\mathrm{Mg}_{25} \mathrm{Cl}_{50}$ (Frozen)}

Input Keywords:

\# hf/Gen geom=connectivity scfcyc=800 maxdisk=40gb Input coordinates:

$\begin{array}{lccc}\mathrm{Cl} & -17.98247800 & -7.95491400 & -1.36300000 \\ \mathrm{Cl} & -17.98247800 & -4.31391400 & -1.36300000 \\ \mathrm{Cl} & -17.98247800 & -0.67291400 & -1.36300000 \\ \mathrm{Cl} & -17.98247800 & 2.96808600 & -1.36300000 \\ \mathrm{Cl} & -17.98247800 & 6.60908600 & -1.36300000 \\ \mathrm{Cl} & -15.88047800 & -11.59591400 & 1.36300000 \\ \mathrm{Mg} & -16.93147800 & -9.77491400 & 0.00000000 \\ \mathrm{Cl} & -15.88047800 & -7.95491400 & 1.36300000 \\ \mathrm{Cl} & -14.82947800 & -9.77491400 & -1.36300000 \\ \mathrm{Mg} & -16.93147800 & -6.13391400 & 0.00000000 \\ \mathrm{Cl} & -15.88047800 & -4.31391400 & 1.36300000 \\ \mathrm{Cl} & -14.82947800 & -6.13391400 & -1.36300000 \\ \mathrm{Mg} & -16.93147800 & -2.49291400 & 0.00000000 \\ \mathrm{Cl} & -15.88047800 & -0.67291400 & 1.36300000 \\ \mathrm{Cl} & -14.82947800 & -2.49291400 & -1.36300000 \\ \mathrm{Mg} & -16.93147800 & 1.14808600 & 0.00000000 \\ \mathrm{Cl} & -15.88047800 & 2.96808600 & 1.36300000 \\ \mathrm{Cl} & -14.82947800 & 1.14808600 & -1.36300000 \\ \mathrm{Mg} & -16.93147800 & 4.78908600 & 0.00000000 \\ \mathrm{Cl} & -14.82947800 & 4.78908600 & -1.36300000 \\ \mathrm{Cl} & -12.72747800 & -13.41691400 & 1.36300000 \\ \mathrm{Mg} & -13.77847800 & -11.59591400 & 0.00000000 \\ \mathrm{Cl} & -12.72747800 & -9.77491400 & 1.36300000 \\ \mathrm{Cl} & -11.67547800 & -11.59591400 & -1.36300000 \\ \mathrm{Mg} & -13.77847800 & -7.95491400 & 0.00000000\end{array}$




\begin{tabular}{|c|c|}
\hline $\mathrm{Cl}$ & $-12.72747800-6.13391400$ \\
\hline $\mathrm{Cl}$ & $\begin{array}{llll}-11.67547800 & -7.95491400 & -1.36300000\end{array}$ \\
\hline $\mathrm{Mg}$ & $-13.77847800 \quad-4.31391400 \quad 0.00000000$ \\
\hline $\mathrm{Cl}$ & $\begin{array}{lll}-12.72747800 & -2.49291400 & 1.36300000\end{array}$ \\
\hline $\mathrm{Cl}$ & $-11.67547800-4.31391400$ \\
\hline $\mathrm{Mg}$ & $-13.77847800 \quad-0.67291400 \quad 0.00000000$ \\
\hline $\mathrm{Cl}$ & $\begin{array}{lll}-12.72747800 & 1.14808600 & 1.36300000\end{array}$ \\
\hline $\mathrm{Cl}$ & $\begin{array}{llll}-11.67547800 & -0.67291400 & -1.36300000\end{array}$ \\
\hline $\mathrm{Mg}$ & $\begin{array}{lll}-13.77847800 & 2.96808600 & 0.00000000\end{array}$ \\
\hline $\mathrm{Cl}$ & $\begin{array}{lll}-11.67547800 & 2.96808600 & -1.36300000\end{array}$ \\
\hline $\mathrm{Cl}$ & $-9.57347800 \quad-15.23691400 \quad 1.36300000$ \\
\hline $\mathrm{Mg}$ & $-10.62447800-13.41691400 \quad 0.00000000$ \\
\hline $\mathrm{Cl}$ & $\begin{array}{lll}-9.57347800 & -11.59591400 & 1.36300000\end{array}$ \\
\hline $\mathrm{Cl}$ & $\begin{array}{lll}-8.52247800 & -13.41691400 & -1.36300000\end{array}$ \\
\hline $\mathrm{Mg}$ & $\begin{array}{lll}-10.62447800 & -9.77491400 & 0.00000000\end{array}$ \\
\hline $\mathrm{Cl}$ & $\begin{array}{lll}-9.57347800 & -7.95491400 & 1.36300000\end{array}$ \\
\hline $\mathrm{Cl}$ & $\begin{array}{lll}-8.52247800 & -9.77491400 & -1.36300000\end{array}$ \\
\hline $\mathrm{Mg}$ & $\begin{array}{lll}-10.62447800 & -6.13391400 & 0.00000000\end{array}$ \\
\hline $\mathrm{Cl}$ & $\begin{array}{lll}-9.57347800 & -4.31391400 & 1.36300000\end{array}$ \\
\hline $\mathrm{Cl}$ & $\begin{array}{lll}-8.52247800 & -6.13391400 & -1.36300000\end{array}$ \\
\hline $\mathrm{Mg}$ & $\begin{array}{lll}-10.62447800 & -2.49291400 & 0.00000000\end{array}$ \\
\hline $\mathrm{Cl}$ & $\begin{array}{lll}-9.57347800 & -0.67291400 & 1.36300000\end{array}$ \\
\hline $\mathrm{Cl}$ & $\begin{array}{lll}-8.52247800 & -2.49291400 & -1.36300000\end{array}$ \\
\hline $\mathrm{Mg}$ & $\begin{array}{lll}-10.62447800 & 1.14808600 & 0.00000000\end{array}$ \\
\hline $\mathrm{Cl}$ & $\begin{array}{lll}-8.52247800 & 1.14808600 & -1.36300000\end{array}$ \\
\hline $\mathrm{Cl}$ & $-6.42047800-17.05791400 \quad 1.36300000$ \\
\hline $\mathrm{Mg}$ & $-7.47147800-15.23691400 \quad 0.00000000$ \\
\hline $\mathrm{Cl}$ & $-6.42047800-13.41691400 \quad 1.36300000$ \\
\hline $\mathrm{Cl}$ & $\begin{array}{lll}-5.36947800 & -15.23691400 & -1.36300000\end{array}$ \\
\hline $\mathrm{Mg}$ & $-7.47147800-11.59591400 \quad 0.00000000$ \\
\hline $\mathrm{Cl}$ & $-6.42047800 \quad-9.77491400 \quad 1.36300000$ \\
\hline $\mathrm{Cl}$ & $\begin{array}{lll}-5.36947800 & -11.59591400 & -1.36300000\end{array}$ \\
\hline $\mathrm{Mg}$ & $-7.47147800 \quad-7.95491400 \quad 0.00000000$ \\
\hline $\mathrm{Cl}$ & $-6.42047800 \quad-6.13391400 \quad 1.36300000$ \\
\hline $\mathrm{Cl}$ & $\begin{array}{lll}-5.36947800 & -7.95491400 & -1.36300000\end{array}$ \\
\hline $\mathrm{Mg}$ & $-7.47147800 \quad-4.31391400 \quad 0.00000000$ \\
\hline $\mathrm{Cl}$ & $-6.42047800 \quad-2.49291400 \quad 1.36300000$ \\
\hline $\mathrm{Cl}$ & $\begin{array}{lll}-5.36947800 & -4.31391400 & -1.36300000\end{array}$ \\
\hline $\mathrm{Mg}$ & $-7.47147800 \quad-0.67291400 \quad 0.00000000$ \\
\hline $\mathrm{Cl}$ & $\begin{array}{lll}-5.36947800 & -0.67291400 & -1.36300000\end{array}$ \\
\hline $\mathrm{Cl}$ & $-3.26747800-18.87791400 \quad 1.36300000$ \\
\hline $\mathrm{Mg}$ & $-4.31847800 \quad-17.05791400 \quad 0.00000000$ \\
\hline $\mathrm{Cl}$ & $-3.26747800-15.23691400 \quad 1.36300000$ \\
\hline $\mathrm{Mg}$ & $-4.31847800-13.41691400 \quad 0.00000000$ \\
\hline $\mathrm{Cl}$ & $-3.26747800-11.59591400 \quad 1.36300000$ \\
\hline $\mathrm{Mg}$ & $-4.31847800 \quad-9.77491400 \quad 0.00000000$ \\
\hline $\mathrm{Cl}$ & $\begin{array}{lll}-3.26747800 & -7.95491400 & 1.36300000\end{array}$ \\
\hline $\mathrm{Mg}$ & $-4.31847800 \quad-6.13391400 \quad 0.00000000$ \\
\hline $\mathrm{Cl}$ & $-3.26747800 \quad-4.31391400 \quad 1.36300000$ \\
\hline $\mathrm{Mg}$ & $-4.31847800 \quad-2.49291400 \quad 0.00000000$ \\
\hline
\end{tabular}

\section{$\mathrm{Mg}_{36} \mathrm{Cl}_{72}$ (Optimized)}

Input Keywords:

\#p opt hf/Gen geom=connectivity scfcyc $=800$ Input coordinates:

$\begin{array}{lrrr}\mathrm{Cl} & 0.90629200 & 2.09479300 & 9.47399000 \\ \mathrm{Cl} & 1.41084000 & -1.09899100 & 7.25347700 \\ \mathrm{Cl} & 1.27393200 & -4.26001000 & 5.57169100 \\ \mathrm{Cl} & 1.26586800 & -7.38001200 & 3.75324900\end{array}$




\begin{tabular}{|c|c|c|c|}
\hline $\mathrm{Cl}$ & 1.39493900 & -10.44708500 & 1.90388700 \\
\hline $\mathrm{Cl}$ & 1.71809500 & -13.35503700 & 0.00000000 \\
\hline $\mathrm{Cl}$ & 1.06564400 & 5.39142900 & 7.63640800 \\
\hline $\mathrm{Cl}$ & 1.43471700 & 2.08609000 & 5.53308000 \\
\hline $\mathrm{Cl}$ & 1.34704800 & -1.08980600 & 3.66428700 \\
\hline $\mathrm{Cl}$ & 1.29809200 & -4.24806800 & 1.85317100 \\
\hline $\mathrm{Cl}$ & 1.31718200 & -7.36311200 & 0.00000000 \\
\hline $\mathrm{Cl}$ & 1.39493900 & -10.44708500 & -1.90388700 \\
\hline $\mathrm{Cl}$ & 1.07767700 & 8.60870700 & 5.76009100 \\
\hline $\mathrm{Cl}$ & 1.48244400 & 5.26889700 & 3.69348200 \\
\hline $\mathrm{Cl}$ & 1.39911100 & 2.09873000 & 1.85403100 \\
\hline $\mathrm{Cl}$ & 1.34317800 & -1.09048500 & 0.00000000 \\
\hline $\mathrm{Cl}$ & 1.29809200 & -4.24806800 & -1.85317100 \\
\hline $\mathrm{Cl}$ & 1.26586800 & -7.38001200 & -3.75324900 \\
\hline $\mathrm{Cl}$ & 0.88457100 & 11.81240900 & 3.89276800 \\
\hline $\mathrm{Cl}$ & 1.43731000 & 8.46717900 & 1.83057300 \\
\hline $\mathrm{Cl}$ & 1.42582900 & 5.29359200 & 0.00000000 \\
\hline $\mathrm{Cl}$ & 1.39911100 & 2.09873000 & -1.85403100 \\
\hline $\mathrm{Cl}$ & 1.34704800 & -1.08980600 & -3.66428700 \\
\hline $\mathrm{Cl}$ & 1.27393200 & -4.26001000 & -5.57169100 \\
\hline $\mathrm{Cl}$ & 0.57927400 & 14.95505600 & 1.95013600 \\
\hline $\mathrm{Cl}$ & 1.31612200 & 11.63353600 & 0.00000000 \\
\hline $\mathrm{Cl}$ & 1.43731000 & 8.46717900 & -1.83057300 \\
\hline $\mathrm{Cl}$ & 1.48244400 & $5.26889700-$ & -3.69348200 \\
\hline $\mathrm{Cl}$ & 1.43471700 & $2.08609000-$ & -5.53308000 \\
\hline $\mathrm{Cl}$ & 1.41084000 & -1.09899100 & -7.25347700 \\
\hline $\mathrm{Cl}$ & -1.98412600 & 17.46704100 & 0.00000000 \\
\hline $\mathrm{Cl}$ & 0.57927400 & 14.95505600 & -1.95013600 \\
\hline $\mathrm{Cl}$ & 0.88457100 & 11.81240900 & -3.89276800 \\
\hline $\mathrm{Cl}$ & 1.07767700 & 8.60870700 & -5.76009100 \\
\hline $\mathrm{Cl}$ & 1.06564400 & $5.39142900-$ & -7.63640800 \\
\hline $\mathrm{Cl}$ & 0.90629200 & $2.09479300-$ & -9.47399000 \\
\hline $\mathrm{Mg}$ & 0.00000000 & 0.00000000 & 8.90529000 \\
\hline $\mathrm{Mg}$ & -0.19143900 & -3.18740300 & 7.36770300 \\
\hline $\mathrm{Mg}$ & -0.25875900 & -6.37048800 & 5.55884700 \\
\hline $\mathrm{Mg}$ & -0.19041100 & -9.53470700 & 3.70467700 \\
\hline $\mathrm{Mg}$ & 0.06117600 & -12.66857200 & 1.83134400 \\
\hline $\mathrm{Mg}$ & 0.66987500 & -15.66306100 & 0.00000000 \\
\hline $\mathrm{Mg}$ & 0.19143900 & 3.18740300 & 7.36770300 \\
\hline $\mathrm{Mg}$ & 0.00000000 & 0.00000000 & 5.44595300 \\
\hline $\mathrm{Mg}$ & -0.03565800 & -3.20356700 & 3.68976300 \\
\hline $\mathrm{Mg}$ & -0.06008400 & -6.37322200 & 1.86169800 \\
\hline $\mathrm{Mg}$ & 0.05170600 & -9.52676800 & 0.00000000 \\
\hline $\mathrm{Mg}$ & 0.06117600 & -12.66857200 & -1.83134400 \\
\hline $\mathrm{Mg}$ & 0.25875900 & 6.37048800 & 5.55884700 \\
\hline $\mathrm{Mg}$ & 0.03565800 & 3.20356700 & 3.68976300 \\
\hline $\mathrm{Mg}$ & 0.00000000 & 0.00000000 & 1.82197000 \\
\hline $\mathrm{Mg}$ & -0.07291400 & -3.19330100 & 0.00000000 \\
\hline $\mathrm{Mg}$ & -0.06008400 & -6.37322200 & -1.86169800 \\
\hline $\mathrm{Mg}$ & -0.19041100 & -9.53470700 & -3.70467700 \\
\hline $\mathrm{Mg}$ & 0.19041100 & 9.53470700 & 3.70467700 \\
\hline $\mathrm{Mg}$ & 0.06008400 & 6.37322200 & 1.86169800 \\
\hline $\mathrm{Mg}$ & 0.07291400 & 3.19330100 & 0.00000000 \\
\hline $\mathrm{Mg}$ & 0.00000000 & 0.00000000 & -1.82197000 \\
\hline $\mathrm{Mg}$ & -0.03565800 & -3.20356700 & -3.68976300 \\
\hline $\mathrm{Mg}$ & -0.25875900 & -6.37048800 & -5.55884700 \\
\hline $\mathrm{Mg}$ & -0.06117600 & 12.66857200 & 1.83134400 \\
\hline $\mathrm{Mg}$ & -0.05170600 & 9.52676800 & 0.00000000 \\
\hline $\mathrm{Mg}$ & 0.06008400 & 6.37322200 & -1.86169800 \\
\hline $\mathrm{Mg}$ & 0.03565800 & 3.20356700 & -3.68976300 \\
\hline $\mathrm{Mg}$ & 0.00000000 & 0.00000000 & -5.44595300 \\
\hline
\end{tabular}




\begin{tabular}{|c|c|c|c|}
\hline $\mathrm{Mg}$ & -0.19143900 & -3.18740300 & -7.36770300 \\
\hline $\mathrm{Mg}$ & -0.66987500 & 15.66306100 & 0.00000000 \\
\hline $\mathrm{Mg}$ & -0.06117600 & 12.66857200 & -1.83134400 \\
\hline $\mathrm{Mg}$ & 0.19041100 & 9.53470700 & -3.70467700 \\
\hline $\mathrm{Mg}$ & 0.25875900 & 6.37048800 & -5.55884700 \\
\hline $\mathrm{Mg}$ & 0.19143900 & 3.18740300 & -7.36770300 \\
\hline $\mathrm{Mg}$ & 0.00000000 & 0.00000000 & -8.90529000 \\
\hline $\mathrm{Cl}$ & -0.90629200 & -2.09479300 & 9.47399000 \\
\hline $\mathrm{Cl}$ & -1.06564400 & -5.39142900 & 7.63640800 \\
\hline $\mathrm{Cl}$ & -1.07767700 & -8.60870700 & 5.76009100 \\
\hline $\mathrm{Cl}$ & -0.88457100 & -11.81240900 & 3.89276800 \\
\hline $\mathrm{Cl}$ & -0.57927400 & -14.95505600 & 1.95013600 \\
\hline $\mathrm{Cl}$ & 1.98412600 & -17.46704100 & 0.00000000 \\
\hline $\mathrm{Cl}$ & -1.41084000 & 1.09899100 & 7.25347700 \\
\hline $\mathrm{Cl}$ & -1.43471700 & -2.08609000 & 5.53308000 \\
\hline $\mathrm{Cl}$ & -1.48244400 & -5.26889700 & 3.69348200 \\
\hline $\mathrm{Cl}$ & -1.43731000 & -8.46717900 & 1.83057300 \\
\hline $\mathrm{Cl}$ & -1.31612200 & -11.63353600 & 0.00000000 \\
\hline $\mathrm{Cl}$ & -0.57927400 & -14.95505600 & -1.95013600 \\
\hline $\mathrm{Cl}$ & -1.27393200 & 4.26001000 & 5.57169100 \\
\hline $\mathrm{Cl}$ & -1.34704800 & 1.08980600 & 3.66428700 \\
\hline $\mathrm{Cl}$ & -1.39911100 & -2.09873000 & 1.85403100 \\
\hline $\mathrm{Cl}$ & -1.42582900 & -5.29359200 & 0.00000000 \\
\hline $\mathrm{Cl}$ & -1.43731000 & -8.46717900 & -1.83057300 \\
\hline $\mathrm{Cl}$ & -0.88457100 & -11.81240900 & -3.89276800 \\
\hline $\mathrm{Cl}$ & -1.26586800 & 7.38001200 & 3.75324900 \\
\hline $\mathrm{Cl}$ & -1.29809200 & 4.24806800 & 1.85317100 \\
\hline $\mathrm{Cl}$ & -1.34317800 & 1.09048500 & 0.00000000 \\
\hline $\mathrm{Cl}$ & -1.39911100 & -2.09873000 & -1.85403100 \\
\hline $\mathrm{Cl}$ & -1.48244400 & -5.26889700 & -3.69348200 \\
\hline $\mathrm{Cl}$ & -1.07767700 & -8.60870700 & -5.76009100 \\
\hline $\mathrm{Cl}$ & -1.39493900 & 10.44708500 & 1.90388700 \\
\hline $\mathrm{Cl}$ & -1.31718200 & 7.36311200 & 0.00000000 \\
\hline $\mathrm{Cl}$ & -1.29809200 & 4.24806800 & -1.85317100 \\
\hline $\mathrm{Cl}$ & -1.34704800 & 1.08980600 & -3.66428700 \\
\hline $\mathrm{Cl}$ & -1.43471700 & -2.08609000 & -5.53308000 \\
\hline $\mathrm{Cl}$ & -1.06564400 & -5.39142900 & -7.63640800 \\
\hline $\mathrm{Cl}$ & -1.71809500 & 13.35503700 & 0.00000000 \\
\hline $\mathrm{Cl}$ & -1.39493900 & 10.44708500 & -1.90388700 \\
\hline $\mathrm{Cl}$ & -1.26586800 & 7.38001200 & -3.75324900 \\
\hline $\mathrm{Cl}$ & -1.27393200 & 4.26001000 & -5.57169100 \\
\hline $\mathrm{Cl}$ & -1.41084000 & 1.09899100 & -7.25347700 \\
\hline $\mathrm{Cl}$ & -0.90629200 & -2.09479300 & -9.47399000 \\
\hline \multicolumn{4}{|c|}{ Output coordinates: } \\
\hline $\mathrm{Cl}$ & 5.42020000 & 5.80911200 & -0.96405900 \\
\hline $\mathrm{Mg}$ & 3.20617300 & 5.55134800 & -0.13299800 \\
\hline $\mathrm{Mg}$ & 6.38044600 & 3.72497500 & -0.14898500 \\
\hline $\mathrm{Cl}$ & 4.23855800 & 3.75054500 & 1.36949300 \\
\hline $\mathrm{Cl}$ & 5.29825500 & 1.86542200 & -1.39939600 \\
\hline $\mathrm{Mg}$ & 3.21808000 & 1.86356100 & 0.00973200 \\
\hline $\mathrm{Cl}$ & 2.12269800 & 3.72582600 & -1.40469200 \\
\hline $\mathrm{Cl}$ & 1.05948000 & 5.44662700 & 1.45160300 \\
\hline $\mathrm{Cl}$ & 2.11304000 & 7.65588000 & -0.84523200 \\
\hline $\mathrm{Mg}$ & 0.00000100 & 7.07498000 & 0.00002300 \\
\hline $\mathrm{Cl}$ & 7.34683800 & 1.90405600 & 1.40589700 \\
\hline $\mathrm{Mg}$ & 9.52514500 & 1.84169300 & -0.03495100 \\
\hline $\mathrm{Cl}$ & 8.62913000 & 3.92137800 & -0.90560700 \\
\hline $\mathrm{Mg}$ & 0.00003800 & 3.63180300 & 0.00014400 \\
\hline $\mathrm{Cl}$ & 4.23603600 & 0.00000000 & 1.37344300 \\
\hline $\mathrm{Mg}$ & 6.39384200 & -0.00006200 & 0.07298100 \\
\hline $\mathrm{Mg}$ & 3.21804000 & -1.86368000 & 0.01011000 \\
\hline
\end{tabular}




\begin{tabular}{|c|c|c|c|}
\hline $\mathrm{Mg}$ & -3.20614800 & 5.55138400 & 0.13299900 \\
\hline $\mathrm{Cl}$ & 1.07202200 & 1.83976800 & 1.36850700 \\
\hline $\mathrm{Cl}$ & 2.12805500 & 0.00002900 & -1.36671000 \\
\hline $\mathrm{Mg}$ & 0.00000900 & 0.00005700 & 0.00005700 \\
\hline $\mathrm{Cl}$ & -1.05948100 & 5.44658100 & -1.45149800 \\
\hline $\mathrm{Cl}$ & -2.11303300 & 7.65593200 & 0.84524000 \\
\hline $\mathrm{Cl}$ & 8.44974500 & 0.00005200 & -1.37402700 \\
\hline $\mathrm{Mg}$ & 9.52506900 & -1.84176600 & -0.03537700 \\
\hline $\mathrm{Cl}$ & 11.76118700 & 1.96621500 & -0.80075200 \\
\hline $\mathrm{Cl}$ & 10.31115600 & 0.00021500 & 1.59645600 \\
\hline $\mathrm{Mg}$ & 12.52718900 & 0.00013100 & 0.37057000 \\
\hline $\mathrm{Mg}$ & -3.21806300 & -1.86357500 & -0.00994400 \\
\hline $\mathrm{Cl}$ & 5.29823800 & -1.86545000 & -1.39930300 \\
\hline $\mathrm{Cl}$ & 4.23851800 & -3.75059200 & 1.36963800 \\
\hline $\mathrm{Mg}$ & -0.00008100 & -3.63188700 & 0.00005300 \\
\hline $\mathrm{Mg}$ & 6.38068300 & -3.72506100 & -0.14930500 \\
\hline $\mathrm{Mg}$ & 3.20628300 & -5.55132200 & -0.13262900 \\
\hline $\mathrm{Cl}$ & -5.42016600 & 5.80917500 & 0.96408600 \\
\hline $\mathrm{Cl}$ & -2.12271300 & 3.72583400 & 1.40477400 \\
\hline $\mathrm{Cl}$ & -4.23858200 & 3.75052100 & -1.36942500 \\
\hline $\mathrm{Cl}$ & -1.07193500 & 1.83978900 & -1.36832300 \\
\hline $\mathrm{Mg}$ & -6.38044400 & 3.72506100 & 0.14913600 \\
\hline $\mathrm{Mg}$ & -3.21808500 & 1.86354200 & -0.00974600 \\
\hline $\mathrm{Cl}$ & -2.12801700 & -0.00002500 & 1.36683600 \\
\hline $\mathrm{Cl}$ & 1.07188000 & -1.83972700 & 1.36844300 \\
\hline $\mathrm{Cl}$ & -1.07191300 & -1.83973600 & -1.36837500 \\
\hline $\mathrm{Cl}$ & 2.12276800 & -3.72591500 & -1.40452400 \\
\hline $\mathrm{Cl}$ & 7.34707400 & -1.90458100 & 1.40573600 \\
\hline $\mathrm{Cl}$ & 8.62909800 & -3.92112700 & -0.90677100 \\
\hline $\mathrm{Cl}$ & 14.41871500 & 0.00018200 & 1.52562000 \\
\hline $\mathrm{Cl}$ & 11.76133500 & -1.96638700 & -0.80020600 \\
\hline $\mathrm{Cl}$ & 5.42031500 & -5.80923700 & -0.96378800 \\
\hline $\mathrm{Cl}$ & -5.29823300 & 1.86545100 & 1.39949300 \\
\hline $\mathrm{Cl}$ & -4.23608100 & 0.00003900 & -1.37321800 \\
\hline $\mathrm{Mg}$ & -6.39399500 & -0.00004400 & -0.07245500 \\
\hline $\mathrm{Cl}$ & -5.29832100 & -1.86553000 & 1.39946000 \\
\hline $\mathrm{Cl}$ & -2.12282100 & -3.72586200 & 1.40470100 \\
\hline $\mathrm{Cl}$ & -4.23838700 & -3.75056800 & -1.36957600 \\
\hline $\mathrm{Mg}$ & -6.38065300 & -3.72499000 & 0.14904900 \\
\hline $\mathrm{Mg}$ & -3.20627800 & -5.55127500 & 0.13279500 \\
\hline $\mathrm{Cl}$ & 1.05931500 & -5.44650100 & 1.45180100 \\
\hline $\mathrm{Cl}$ & -1.05934400 & -5.44661700 & -1.45157800 \\
\hline $\mathrm{Cl}$ & 2.11316800 & -7.65589600 & -0.84472700 \\
\hline $\mathrm{Mg}$ & -0.00000600 & -7.07489600 & 0.00019700 \\
\hline $\mathrm{Cl}$ & -7.34692000 & 1.90415100 & -1.40578100 \\
\hline $\mathrm{Mg}$ & -9.52497900 & 1.84177500 & 0.03473300 \\
\hline $\mathrm{Cl}$ & -8.62904200 & 3.92127900 & 0.90600600 \\
\hline $\mathrm{Cl}$ & -5.42038500 & -5.80917100 & 0.96376000 \\
\hline $\mathrm{Cl}$ & -2.11318900 & -7.65580100 & 0.84515100 \\
\hline $\mathrm{Cl}$ & -8.62927300 & -3.92127600 & 0.90597800 \\
\hline $\mathrm{Cl}$ & -7.34672100 & -1.90432200 & -1.40581100 \\
\hline $\mathrm{Mg}$ & -9.52523500 & -1.84178100 & 0.03500500 \\
\hline $\mathrm{Cl}$ & -8.44996300 & 0.00000300 & 1.37402000 \\
\hline $\mathrm{Cl}$ & -10.31101400 & -0.00018500 & -1.59674500 \\
\hline $\mathrm{Cl}$ & -11.76139600 & -1.96610500 & 0.80030300 \\
\hline $\mathrm{Cl}$ & -11.76122300 & 1.96653100 & 0.79978600 \\
\hline $\mathrm{Mg}$ & -12.52714600 & 0.00010800 & -0.37103700 \\
\hline $\mathrm{Cl}$ & -14.41858900 & -0.00003500 & -1.52622100 \\
\hline
\end{tabular}

\section{$\mathrm{Mg}_{36} \mathrm{Cl}_{72}$ (Frozen)}


Input Keywords:

$\#$ p hf/Gen geom=connectivity scfcyc $=800$

Input coordinates:

$\mathrm{Cl} \quad-17.98247800 \quad-7.95491400 \quad-1.36300000$

$\mathrm{Cl} \quad-17.98247800 \quad-4.31391400 \quad-1.36300000$

$\mathrm{Cl} \quad-17.98247800 \quad-0.67291400 \quad-1.36300000$

$\begin{array}{lllll}\mathrm{Cl} & -17.98247800 & 2.96808600 & -1.36300000\end{array}$

$\begin{array}{lllll}\mathrm{Cl} & -17.98247800 & 6.60908600 & -1.36300000\end{array}$

$\begin{array}{lllll}\mathrm{Cl} & & -17.98247800 & 10.25008600 & -1.36300000\end{array}$

$\begin{array}{llll}\mathrm{Cl} & -15.88047800 & -11.59591400 & 1.36300000\end{array}$

$\begin{array}{llll}\mathrm{Mg} & -16.93147800 & -9.77491400 & 0.00000000\end{array}$

$\mathrm{Cl} \quad-15.88047800 \quad-7.95491400 \quad 1.36300000$

$\mathrm{Cl} \quad \quad \quad-14.82947800 \quad-9.77491400 \quad-1.36300000$

$\begin{array}{lllll}\mathrm{Mg} & -16.93147800 & -6.13391400 & 0.00000000\end{array}$

$\mathrm{Cl} \quad-15.88047800 \quad-4.31391400 \quad 1.36300000$

$\mathrm{Cl} \quad-14.82947800 \quad-6.13391400 \quad-1.36300000$

$\begin{array}{lllll}\mathrm{Mg} & & -16.93147800 & -2.49291400 & 0.00000000\end{array}$

$\begin{array}{llll}\mathrm{Cl} & -15.88047800 & -0.67291400 & 1.36300000\end{array}$

$\mathrm{Cl} \quad-14.82947800 \quad-2.49291400 \quad-1.36300000$

$\begin{array}{lllll}\mathrm{Mg} & & -16.93147800 & 1.14808600 & 0.00000000\end{array}$

$\begin{array}{llll}\mathrm{Cl} & -15.88047800 & 2.96808600 & 1.36300000\end{array}$

$\begin{array}{lllll}\mathrm{Cl} & & -14.82947800 & 1.14808600 & -1.36300000\end{array}$

$\begin{array}{lllll}\mathrm{Mg} & -16.93147800 & 4.78908600 & 0.00000000\end{array}$

$\begin{array}{lllll}\mathrm{Cl} & -15.88047800 & 6.60908600 & 1.36300000\end{array}$

$\begin{array}{lllll}\mathrm{Cl} & & -14.82947800 & 4.78908600 & -1.36300000\end{array}$

$\begin{array}{lllll}\mathrm{Mg} & -16.93147800 & 8.43008600 & 0.00000000\end{array}$

$\begin{array}{lllll}\mathrm{Cl} & -14.82947800 & 8.43008600 & -1.36300000\end{array}$

$\mathrm{Cl} \quad-12.72747800 \quad-13.41691400 \quad 1.36300000$

$\begin{array}{llll}\mathrm{Mg} & -13.77847800 & -11.59591400 & 0.00000000\end{array}$

$\begin{array}{lllll}\mathrm{Cl} & & -12.72747800 & -9.77491400 & 1.36300000\end{array}$

$\mathrm{Cl} \quad-11.67547800 \quad-11.59591400 \quad-1.36300000$

$\begin{array}{lllll}\mathrm{Mg} & -13.77847800 & -7.95491400 & 0.00000000\end{array}$

$\mathrm{Cl} \quad \quad-12.72747800 \quad-6.13391400 \quad 1.36300000$

$\mathrm{Cl} \quad \begin{array}{llll}\mathrm{Cl} & -11.67547800 & -7.95491400 & -1.36300000\end{array}$

$\begin{array}{llll}\mathrm{Mg} & -13.77847800 & -4.31391400 & 0.00000000\end{array}$

$\begin{array}{llll}\mathrm{Cl} & -12.72747800 & -2.49291400 & 1.36300000\end{array}$

$\mathrm{Cl} \quad-11.67547800 \quad-4.31391400 \quad-1.36300000$

$\begin{array}{lllll}\mathrm{Mg} & -13.77847800 & -0.67291400 & 0.00000000\end{array}$

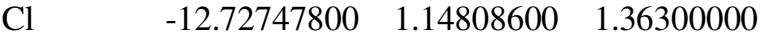

$\begin{array}{lllll}\mathrm{Cl} & & -11.67547800 & -0.67291400 & -1.36300000\end{array}$

$\begin{array}{llll}\mathrm{Mg} & -13.77847800 & 2.96808600 & 0.00000000\end{array}$

$\begin{array}{llll}\mathrm{Cl} & -12.72747800 & 4.78908600 & 1.36300000\end{array}$

$\begin{array}{llll}\mathrm{Cl} & -11.67547800 & 2.96808600 & -1.36300000\end{array}$

$\begin{array}{lllll}\mathrm{Mg} & -13.77847800 & 6.60908600 & 0.00000000\end{array}$

$\begin{array}{lllll}\mathrm{Cl} & -11.67547800 & 6.60908600 & -1.36300000\end{array}$

$\mathrm{Cl} \quad-9.57347800 \quad-15.23691400 \quad 1.36300000$

$\begin{array}{llll}\mathrm{Mg} & -10.62447800 & -13.41691400 & 0.00000000\end{array}$

$\mathrm{Cl} \quad-9.57347800 \quad-11.59591400 \quad 1.36300000$

$\mathrm{Cl} \quad-8.52247800 \quad-13.41691400 \quad-1.36300000$

$\begin{array}{lllll}\mathrm{Mg} & -10.62447800 & -9.77491400 & 0.00000000\end{array}$

$\begin{array}{lllll}\mathrm{Cl} & & -9.57347800 & -7.95491400 & 1.36300000\end{array}$

$\mathrm{Cl} \quad-8.52247800 \quad-9.77491400 \quad-1.36300000$

$\begin{array}{llll}\mathrm{Mg} & -10.62447800 & -6.13391400 & 0.00000000\end{array}$

$\mathrm{Cl} \quad-9.57347800 \quad-4.31391400 \quad 1.36300000$

$\mathrm{Cl} \quad-8.52247800 \quad-6.13391400 \quad-1.36300000$

$\begin{array}{lllll}\mathrm{Mg} & -10.62447800 & -2.49291400 & 0.00000000\end{array}$

$\begin{array}{llll}\mathrm{Cl} & -9.57347800 & -0.67291400 & 1.36300000\end{array}$

$\mathrm{Cl} \quad-8.52247800 \quad-2.49291400 \quad-1.36300000$

$\begin{array}{llll}\mathrm{Mg} & -10.62447800 & 1.14808600 & 0.00000000\end{array}$

$\begin{array}{llll}\mathrm{Cl} & -9.57347800 & 2.96808600 & 1.36300000\end{array}$ 


\begin{tabular}{|c|c|}
\hline $\mathrm{Cl}$ & $\begin{array}{lll}-8.52247800 & 1.14808600 & -1.36300000\end{array}$ \\
\hline $\mathrm{Mg}$ & $\begin{array}{lll}-10.62447800 & 4.78908600 & 0.00000000\end{array}$ \\
\hline $\mathrm{Cl}$ & $\begin{array}{lll}-8.52247800 & 4.78908600 & -1.36300000\end{array}$ \\
\hline $\mathrm{Cl}$ & $\begin{array}{lll}-6.42047800 & -17.05791400 & 1.36300000\end{array}$ \\
\hline $\mathrm{Mg}$ & $-7.47147800 \quad-15.23691400 \quad 0.00000000$ \\
\hline $\mathrm{Cl}$ & $\begin{array}{lll}-6.42047800 & -13.41691400 & 1.36300000\end{array}$ \\
\hline $\mathrm{Cl}$ & $\begin{array}{lll}-5.36947800 & -15.23691400 & -1.36300000\end{array}$ \\
\hline $\mathrm{Mg}$ & $-7.47147800-11.59591400 \quad 0.00000000$ \\
\hline $\mathrm{Cl}$ & $\begin{array}{lll}-6.42047800 & -9.77491400 & 1.36300000\end{array}$ \\
\hline $\mathrm{Cl}$ & $\begin{array}{lll}-5.36947800 & -11.59591400 & -1.36300000\end{array}$ \\
\hline $\mathrm{Mg}$ & $\begin{array}{lll}-7.47147800 & -7.95491400 & 0.00000000\end{array}$ \\
\hline $\mathrm{Cl}$ & $-6.42047800 \quad-6.13391400 \quad 1.36300000$ \\
\hline $\mathrm{Cl}$ & $\begin{array}{lll}-5.36947800 & -7.95491400 & -1.36300000\end{array}$ \\
\hline $\mathrm{Mg}$ & $-7.47147800 \quad-4.31391400 \quad 0.00000000$ \\
\hline $\mathrm{Cl}$ & $\begin{array}{lll}-6.42047800 & -2.49291400 & 1.36300000\end{array}$ \\
\hline $\mathrm{Cl}$ & $\begin{array}{lll}-5.36947800 & -4.31391400 & -1.36300000\end{array}$ \\
\hline $\mathrm{Mg}$ & $-7.47147800 \quad-0.67291400 \quad 0.00000000$ \\
\hline $\mathrm{Cl}$ & $\begin{array}{lll}-6.42047800 & 1.14808600 & 1.36300000\end{array}$ \\
\hline $\mathrm{Cl}$ & $\begin{array}{lll}-5.36947800 & -0.67291400 & -1.36300000\end{array}$ \\
\hline $\mathrm{Mg}$ & $\begin{array}{lll}-7.47147800 & 2.96808600 & 0.00000000\end{array}$ \\
\hline $\mathrm{Cl}$ & $\begin{array}{lll}-5.36947800 & 2.96808600 & -1.36300000\end{array}$ \\
\hline $\mathrm{Cl}$ & $\begin{array}{lll}-3.26747800 & -18.87791400 & 1.36300000\end{array}$ \\
\hline $\mathrm{Mg}$ & $-4.31847800-17.05791400 \quad 0.00000000$ \\
\hline $\mathrm{Cl}$ & $\begin{array}{lll}-3.26747800 & -15.23691400 & 1.36300000\end{array}$ \\
\hline $\mathrm{Cl}$ & $\begin{array}{lll}-2.21647800 & -17.05791400 & -1.36300000\end{array}$ \\
\hline $\mathrm{Mg}$ & $-4.31847800-13.41691400 \quad 0.00000000$ \\
\hline $\mathrm{Cl}$ & $\begin{array}{lll}-3.26747800 & -11.59591400 & 1.36300000\end{array}$ \\
\hline $\mathrm{Cl}$ & $\begin{array}{lll}-2.21647800 & -13.41691400 & -1.36300000\end{array}$ \\
\hline $\mathrm{Mg}$ & $-4.31847800 \quad-9.77491400 \quad 0.00000000$ \\
\hline $\mathrm{Cl}$ & $\begin{array}{lll}-3.26747800 & -7.95491400 & 1.36300000\end{array}$ \\
\hline $\mathrm{Cl}$ & $\begin{array}{lll}-2.21647800 & -9.77491400 & -1.36300000\end{array}$ \\
\hline $\mathrm{Mg}$ & $-4.31847800 \quad-6.13391400 \quad 0.00000000$ \\
\hline $\mathrm{Cl}$ & $-3.26747800 \quad-4.31391400 \quad 1.36300000$ \\
\hline $\mathrm{Cl}$ & $-2.21647800 \quad-6.13391400 \quad-1.36300000$ \\
\hline $\mathrm{Mg}$ & $-4.31847800 \quad-2.49291400 \quad 0.00000000$ \\
\hline $\mathrm{Cl}$ & $\begin{array}{lll}-3.26747800 & -0.67291400 & 1.36300000\end{array}$ \\
\hline $\mathrm{Cl}$ & $-2.21647800 \quad-2.49291400 \quad-1.36300000$ \\
\hline $\mathrm{Mg}$ & $-4.31847800 \quad 1.14808600 \quad 0.00000000$ \\
\hline $\mathrm{Cl}$ & $\begin{array}{lll}-2.21647800 & 1.14808600 & -1.36300000\end{array}$ \\
\hline $\mathrm{Cl}$ & $\begin{array}{lll}-0.11447800 & -20.69891400 & 1.36300000\end{array}$ \\
\hline $\mathrm{Mg}$ & $\begin{array}{lll}-1.16547800 & -18.87791400 & 0.00000000\end{array}$ \\
\hline $\mathrm{Cl}$ & $\begin{array}{lll}-0.11447800 & -17.05791400 & 1.36300000\end{array}$ \\
\hline $\mathrm{Mg}$ & $\begin{array}{lll}-1.16547800 & -15.23691400 & 0.00000000\end{array}$ \\
\hline $\mathrm{Cl}$ & $\begin{array}{lll}-0.11447800 & -13.41691400 & 1.36300000\end{array}$ \\
\hline $\mathrm{Mg}$ & $\begin{array}{lll}-1.16547800 & -11.59591400 & 0.00000000\end{array}$ \\
\hline $\mathrm{Cl}$ & $-0.11447800 \quad-9.77491400 \quad 1.36300000$ \\
\hline $\mathrm{Mg}$ & $-1.16547800 \quad-7.95491400 \quad 0.00000000$ \\
\hline $\mathrm{Cl}$ & $-0.11447800 \quad-6.13391400 \quad 1.36300000$ \\
\hline $\mathrm{Mg}$ & $-1.16547800 \quad-4.31391400 \quad 0.00000000$ \\
\hline $\mathrm{Cl}$ & $-0.11447800 \quad-2.49291400 \quad 1.36300000$ \\
\hline $\mathrm{Mg}$ & $-1.16547800 \quad-0.67291400 \quad 0.00000000$ \\
\hline
\end{tabular}

\section{$\mathrm{Mg}_{49} \mathrm{Cl}_{98}$ (Optimized)}

Input Keywords:

\#p opt hf/gen geom=connectivity scfcyc=800

Input coordinates:

$\begin{array}{llrc}\mathrm{Cl} & 0.85467100 & 2.10907200 & 11.35273100 \\ \mathrm{Cl} & 1.44337900 & -1.07352400 & 9.13782600 \\ \mathrm{Cl} & 1.32840100 & -4.28447200 & 7.45682700\end{array}$




\begin{tabular}{|c|c|c|c|}
\hline $\mathrm{Cl}$ & 1.30574500 & -7.45796800 & 5.62854300 \\
\hline $\mathrm{Cl}$ & 1.35556700 & -10.59975900 & 3.77006100 \\
\hline $\mathrm{Cl}$ & 1.48893600 & -13.69514500 & 1.90115100 \\
\hline $\mathrm{Cl}$ & 1.78015800 & -16.65048200 & 0.00000000 \\
\hline $\mathrm{Cl}$ & 1.00394700 & 5.43169100 & 9.52999000 \\
\hline $\mathrm{Cl}$ & 1.41863400 & 2.12527300 & 7.43134600 \\
\hline $\mathrm{Cl}$ & 1.35647900 & -1.08906300 & 5.53817700 \\
\hline $\mathrm{Cl}$ & 1.32514900 & -4.28533200 & 3.73172000 \\
\hline $\mathrm{Cl}$ & 1.32475200 & -7.44900900 & 1.87895900 \\
\hline $\mathrm{Cl}$ & 1.37978200 & -10.58780700 & 0.00000000 \\
\hline $\mathrm{Cl}$ & 1.48893600 & -13.69514500 & -1.90115100 \\
\hline $\mathrm{Cl}$ & 1.03420600 & 8.65987800 & 7.66043900 \\
\hline $\mathrm{Cl}$ & 1.45695300 & 5.32163800 & 5.59723000 \\
\hline $\mathrm{Cl}$ & 1.38813500 & 2.12681000 & 3.72316400 \\
\hline $\mathrm{Cl}$ & 1.35537500 & -1.08854400 & 1.85067800 \\
\hline $\mathrm{Cl}$ & 1.32208500 & -4.27801800 & 0.00000000 \\
\hline $\mathrm{Cl}$ & 1.32475200 & -7.44900900 & -1.87895900 \\
\hline $\mathrm{Cl}$ & 1.35556700 & -10.59975900 & -3.77006100 \\
\hline $\mathrm{Cl}$ & 0.97255200 & 11.86229800 & 5.78465800 \\
\hline $\mathrm{Cl}$ & 1.45055900 & 8.52228900 & 3.73294100 \\
\hline $\mathrm{Cl}$ & 1.41034900 & 5.33236600 & 1.87419300 \\
\hline $\mathrm{Cl}$ & 1.39558500 & 2.12098200 & 0.00000000 \\
\hline $\mathrm{Cl}$ & 1.35537500 & -1.08854400 & -1.85067800 \\
\hline $\mathrm{Cl}$ & 1.32514900 & -4.28533200 & -3.73172000 \\
\hline $\mathrm{Cl}$ & 1.30574500 & -7.45796800 & -5.62854300 \\
\hline $\mathrm{Cl}$ & 0.78553100 & 15.05088700 & 3.91223000 \\
\hline $\mathrm{Cl}$ & 1.37120900 & 11.71650100 & 1.85760300 \\
\hline $\mathrm{Cl}$ & 1.38910500 & 8.53289000 & 0.00000000 \\
\hline $\mathrm{Cl}$ & 1.41034900 & 5.33236600 & -1.87419300 \\
\hline $\mathrm{Cl}$ & 1.38813500 & 2.12681000 & -3.72316400 \\
\hline $\mathrm{Cl}$ & 1.35647900 & -1.08906300 & -5.53817700 \\
\hline $\mathrm{Cl}$ & 1.32840100 & -4.28447200 & -7.45682700 \\
\hline $\mathrm{Cl}$ & 0.56900500 & 18.17611200 & 1.96560500 \\
\hline $\mathrm{Cl}$ & 1.25489500 & 14.88147600 & 0.00000000 \\
\hline $\mathrm{Cl}$ & 1.37120900 & 11.71650100 & -1.85760300 \\
\hline $\mathrm{Cl}$ & 1.45055900 & 8.52228900 & -3.73294100 \\
\hline $\mathrm{Cl}$ & 1.45695300 & 5.32163800 & -5.59723000 \\
\hline $\mathrm{Cl}$ & 1.41863400 & 2.12527300 & -7.43134600 \\
\hline $\mathrm{Cl}$ & 1.44337900 & -1.07352400 & -9.13782600 \\
\hline $\mathrm{Cl}$ & -1.83640800 & 20.75969600 & 0.00000000 \\
\hline $\mathrm{Cl}$ & 0.56900500 & 18.17611200 & -1.96560500 \\
\hline $\mathrm{Cl}$ & 0.78553100 & 15.05088700 & -3.91223000 \\
\hline $\mathrm{Cl}$ & 0.97255200 & 11.86229800 & -5.78465800 \\
\hline $\mathrm{Cl}$ & 1.03420600 & 8.65987800 & -7.66043900 \\
\hline $\mathrm{Cl}$ & 1.00394700 & 5.43169100 & -9.52999000 \\
\hline $\mathrm{Cl}$ & 0.85467100 & $2.10907200-$ & -11.35273100 \\
\hline $\mathrm{Mg}$ & 0.00000000 & 0.00000000 & 10.76208500 \\
\hline $\mathrm{Mg}$ & -0.15691700 & $\begin{array}{ll}0 & -3.21894300\end{array}$ & 9.25281000 \\
\hline $\mathrm{Mg}$ & -0.21985000 & $0 \quad-6.41956600$ & 7.44869900 \\
\hline $\mathrm{Mg}$ & -0.20327300 & $\begin{array}{ll}0 & -9.60597200\end{array}$ & 5.59211100 \\
\hline $\mathrm{Mg}$ & -0.10054400 & $0-12.77335600$ & 3.72134900 \\
\hline $\mathrm{Mg}$ & 0.11955200 & -15.91123100 & 1.83832700 \\
\hline $\mathrm{Mg}$ & 0.62730300 & -18.90013500 & 0.00000000 \\
\hline $\mathrm{Mg}$ & 0.15691700 & 3.21894300 & 9.25281000 \\
\hline $\mathrm{Mg}$ & 0.00000000 & 0.00000000 & 7.32046200 \\
\hline $\mathrm{Mg}$ & -0.01584600 & $0-3.23207800$ & 5.57156700 \\
\hline $\mathrm{Mg}$ & -0.03227600 & $\begin{array}{ll}0 & -6.42710900\end{array}$ & 3.74354400 \\
\hline $\mathrm{Mg}$ & 0.00037500 & -9.60548600 & 1.88157300 \\
\hline $\mathrm{Mg}$ & 0.12944300 & -12.77119400 & 0.00000000 \\
\hline $\mathrm{Mg}$ & 0.11955200 & -15.91123100 & -1.83832700 \\
\hline $\mathrm{Mg}$ & 0.21985000 & 6.41956600 & 7.44869900 \\
\hline
\end{tabular}




\begin{tabular}{|c|c|c|c|}
\hline $\mathrm{Mg}$ & 0.01584600 & 3.23207800 & 5.57156700 \\
\hline $\mathrm{Mg}$ & 0.00000000 & 0.00000000 & 3.67678800 \\
\hline $\mathrm{Mg}$ & -0.04397600 & -3.21853800 & 1.85614500 \\
\hline $\mathrm{Mg}$ & -0.05267800 & -6.41637900 & 0.00000000 \\
\hline $\mathrm{Mg}$ & 0.00037500 & -9.60548600 & -1.88157300 \\
\hline $\mathrm{Mg}$ & -0.10054400 & -12.77335600 & -3.72134900 \\
\hline $\mathrm{Mg}$ & 0.20327300 & 9.60597200 & 5.59211100 \\
\hline $\mathrm{Mg}$ & 0.03227600 & 6.42710900 & 3.74354400 \\
\hline $\mathrm{Mg}$ & 0.04397600 & 3.21853800 & 1.85614500 \\
\hline $\mathrm{Mg}$ & 0.00000000 & 0.00000000 & 0.00000000 \\
\hline $\mathrm{Mg}$ & -0.04397600 & -3.21853800 & -1.85614500 \\
\hline $\mathrm{Mg}$ & -0.03227600 & -6.42710900 & -3.74354400 \\
\hline $\mathrm{Mg}$ & -0.20327300 & -9.60597200 & -5.59211100 \\
\hline $\mathrm{Mg}$ & 0.10054400 & 12.77335600 & 3.72134900 \\
\hline $\mathrm{Mg}$ & -0.00037500 & 9.60548600 & 1.88157300 \\
\hline $\mathrm{Mg}$ & 0.05267800 & 6.41637900 & 0.00000000 \\
\hline $\mathrm{Mg}$ & 0.04397600 & 3.21853800 & -1.85614500 \\
\hline $\mathrm{Mg}$ & 0.00000000 & 0.00000000 & -3.67678800 \\
\hline $\mathrm{Mg}$ & -0.01584600 & -3.23207800 & -5.57156700 \\
\hline $\mathrm{Mg}$ & -0.21985000 & -6.41956600 & -7.44869900 \\
\hline $\mathrm{Mg}$ & -0.11955200 & 15.91123100 & 1.83832700 \\
\hline $\mathrm{Mg}$ & -0.12944300 & 12.77119400 & 0.00000000 \\
\hline $\mathrm{Mg}$ & -0.00037500 & 9.60548600 & -1.88157300 \\
\hline $\mathrm{Mg}$ & 0.03227600 & 6.42710900 & -3.74354400 \\
\hline $\mathrm{Mg}$ & 0.01584600 & 3.23207800 & -5.57156700 \\
\hline $\mathrm{Mg}$ & 0.00000000 & 0.00000000 & -7.32046200 \\
\hline $\mathrm{Mg}$ & -0.15691700 & -3.21894300 & -9.25281000 \\
\hline $\mathrm{Mg}$ & -0.62730300 & 18.90013500 & 0.00000000 \\
\hline $\mathrm{Mg}$ & -0.11955200 & 15.91123100 & -1.83832700 \\
\hline $\mathrm{Mg}$ & 0.10054400 & 12.77335600 & -3.72134900 \\
\hline $\mathrm{Mg}$ & 0.20327300 & 9.60597200 & -5.59211100 \\
\hline $\mathrm{Mg}$ & 0.21985000 & 6.41956600 & -7.44869900 \\
\hline $\mathrm{Mg}$ & 0.15691700 & 3.21894300 & -9.25281000 \\
\hline $\mathrm{Mg}$ & 0.00000000 & 0.00000000 & -10.76208500 \\
\hline $\mathrm{Cl}$ & -0.85467100 & -2.10907200 & 11.35273100 \\
\hline $\mathrm{Cl}$ & -1.00394700 & -5.43169100 & 9.52999000 \\
\hline $\mathrm{Cl}$ & -1.03420600 & -8.65987800 & 7.66043900 \\
\hline $\mathrm{Cl}$ & -0.97255200 & -11.86229800 & 5.78465800 \\
\hline $\mathrm{Cl}$ & -0.78553100 & -15.05088700 & 3.91223000 \\
\hline $\mathrm{Cl}$ & -0.56900500 & -18.17611200 & 1.96560500 \\
\hline $\mathrm{Cl}$ & 1.83640800 & -20.75969600 & 0.00000000 \\
\hline $\mathrm{Cl}$ & -1.44337900 & 1.07352400 & 9.13782600 \\
\hline $\mathrm{Cl}$ & -1.41863400 & -2.12527300 & 7.43134600 \\
\hline $\mathrm{Cl}$ & -1.45695300 & -5.32163800 & 5.59723000 \\
\hline $\mathrm{Cl}$ & -1.45055900 & -8.52228900 & 3.73294100 \\
\hline $\mathrm{Cl}$ & -1.37120900 & -11.71650100 & 1.85760300 \\
\hline $\mathrm{Cl}$ & -1.25489500 & -14.88147600 & 0.00000000 \\
\hline $\mathrm{Cl}$ & -0.56900500 & -18.17611200 & -1.96560500 \\
\hline $\mathrm{Cl}$ & -1.32840100 & 4.28447200 & 7.45682700 \\
\hline $\mathrm{Cl}$ & -1.35647900 & 1.08906300 & 5.53817700 \\
\hline $\mathrm{Cl}$ & -1.38813500 & -2.12681000 & 3.72316400 \\
\hline $\mathrm{Cl}$ & -1.41034900 & -5.33236600 & 1.87419300 \\
\hline $\mathrm{Cl}$ & -1.38910500 & -8.53289000 & 0.00000000 \\
\hline $\mathrm{Cl}$ & -1.37120900 & -11.71650100 & -1.85760300 \\
\hline $\mathrm{Cl}$ & -0.78553100 & -15.05088700 & -3.91223000 \\
\hline $\mathrm{Cl}$ & -1.30574500 & 7.45796800 & 5.62854300 \\
\hline $\mathrm{Cl}$ & -1.32514900 & 4.28533200 & 3.73172000 \\
\hline $\mathrm{Cl}$ & -1.35537500 & 1.08854400 & 1.85067800 \\
\hline $\mathrm{Cl}$ & -1.39558500 & -2.12098200 & 0.00000000 \\
\hline $\mathrm{Cl}$ & -1.41034900 & -5.33236600 & -1.87419300 \\
\hline $\mathrm{Cl}$ & -1.45055900 & -8.52228900 & -3.73294100 \\
\hline
\end{tabular}




$\begin{array}{llcc}\mathrm{Cl} & -0.97255200 & -11.86229800 & -5.78465800 \\ \mathrm{Cl} & -1.35556700 & 10.59975900 & 3.77006100 \\ \mathrm{Cl} & -1.32475200 & 7.44900900 & 1.87895900 \\ \mathrm{Cl} & -1.32208500 & 4.27801800 & 0.00000000 \\ \mathrm{Cl} & -1.35537500 & 1.08854400 & -1.85067800 \\ \mathrm{Cl} & -1.38813500 & -2.12681000 & -3.72316400 \\ \mathrm{Cl} & -1.45695300 & -5.32163800 & -5.59723000 \\ \mathrm{Cl} & -1.03420600 & -8.65987800 & -7.66043900 \\ \mathrm{Cl} & -1.48893600 & 13.69514500 & 1.90115100 \\ \mathrm{Cl} & -1.37978200 & 10.58780700 & 0.00000000 \\ \mathrm{Cl} & -1.32475200 & 7.44900900 & -1.87895900 \\ \mathrm{Cl} & -1.32514900 & 4.28533200 & -3.73172000 \\ \mathrm{Cl} & -1.35647900 & 1.08906300 & -5.53817700 \\ \mathrm{Cl} & -1.41863400 & -2.12527300 & -7.43134600 \\ \mathrm{Cl} & -1.00394700 & -5.43169100 & -9.52999000 \\ \mathrm{Cl} & -1.78015800 & 16.65048200 & 0.00000000 \\ \mathrm{Cl} & -1.48893600 & 13.69514500 & -1.90115100 \\ \mathrm{Cl} & -1.35556700 & 10.59975900 & -3.77006100 \\ \mathrm{Cl} & -1.30574500 & 7.45796800 & -5.62854300 \\ \mathrm{Cl} & -1.32840100 & 4.28447200 & -7.45682700 \\ \mathrm{Cl} & -1.44337900 & 1.07352400 & -9.13782600 \\ \mathrm{Cl} & -0.85467100 & -2.10907200 & -11.35273100\end{array}$

Output coordinates:

\begin{tabular}{|c|c|c|c|}
\hline $\mathrm{Cl}$ & 0.85043800 & 2.11077400 & 9.50106400 \\
\hline $\mathrm{Cl}$ & 1.44737800 & -1.06761700 & 7.28832800 \\
\hline $\mathrm{Cl}$ & 1.34480600 & -4.26675400 & 5.60272100 \\
\hline $\mathrm{Cl}$ & 1.34646100 & -7.41555000 & 3.76919500 \\
\hline $\mathrm{Cl}$ & 1.43835600 & -10.51557100 & 1.90387300 \\
\hline $\mathrm{Cl}$ & 1.68652500 & -13.47497800 & 0.00000000 \\
\hline $\mathrm{Cl}$ & 0.98593300 & 5.42856400 & 7.67090400 \\
\hline $\mathrm{Cl}$ & 1.41294900 & 2.12481900 & 5.57761000 \\
\hline $\mathrm{Cl}$ & 1.36096500 & -1.08196100 & 3.68566700 \\
\hline $\mathrm{Cl}$ & 1.34229700 & -4.26518600 & 1.86914700 \\
\hline $\mathrm{Cl}$ & 1.36576800 & -7.40853700 & 0.00000000 \\
\hline $\mathrm{Cl}$ & 1.43835600 & -10.51557100 & -1.90387300 \\
\hline $\mathrm{Cl}$ & 0.99090900 & 8.64703100 & 5.79180100 \\
\hline $\mathrm{Cl}$ & 1.43684900 & 5.31231700 & 3.73508300 \\
\hline $\mathrm{Cl}$ & 1.38318700 & 2.12590700 & 1.86475200 \\
\hline $\mathrm{Cl}$ & 1.36138100 & -1.08245500 & 0.00000000 \\
\hline $\mathrm{Cl}$ & 1.34229700 & -4.26518600 & -1.86914700 \\
\hline $\mathrm{Cl}$ & 1.34646100 & -7.41555000 & -3.76919500 \\
\hline $\mathrm{Cl}$ & 0.85293600 & 11.84139700 & 3.91508400 \\
\hline $\mathrm{Cl}$ & 1.39616600 & 8.50372200 & 1.86126400 \\
\hline $\mathrm{Cl}$ & 1.38432300 & 5.32682600 & 0.00000000 \\
\hline $\mathrm{Cl}$ & 1.38318700 & 2.12590700 & -1.86475200 \\
\hline $\mathrm{Cl}$ & 1.36096500 & -1.08196100 & -3.68566700 \\
\hline $\mathrm{Cl}$ & 1.34480600 & -4.26675400 & -5.60272100 \\
\hline $\mathrm{Cl}$ & 0.68272200 & 14.96937500 & 1.96507900 \\
\hline $\mathrm{Cl}$ & 1.31992900 & 11.66455300 & 0.00000000 \\
\hline $\mathrm{Cl}$ & 1.39616600 & 8.50372200 & -1.86126400 \\
\hline $\mathrm{Cl}$ & 1.43684900 & 5.31231700 & -3.73508300 \\
\hline $\mathrm{Cl}$ & 1.41294900 & 2.12481900 & -5.57761000 \\
\hline $\mathrm{Cl}$ & 1.44737800 & -1.06761700 & -7.28832800 \\
\hline $\mathrm{Cl}$ & -1.69155000 & 17.58377800 & 0.00000000 \\
\hline $\mathrm{Cl}$ & 0.68272200 & 14.96937500 & -1.96507900 \\
\hline $\mathrm{Cl}$ & 0.85293600 & 11.84139700 & -3.91508400 \\
\hline $\mathrm{Cl}$ & 0.99090900 & 8.64703100 & -5.79180100 \\
\hline $\mathrm{Cl}$ & 0.98593300 & 5.42856400 & -7.67090400 \\
\hline $\mathrm{Cl}$ & 0.85043800 & 2.11077400 & -9.50106400 \\
\hline $\mathrm{Mg}$ & 0.00000000 & 0.00000000 & 8.91373700 \\
\hline $\mathrm{Mg}$ & -0.14642100 & -3.21485100 & 7.39992200 \\
\hline
\end{tabular}




\begin{tabular}{|c|c|c|c|}
\hline $\mathrm{Mg}$ & -0.19172400 & -6.40541900 & 5.58761900 \\
\hline $\mathrm{Mg}$ & -0.13713400 & -9.57515000 & 3.72375100 \\
\hline $\mathrm{Mg}$ & 0.03789500 & -12.71624100 & 1.83987800 \\
\hline $\mathrm{Mg}$ & 0.50378800 & -15.71124400 & 0.00000000 \\
\hline $\mathrm{Mg}$ & 0.14642100 & 3.21485100 & 7.39992200 \\
\hline $\mathrm{Mg}$ & 0.00000000 & 0.00000000 & 5.47211700 \\
\hline $\mathrm{Mg}$ & -0.00421400 & -3.22583200 & 3.71678400 \\
\hline $\mathrm{Mg}$ & -0.00448600 & -6.41118700 & 1.87845400 \\
\hline $\mathrm{Mg}$ & 0.09176100 & -9.57891700 & 0.00000000 \\
\hline $\mathrm{Mg}$ & 0.03789500 & -12.71624100 & -1.83987800 \\
\hline $\mathrm{Mg}$ & 0.19172400 & 6.40541900 & 5.58761900 \\
\hline $\mathrm{Mg}$ & 0.00421400 & 3.22583200 & 3.71678400 \\
\hline $\mathrm{Mg}$ & 0.00000000 & 0.00000000 & 1.83098200 \\
\hline $\mathrm{Mg}$ & -0.03564600 & -3.21440600 & 0.00000000 \\
\hline $\mathrm{Mg}$ & -0.00448600 & -6.41118700 & -1.87845400 \\
\hline $\mathrm{Mg}$ & -0.13713400 & -9.57515000 & -3.72375100 \\
\hline $\mathrm{Mg}$ & 0.13713400 & 9.57515000 & 3.72375100 \\
\hline $\mathrm{Mg}$ & 0.00448600 & 6.41118700 & 1.87845400 \\
\hline $\mathrm{Mg}$ & 0.03564600 & 3.21440600 & 0.00000000 \\
\hline $\mathrm{Mg}$ & 0.00000000 & 0.00000000 & -1.83098200 \\
\hline $\mathrm{Mg}$ & -0.00421400 & -3.22583200 & -3.71678400 \\
\hline $\mathrm{Mg}$ & -0.19172400 & -6.40541900 & -5.58761900 \\
\hline $\mathrm{Mg}$ & -0.03789500 & 12.71624100 & 1.83987800 \\
\hline $\mathrm{Mg}$ & -0.09176100 & 9.57891700 & 0.00000000 \\
\hline $\mathrm{Mg}$ & 0.00448600 & 6.41118700 & -1.87845400 \\
\hline $\mathrm{Mg}$ & 0.00421400 & 3.22583200 & -3.71678400 \\
\hline $\mathrm{Mg}$ & 0.00000000 & 0.00000000 & -5.47211700 \\
\hline $\mathrm{Mg}$ & -0.14642100 & -3.21485100 & -7.39992200 \\
\hline $\mathrm{Mg}$ & -0.50378800 & 15.71124400 & 0.00000000 \\
\hline $\mathrm{Mg}$ & -0.03789500 & 12.71624100 & -1.83987800 \\
\hline $\mathrm{Mg}$ & 0.13713400 & 9.57515000 & -3.72375100 \\
\hline $\mathrm{Mg}$ & 0.19172400 & 6.40541900 & -5.58761900 \\
\hline $\mathrm{Mg}$ & 0.14642100 & 3.21485100 & -7.39992200 \\
\hline $\mathrm{Mg}$ & 0.00000000 & 0.00000000 & -8.91373700 \\
\hline $\mathrm{Cl}$ & -0.85043800 & -2.11077400 & 9.50106400 \\
\hline $\mathrm{Cl}$ & -0.98593300 & -5.42856400 & 7.67090400 \\
\hline $\mathrm{Cl}$ & -0.99090900 & -8.64703100 & 5.79180100 \\
\hline $\mathrm{Cl}$ & -0.85293600 & -11.84139700 & 3.91508400 \\
\hline $\mathrm{Cl}$ & -0.68272200 & -14.96937500 & 1.96507900 \\
\hline $\mathrm{Cl}$ & 1.69155000 & -17.58377800 & 0.00000000 \\
\hline $\mathrm{Cl}$ & -1.44737800 & 1.06761700 & 7.28832800 \\
\hline $\mathrm{Cl}$ & -1.41294900 & -2.12481900 & 5.57761000 \\
\hline $\mathrm{Cl}$ & -1.43684900 & -5.31231700 & 3.73508300 \\
\hline $\mathrm{Cl}$ & -1.39616600 & -8.50372200 & 1.86126400 \\
\hline $\mathrm{Cl}$ & -1.31992900 & -11.66455300 & 0.00000000 \\
\hline $\mathrm{Cl}$ & -0.68272200 & -14.96937500 & -1.96507900 \\
\hline $\mathrm{Cl}$ & -1.34480600 & 4.26675400 & 5.60272100 \\
\hline $\mathrm{Cl}$ & -1.36096500 & 1.08196100 & 3.68566700 \\
\hline $\mathrm{Cl}$ & -1.38318700 & -2.12590700 & 1.86475200 \\
\hline $\mathrm{Cl}$ & -1.38432300 & -5.32682600 & 0.00000000 \\
\hline $\mathrm{Cl}$ & -1.39616600 & -8.50372200 & -1.86126400 \\
\hline $\mathrm{Cl}$ & -0.85293600 & -11.84139700 & -3.91508400 \\
\hline $\mathrm{Cl}$ & -1.34646100 & 7.41555000 & 3.76919500 \\
\hline $\mathrm{Cl}$ & -1.34229700 & 4.26518600 & 1.86914700 \\
\hline $\mathrm{Cl}$ & -1.36138100 & 1.08245500 & 0.00000000 \\
\hline $\mathrm{Cl}$ & -1.38318700 & -2.12590700 & -1.86475200 \\
\hline $\mathrm{Cl}$ & -1.43684900 & -5.31231700 & -3.73508300 \\
\hline $\mathrm{Cl}$ & -0.99090900 & -8.64703100 & -5.79180100 \\
\hline $\mathrm{Cl}$ & -1.43835600 & 10.51557100 & 1.90387300 \\
\hline $\mathrm{Cl}$ & -1.36576800 & 7.40853700 & 0.00000000 \\
\hline $\mathrm{Cl}$ & -1.34229700 & 4.26518600 & -1.86914700 \\
\hline
\end{tabular}




\begin{tabular}{|c|c|c|c|}
\hline $\mathrm{Cl}$ & -1.36096500 & 1.08196100 & -3.68566700 \\
\hline $\mathrm{Cl}$ & -1.41294900 & -2.12481900 & -5.57761000 \\
\hline $\mathrm{Cl}$ & -0.98593300 & -5.42856400 & -7.67090400 \\
\hline $\mathrm{Cl}$ & -1.68652500 & 13.47497800 & 0.00000000 \\
\hline $\mathrm{Cl}$ & -1.43835600 & 10.51557100 & -1.90387300 \\
\hline $\mathrm{Cl}$ & -1.34646100 & 7.41555000 & -3.76919500 \\
\hline $\mathrm{Cl}$ & -1.34480600 & 4.26675400 & -5.60272100 \\
\hline $\mathrm{Cl}$ & -1.44737800 & 1.06761700 & -7.28832800 \\
\hline $\mathrm{Cl}$ & -0.85043800 & -2.11077400 & -9.50106400 \\
\hline
\end{tabular}

\section{$\mathrm{Mg}_{49} \mathrm{Cl}_{98}$ (Frozen)}

Input Keywords:

$\# p$ hf/Gen geom=connectivity scfcyc $=800$

Input coordinates:

$\begin{array}{lccc}\mathrm{Cl} & -17.98247800 & -7.95491400 & -1.36300000 \\ \mathrm{Cl} & -17.98247800 & -4.31391400 & -1.36300000 \\ \mathrm{Cl} & -17.98247800 & -0.67291400 & -1.36300000 \\ \mathrm{Cl} & -17.98247800 & 2.96808600 & -1.36300000 \\ \mathrm{Cl} & -17.98247800 & 6.60908600 & -1.36300000 \\ \mathrm{Cl} & -17.98247800 & 10.25008600 & -1.36300000 \\ \mathrm{Cl} & -17.98247800 & 13.89108600 & -1.36300000 \\ \mathrm{Cl} & -15.88047800 & -11.59591400 & 1.36300000 \\ \mathrm{Mg} & -16.93147800 & -9.77491400 & 0.00000000 \\ \mathrm{Cl} & -15.88047800 & -7.95491400 & 1.36300000 \\ \mathrm{Cl} & -14.82947800 & -9.77491400 & -1.36300000 \\ \mathrm{Mg} & -16.93147800 & -6.13391400 & 0.00000000 \\ \mathrm{Cl} & -15.88047800 & -4.31391400 & 1.36300000 \\ \mathrm{Cl} & -14.82947800 & -6.13391400 & -1.36300000 \\ \mathrm{Mg} & -16.93147800 & -2.49291400 & 0.00000000 \\ \mathrm{Cl} & -15.88047800 & -0.67291400 & 1.36300000 \\ \mathrm{Cl} & -14.82947800 & -2.49291400 & -1.36300000 \\ \mathrm{Mg} & -16.93147800 & 1.14808600 & 0.00000000 \\ \mathrm{Cl} & -15.88047800 & 2.96808600 & 1.36300000 \\ \mathrm{Cl} & -14.82947800 & 1.14808600 & -1.36300000 \\ \mathrm{Mg} & -16.93147800 & 4.78908600 & 0.00000000 \\ \mathrm{Cl} & -15.88047800 & 6.60908600 & 1.36300000 \\ \mathrm{Cl} & -14.82947800 & 4.78908600 & -1.36300000 \\ \mathrm{Mg} & -16.93147800 & 8.43008600 & 0.00000000 \\ \mathrm{Cl} & -15.88047800 & 10.25008600 & 1.36300000 \\ \mathrm{Cl} & -14.82947800 & 8.43008600 & -1.36300000 \\ \mathrm{Mg} & -16.93147800 & 12.07108600 & 0.00000000 \\ \mathrm{Cl} & -14.82947800 & 12.07108600 & -1.36300000 \\ \mathrm{Cl} & -12.72747800 & -13.41691400 & 1.36300000 \\ \mathrm{Mg} & -13.77847800 & -11.59591400 & 0.00000000 \\ \mathrm{Cl} & -12.72747800 & -9.77491400 & 1.36300000 \\ \mathrm{Cl} & -11.67547800 & -11.59591400 & -1.36300000 \\ \mathrm{Mg} & -13.77847800 & -7.95491400 & 0.00000000 \\ \mathrm{Cl} & -12.72747800 & -6.13391400 & 1.36300000 \\ \mathrm{Cl} & -11.67547800 & -7.95491400 & -1.36300000 \\ \mathrm{Mg} & -13.77847800 & -4.31391400 & 0.00000000 \\ \mathrm{Cl} & -12.72747800 & -2.49291400 & 1.36300000 \\ \mathrm{Cl} & -11.67547800 & -4.31391400 & -1.36300000 \\ \mathrm{Mg} & -13.77847800 & -0.67291400 & 0.00000000 \\ \mathrm{Cl} & -12.72747800 & 1.14808600 & 1.36300000 \\ \mathrm{Cl} & -11.67547800 & -0.67291400 & -1.36300000 \\ \mathrm{Mg} & -13.77847800 & 2.96808600 & 0.00000000 \\ \mathrm{Cl} & -12.72747800 & 4.78908600 & 1.36300000 \\ \mathrm{Cl} & -11.67547800 & 2.96808600 & -1.36300000 \\ \mathrm{Mg} & -13.77847800 & 6.60908600 & 0.00000000\end{array}$




\begin{tabular}{|c|c|}
\hline $\mathrm{Cl}$ & $-12.72747800 \quad 8.43008600$ \\
\hline $\mathrm{Cl}$ & $\begin{array}{lll}-11.67547800 & 6.60908600 & -1.36300000\end{array}$ \\
\hline $\mathrm{Mg}$ & $\begin{array}{lll}-13.77847800 & 10.25008600 & 0.00000000\end{array}$ \\
\hline $\mathrm{Cl}$ & $\begin{array}{lll}-11.67547800 & 10.25008600 & -1.36300000\end{array}$ \\
\hline $\mathrm{Cl}$ & $\begin{array}{lll}-9.57347800 & -15.23691400 & 1.36300000\end{array}$ \\
\hline $\mathrm{Mg}$ & $\begin{array}{lll}-10.62447800 & -13.41691400 & 0.00000000\end{array}$ \\
\hline $\mathrm{Cl}$ & $\begin{array}{lll}-9.57347800 & -11.59591400 & 1.36300000\end{array}$ \\
\hline $\mathrm{Cl}$ & $\begin{array}{lll}-8.52247800 & -13.41691400 & -1.36300000\end{array}$ \\
\hline $\mathrm{Mg}$ & $\begin{array}{lll}-10.62447800 & -9.77491400 & 0.00000000\end{array}$ \\
\hline $\mathrm{Cl}$ & $\begin{array}{lll}-9.57347800 & -7.95491400 & 1.36300000\end{array}$ \\
\hline $\mathrm{Cl}$ & $\begin{array}{lll}-8.52247800 & -9.77491400 & -1.36300000\end{array}$ \\
\hline $\mathrm{Mg}$ & $-10.62447800-6.13391400 \quad 0.00000000$ \\
\hline $\mathrm{Cl}$ & $\begin{array}{lll}-9.57347800 & -4.31391400 & 1.36300000\end{array}$ \\
\hline $\mathrm{Cl}$ & $\begin{array}{lll}-8.52247800 & -6.13391400 & -1.36300000\end{array}$ \\
\hline $\mathrm{Mg}$ & $-10.62447800-2.49291400 \quad 0.00000000$ \\
\hline $\mathrm{Cl}$ & $\begin{array}{lll}-9.57347800 & -0.67291400 & 1.36300000\end{array}$ \\
\hline $\mathrm{Cl}$ & $\begin{array}{lll}-8.52247800 & -2.49291400 & -1.36300000\end{array}$ \\
\hline $\mathrm{Mg}$ & $-10.62447800 \quad 1.14808600 \quad 0.00000000$ \\
\hline $\mathrm{Cl}$ & $\begin{array}{lll}-9.57347800 & 2.96808600 & 1.36300000\end{array}$ \\
\hline $\mathrm{Cl}$ & $1.14808600-1.363$ \\
\hline $\mathrm{Mg}$ & $\begin{array}{lll}-10.62447800 & 4.78908600 & 0.00000000\end{array}$ \\
\hline $\mathrm{Cl}$ & $\begin{array}{lll}-9.57347800 & 6.60908600 & 1.36300000\end{array}$ \\
\hline $\mathrm{Cl}$ & $4.78908600-1.36300000$ \\
\hline $\mathrm{Mg}$ & $\begin{array}{lll}-10.62447800 & 8.43008600 & 0.00000000\end{array}$ \\
\hline $\mathrm{Cl}$ & $\begin{array}{lll}-8.52247800 & 8.43008600 & -1.36300000\end{array}$ \\
\hline $\mathrm{Cl}$ & $\begin{array}{lll}-6.42047800 & -17.05791400 & 1.36300000\end{array}$ \\
\hline $\mathrm{Mg}$ & $\begin{array}{lll}-7.47147800 & -15.23691400 & 0.0\end{array}$ \\
\hline $\mathrm{Cl}$ & $\begin{array}{lll}-6.42047800 & -13.41691400 & 1.36300000\end{array}$ \\
\hline $\mathrm{Cl}$ & $\begin{array}{lll}-5.36947800 & -15.23691400 & -1.36300000\end{array}$ \\
\hline $\mathrm{Mg}$ & $-7.47147800-11.59591400 \quad 0.00000000$ \\
\hline $\mathrm{Cl}$ & $\begin{array}{lll}-6.42047800 & -9.774491400 & 1.36300000\end{array}$ \\
\hline $\mathrm{Cl}$ & $\begin{array}{lll}-5.36947800 & -11.59591400 & -1.36300000\end{array}$ \\
\hline $\mathrm{Mg}$ & $\begin{array}{lll}-7.47147800 & -7.95491400 & 0.00000000\end{array}$ \\
\hline $\mathrm{Cl}$ & $\begin{array}{lll}-6.42047800 & -6.13391400 & 1.36300000\end{array}$ \\
\hline $\mathrm{Cl}$ & $\begin{array}{lll}-5.36947800 & -7.95491400 & -1.36300000\end{array}$ \\
\hline $\mathrm{Mg}$ & $-7.47147800 \quad-4.31391400 \quad 0.00000000$ \\
\hline $\mathrm{Cl}$ & $\begin{array}{lll}-6.42047800 & -2.49291400 & 1.36300000\end{array}$ \\
\hline $\mathrm{Cl}$ & $\begin{array}{lll}-5.36947800 & -4.31391400 & -1.36300000\end{array}$ \\
\hline $\mathrm{Mg}$ & $-7.47147800 \quad-0.67291400 \quad 0.00000000$ \\
\hline $\mathrm{Cl}$ & $\begin{array}{lll}-6.42047800 & 1.14808600 & 1.36300000\end{array}$ \\
\hline $\mathrm{Cl}$ & $\begin{array}{lll}-5.36947800 & -0.67291400 & -1.36300000\end{array}$ \\
\hline $\mathrm{Mg}$ & $\begin{array}{lll}-7.47147800 & 2.96808600 & 0.00000000\end{array}$ \\
\hline $\mathrm{Cl}$ & $\begin{array}{lll}-6.42047800 & 4.78908600 & 1.363\end{array}$ \\
\hline $\mathrm{Cl}$ & $\begin{array}{lll}-5.36947800 & 2.96808600 & -1.36300000\end{array}$ \\
\hline $\mathrm{Mg}$ & $\begin{array}{lll}-7.47147800 & 6.60908600 & 0.00000000\end{array}$ \\
\hline $\mathrm{Cl}$ & $\begin{array}{lll}-5.36947800 & 6.60908600 & -1.36300000\end{array}$ \\
\hline $\mathrm{Cl}$ & $\begin{array}{lll}-3.26747800 & -18.87791400 & 1.36300000\end{array}$ \\
\hline $\mathrm{Mg}$ & $\begin{array}{lll}-4.31847800 & -17.05791400 & 0.00000000\end{array}$ \\
\hline $\mathrm{Cl}$ & $\begin{array}{lll}-3.26747800 & -15.23691400 & 1.36300000\end{array}$ \\
\hline $\mathrm{Cl}$ & $\begin{array}{lll}-2.21647800 & -17.05791400 & -1.36300000\end{array}$ \\
\hline $\mathrm{Mg}$ & $-4.31847800-13.41691400 \quad 0.00000000$ \\
\hline $\mathrm{Cl}$ & $\begin{array}{lll}-3.26747800 & -11.59591400 & 1.36300000\end{array}$ \\
\hline $\mathrm{Cl}$ & $\begin{array}{lll}-2.21647800 & -13.41691400 & -1.36300000\end{array}$ \\
\hline $\mathrm{Mg}$ & $\begin{array}{lll}-4.31847800 & -9.77491400 & 0.00000000\end{array}$ \\
\hline $\mathrm{Cl}$ & $\begin{array}{lll}-3.26747800 & -7.95491400 & 1.36300000\end{array}$ \\
\hline $\mathrm{Cl}$ & $\begin{array}{lll}-2.21647800 & -9.77491400 & -1.36300000\end{array}$ \\
\hline $\mathrm{Mg}$ & $-4.31847800 \quad-6.13391400 \quad 0.00000000$ \\
\hline $\mathrm{Cl}$ & $-3.26747800 \quad-4.31391400 \quad 1.36300000$ \\
\hline $\mathrm{Cl}$ & $\begin{array}{lll}-2.21647800 & -6.13391400 & -1.36300000\end{array}$ \\
\hline $\mathrm{Mg}$ & $-4.31847800 \quad-2.49291400 \quad 0.00000000$ \\
\hline $\mathrm{Cl}$ & $3.26747800-0.67291400$ \\
\hline
\end{tabular}




\begin{tabular}{lccc}
$\mathrm{Cl}$ & -2.21647800 & -2.49291400 & -1.36300000 \\
$\mathrm{Mg}$ & -4.31847800 & 1.14808600 & 0.00000000 \\
$\mathrm{Cl}$ & -3.26747800 & 2.96808600 & 1.36300000 \\
$\mathrm{Cl}$ & -2.21647800 & 1.14808600 & -1.36300000 \\
$\mathrm{Mg}$ & -4.31847800 & 4.78908600 & 0.00000000 \\
$\mathrm{Cl}$ & -2.21647800 & 4.78908600 & -1.36300000 \\
$\mathrm{Cl}$ & -0.11447800 & -20.69891400 & 1.36300000 \\
$\mathrm{Mg}$ & -1.16547800 & -18.87791400 & 0.00000000 \\
$\mathrm{Cl}$ & -0.11447800 & -17.05791400 & 1.36300000 \\
$\mathrm{Cl}$ & 0.93652200 & -18.87791400 & -1.36300000 \\
$\mathrm{Mg}$ & -1.16547800 & -15.23691400 & 0.00000000 \\
$\mathrm{Cl}$ & -0.11447800 & -13.41691400 & 1.36300000 \\
$\mathrm{Cl}$ & 0.93652200 & -15.23691400 & -1.36300000 \\
$\mathrm{Mg}$ & -1.16547800 & -11.59591400 & 0.00000000 \\
$\mathrm{Cl}$ & -0.11447800 & -9.77491400 & 1.36300000 \\
$\mathrm{Cl}$ & 0.93652200 & -11.59591400 & -1.36300000 \\
$\mathrm{Mg}$ & -1.16547800 & -7.95491400 & 0.00000000 \\
$\mathrm{Cl}$ & -0.11447800 & -6.13391400 & 1.36300000 \\
$\mathrm{Cl}$ & 0.93652200 & -7.95491400 & -1.36300000 \\
$\mathrm{Mg}$ & -1.16547800 & -4.31391400 & 0.00000000 \\
$\mathrm{Cl}$ & -0.11447800 & -2.49291400 & 1.36300000 \\
$\mathrm{Cl}$ & 0.93652200 & -4.31391400 & -1.36300000 \\
$\mathrm{Mg}$ & -1.16547800 & -0.67291400 & 0.00000000 \\
$\mathrm{Cl}$ & -0.11447800 & 1.14808600 & 1.36300000 \\
$\mathrm{Cl}$ & 0.93652200 & -0.67291400 & -1.36300000 \\
$\mathrm{Mg}$ & -1.16547800 & 2.96808600 & 0.00000000 \\
$\mathrm{Cl}$ & 0.93652200 & 2.96808600 & -1.36300000 \\
$\mathrm{Cl}$ & 3.03852200 & -22.51891400 & 1.36300000 \\
$\mathrm{Mg}$ & 1.98752200 & -20.69891400 & 0.00000000 \\
$\mathrm{Cl}$ & 3.03852200 & -18.87791400 & 1.36300000 \\
$\mathrm{Mg}$ & 1.98752200 & -17.05791400 & 0.00000000 \\
$\mathrm{Cl}$ & 3.03852200 & -15.23691400 & 1.36300000 \\
$\mathrm{Mg}$ & 1.98752200 & -13.41691400 & 0.00000000 \\
$\mathrm{Cl}$ & 3.03852200 & -11.59591400 & 1.36300000 \\
$\mathrm{Mg}$ & 1.98752200 & -9.77491400 & 0.00000000 \\
$\mathrm{Cl}$ & 3.03852200 & -7.95491400 & 1.36300000 \\
$\mathrm{Mg}$ & 1.98752200 & -6.13391400 & 0.00000000 \\
$\mathrm{Cl}$ & 3.03852200 & -4.31391400 & 1.36300000 \\
$\mathrm{Mg}$ & 1.98752200 & -2.49291400 & 0.00000000 \\
$\mathrm{Cl}$ & 3.03852200 & -0.67291400 & 1.36300000 \\
$\mathrm{Mg}$ & 1.98752200 & 1.14808600 & 0.00000000 \\
$\mathrm{C}$ & \multicolumn{3}{c}{}
\end{tabular}

$\mathrm{Mg}_{64} \mathrm{Cl}_{\mathrm{Mg8}}$ (Frozen)

Input Keywords:

\#p hf/Gen geom=connectivity scfcyc=800 MaxDisk=40GB Input coordinates:

$\begin{array}{llll}\mathrm{Cl} & -17.98247800 & -7.95491400 & -1.36300000 \\ \mathrm{Cl} & -17.98247800 & -4.31391400 & -1.36300000 \\ \mathrm{Cl} & -17.98247800 & -0.67291400 & -1.36300000 \\ \mathrm{Cl} & -17.98247800 & 2.96808600 & -1.36300000 \\ \mathrm{Cl} & -17.98247800 & 6.60908600 & -1.36300000 \\ \mathrm{Cl} & -17.98247800 & 10.25008600 & -1.36300000 \\ \mathrm{Cl} & -17.98247800 & 13.89108600 & -1.36300000 \\ \mathrm{Cl} & -17.98247800 & 17.53208600 & -1.36300000 \\ \mathrm{Cl} & -15.88047800 & -11.59591400 & 1.36300000 \\ \mathrm{Mg} & -16.93147800 & -9.77491400 & 0.00000000 \\ \mathrm{Cl} & -15.88047800 & -7.95491400 & 1.36300000 \\ \mathrm{Cl} & -14.82947800 & -9.77491400 & -1.36300000 \\ \mathrm{Mg} & -16.93147800 & -6.13391400 & 0.00000000\end{array}$




\begin{tabular}{|c|c|c|}
\hline $\mathrm{Cl}$ & $-15.88047800 \quad-4.31391400$ & 1.36300000 \\
\hline $\mathrm{Cl}$ & $-14.82947800 \quad-6.13391400$ & -1.36300000 \\
\hline $\mathrm{Mg}$ & $-16.93147800 \quad-2.49291400$ & 0.00000000 \\
\hline $\mathrm{Cl}$ & $-15.88047800 \quad-0.67291400$ & 1.36300000 \\
\hline $\mathrm{Cl}$ & $-14.82947800 \quad-2.49291400$ & -1.36300000 \\
\hline $\mathrm{Mg}$ & $-16.93147800 \quad 1.14808600$ & 0.00000000 \\
\hline $\mathrm{Cl}$ & $-15.88047800 \quad 2.96808600$ & 1.36300000 \\
\hline $\mathrm{Cl}$ & -14.82947800 & -1.36300000 \\
\hline $\mathrm{Mg}$ & $-16.93147800 \quad 4.78908600$ & 0.00000000 \\
\hline $\mathrm{Cl}$ & $-15.88047800 \quad 6.60908600$ & 1.36300000 \\
\hline $\mathrm{Cl}$ & -14.82947800 & -1.36300000 \\
\hline $\mathrm{Mg}$ & $-16.93147800 \quad 8.43008600$ & 0.00000000 \\
\hline $\mathrm{Cl}$ & $-15.88047800 \quad 10.25008600$ & 1.36300000 \\
\hline $\mathrm{Cl}$ & $-14.82947800 \quad 8.43008600$ & -1.36300000 \\
\hline $\mathrm{Mg}$ & $-16.93147800 \quad 12.07108600$ & $\begin{array}{ll}0 & 0.00000000\end{array}$ \\
\hline $\mathrm{Cl}$ & $-15.88047800 \quad 13.89108600$ & 1.36300000 \\
\hline $\mathrm{Cl}$ & $-14.82947800 \quad 12.07108600$ & -1.36300000 \\
\hline $\mathrm{Mg}$ & $-16.93147800 \quad 15.71208600$ & $\begin{array}{ll}0 & 0.00000000\end{array}$ \\
\hline $\mathrm{Cl}$ & $-14.82947800 \quad 15.71208600$ & -1.36300000 \\
\hline $\mathrm{Cl}$ & $-12.72747800-13.41691400$ & 1.36300000 \\
\hline $\mathrm{Mg}$ & $-13.77847800-11.59591400$ & $0 \quad 0.00000000$ \\
\hline $\mathrm{Cl}$ & $-12.72747800 \quad-9.77491400$ & 1.36300000 \\
\hline $\mathrm{Cl}$ & $-11.67547800-11.59591400$ & -1.36300000 \\
\hline $\mathrm{Mg}$ & $-13.77847800 \quad-7.95491400$ & 0.00000000 \\
\hline $\mathrm{Cl}$ & $-12.72747800 \quad-6.13391400$ & 1.36300000 \\
\hline $\mathrm{Cl}$ & $1547800 \quad-7.95491400$ & 300000 \\
\hline $\mathrm{Mg}$ & $-13.77847800 \quad-4.31391400$ & 00000 \\
\hline $\mathrm{Cl}$ & $-12.72747800 \quad-2.49291400$ & 00000 \\
\hline $\mathrm{Cl}$ & $-11.67547800 \quad-4.31391400$ & -1.36300000 \\
\hline $\mathrm{Mg}$ & $-13.77847800-0.67291400$ & 0.00000000 \\
\hline $\mathrm{Cl}$ & $-12.72747800 \quad 1.14808600$ & 1.36300000 \\
\hline $\mathrm{Cl}$ & $-11.67547800-0.67291400$ & -1.36300000 \\
\hline $\mathrm{Mg}$ & $-13.77847800 \quad 2.96808600$ & 0.00000000 \\
\hline $\mathrm{Cl}$ & $-12.72747800 \quad 4.78908600$ & 1.36300000 \\
\hline $\mathrm{Cl}$ & $-11.67547800 \quad 2.96808600$ & 0000 \\
\hline $\mathrm{Mg}$ & $-13.77847800 \quad 6.60908600$ & 0.00000000 \\
\hline $\mathrm{Cl}$ & $-12.72747800 \quad 8.43008600$ & 1.36300000 \\
\hline $\mathrm{Cl}$ & -11.67547800 & -1.36300000 \\
\hline $\mathrm{Mg}$ & $-13.77847800 \quad 10.25008600$ & $\begin{array}{ll}0 & 0.00000000\end{array}$ \\
\hline $\mathrm{Cl}$ & $-12.72747800 \quad 12.07108600$ & 1.36300000 \\
\hline $\mathrm{Cl}$ & $-11.67547800 \quad 10.25008600$ & -1.36300000 \\
\hline $\mathrm{Mg}$ & $-13.77847800 \quad 13.89108600$ & $\begin{array}{ll}0 & 0.00000000\end{array}$ \\
\hline $\mathrm{Cl}$ & $-11.67547800 \quad 13.89108600$ & -1.36300000 \\
\hline $\mathrm{Cl}$ & $-9.57347800-15.23691400$ & 1.36300000 \\
\hline $\mathrm{Mg}$ & $-10.62447800-13.41691400$ & $\begin{array}{ll}0 & 0.00000000\end{array}$ \\
\hline $\mathrm{Cl}$ & $-9.57347800-11.59591400$ & 1.36300000 \\
\hline $\mathrm{Cl}$ & 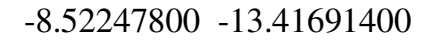 & -1.36300000 \\
\hline $\mathrm{Mg}$ & $-10.62447800 \quad-9.77491400$ & 0.00000000 \\
\hline $\mathrm{Cl}$ & $-9.57347800 \quad-7.95491400$ & 1.36300000 \\
\hline $\mathrm{Cl}$ & $-8.52247800 \quad-9.77491400$ & -1.36300000 \\
\hline $\mathrm{Mg}$ & $-10.62447800-6.13391400$ & 0.00000000 \\
\hline $\mathrm{Cl}$ & $-9.57347800 \quad-4.31391400$ & 1.36300000 \\
\hline $\mathrm{Cl}$ & $-8.52247800-6.13391400$ & -1.36300000 \\
\hline $\mathrm{Mg}$ & $-10.62447800-2.49291400$ & 0.00000000 \\
\hline $\mathrm{Cl}$ & $-9.57347800 \quad-0.67291400$ & 1.36300000 \\
\hline $\mathrm{Cl}$ & $-8.52247800-2.49291400$ & -1.36300000 \\
\hline $\mathrm{Mg}$ & $-10.62447800 \quad 1.14808600$ & 0.00000000 \\
\hline $\mathrm{Cl}$ & $-9.57347800 \quad 2.96808600$ & 1.36300000 \\
\hline $\mathrm{Cl}$ & -8.52247800 & -1.36300000 \\
\hline $\mathrm{Mg}$ & $-10.62447800 \quad 4.78908600$ & 0.00000000 \\
\hline $\mathrm{Cl}$ & 6.609086 & \\
\hline
\end{tabular}




\begin{tabular}{|c|c|}
\hline $\mathrm{Cl}$ & $\begin{array}{lll}-8.52247800 & 4.78908600 & -1.36300000\end{array}$ \\
\hline $\mathrm{Mg}$ & $\begin{array}{lll}-10.62447800 & 8.43008600 & 0.00000000\end{array}$ \\
\hline $\mathrm{Cl}$ & $\begin{array}{lll}-9.57347800 & 10.25008600 & 1.36300000\end{array}$ \\
\hline $\mathrm{Cl}$ & $\begin{array}{lll}-8.52247800 & 8.43008600 & -1.36300000\end{array}$ \\
\hline $\mathrm{Mg}$ & $\begin{array}{lll}-10.62447800 & 12.07108600 & 0.00000000\end{array}$ \\
\hline $\mathrm{Cl}$ & $\begin{array}{lll}-8.52247800 & 12.07108600 & -1.36300000\end{array}$ \\
\hline $\mathrm{Cl}$ & $\begin{array}{lll}-6.42047800 & -17.05791400 & 1.36300000\end{array}$ \\
\hline $\mathrm{Mg}$ & $\begin{array}{lll}-7.47147800 & -15.23691400 & 0.00000000\end{array}$ \\
\hline $\mathrm{Cl}$ & $\begin{array}{lll}-6.42047800 & -13.41691400 & 1.36300000\end{array}$ \\
\hline $\mathrm{Cl}$ & $\begin{array}{lll}-5.36947800 & -15.23691400 & -1.36300000\end{array}$ \\
\hline $\mathrm{Mg}$ & $\begin{array}{lll}-7.47147800 & -11.59591400 & 0.00000000\end{array}$ \\
\hline $\mathrm{Cl}$ & $\begin{array}{lll}-6.42047800 & -9.77491400 & 1.36300000\end{array}$ \\
\hline $\mathrm{Cl}$ & $\begin{array}{lll}-5.36947800 & -11.59591400 & -1.36300000\end{array}$ \\
\hline $\mathrm{Mg}$ & $\begin{array}{lll}-7.47147800 & -7.95491400 & 0.00000000\end{array}$ \\
\hline $\mathrm{Cl}$ & $\begin{array}{lll}-6.42047800 & -6.13391400 & 1.36300000\end{array}$ \\
\hline $\mathrm{Cl}$ & $\begin{array}{lll}-5.36947800 & -7.95491400 & -1.36300000\end{array}$ \\
\hline $\mathrm{Mg}$ & $\begin{array}{lll}-7.47147800 & -4.31391400 & 0.00000000\end{array}$ \\
\hline $\mathrm{Cl}$ & $\begin{array}{lll}-6.42047800 & -2.49291400 & 1.36300000\end{array}$ \\
\hline $\mathrm{Cl}$ & $\begin{array}{lll}-5.36947800 & -4.31391400 & -1.36300000\end{array}$ \\
\hline $\mathrm{Mg}$ & $\begin{array}{lll}-7.47147800 & -0.67291400 & 0.00000000\end{array}$ \\
\hline $\mathrm{Cl}$ & $\begin{array}{lll}-6.42047800 & 1.14808600 & 1.36300000\end{array}$ \\
\hline $\mathrm{Cl}$ & $\begin{array}{ccc}-5.36947800 & -0.67291400 & -1.36300000\end{array}$ \\
\hline $\mathrm{Mg}$ & $\begin{array}{lll}-7.47147800 & 2.96808600 & 0.00000000\end{array}$ \\
\hline $\mathrm{Cl}$ & $\begin{array}{lll}-6.42047800 & 4.78908600 & 1.36300000\end{array}$ \\
\hline $\mathrm{Cl}$ & $\begin{array}{lll}-5.36947800 & 2.96808600 & -1.36300000\end{array}$ \\
\hline $\mathrm{Mg}$ & $\begin{array}{lll}-7.47147800 & 6.60908600 & 0.00000000\end{array}$ \\
\hline $\mathrm{Cl}$ & $\begin{array}{lll}-6.42047800 & 8.43008600 & 1.36300000\end{array}$ \\
\hline $\mathrm{Cl}$ & $6.60908600-1.36$ \\
\hline $\mathrm{Mg}$ & $\begin{array}{lll}-7.47147800 & 10.25008600 & 0.00000000\end{array}$ \\
\hline $\mathrm{Cl}$ & $\begin{array}{lll}-5.36947800 & 10.25008600 & -1.36300000\end{array}$ \\
\hline $\mathrm{Cl}$ & $\begin{array}{lll}-3.26747800 & -18.87791400 & 1.36300000\end{array}$ \\
\hline $\mathrm{Mg}$ & $-4.31847800-17.05791400 \quad 0.00000000$ \\
\hline $\mathrm{Cl}$ & $\begin{array}{lll}-3.26747800 & -15.23691400 & 1.36300000\end{array}$ \\
\hline $\mathrm{Cl}$ & $-2.21647800-17.05791400-1.36$ \\
\hline $\mathrm{Mg}$ & $-4.31847800-13.41691400 \quad 0.00000000$ \\
\hline $\mathrm{Cl}$ & $\begin{array}{lll}-3.26747800 & -11.59591400 & 1.36300000\end{array}$ \\
\hline $\mathrm{Cl}$ & $\begin{array}{lll}-2.21647800 & -13.41691400 & -1.36300000\end{array}$ \\
\hline $\mathrm{Mg}$ & $\begin{array}{lll}-4.31847800 & -9.77491400 & 0.00000000\end{array}$ \\
\hline $\mathrm{Cl}$ & $-3.26747800 \quad-7.95491400 \quad 1.36300000$ \\
\hline $\mathrm{Cl}$ & $\begin{array}{lll}-2.21647800 & -9.77491400 & -1.36300000\end{array}$ \\
\hline $\mathrm{Mg}$ & $\begin{array}{lll}-4.31847800 & -6.13391400 & 0.00000000\end{array}$ \\
\hline $\mathrm{Cl}$ & $\begin{array}{lll}-3.26747800 & -4.31391400 & 1.36300000\end{array}$ \\
\hline $\mathrm{Cl}$ & $\begin{array}{lll}-2.21647800 & -6.13391400 & -1.363\end{array}$ \\
\hline $\mathrm{Mg}$ & $-4.31847800 \quad-2.49291400 \quad 0.00000000$ \\
\hline $\mathrm{Cl}$ & $\begin{array}{lll}-3.26747800 & -0.67291400 & 1.36300000\end{array}$ \\
\hline $\mathrm{Cl}$ & $\begin{array}{lll}-2.21647800 & -2.49291400 & -1.36300000\end{array}$ \\
\hline $\mathrm{Mg}$ & $-4.31847800 \quad 1.14808600 \quad 0.00000000$ \\
\hline $\mathrm{Cl}$ & $\begin{array}{lll}-3.26747800 & 2.96808600 & 1.36300000\end{array}$ \\
\hline $\mathrm{Cl}$ & $\begin{array}{lll}-2.21647800 & 1.14808600 & -1.36300000\end{array}$ \\
\hline $\mathrm{Mg}$ & $-4.31847800 \quad 4.78908600 \quad 0.00000000$ \\
\hline $\mathrm{Cl}$ & $\begin{array}{lll}-3.26747800 & 6.60908600 & 1.36300000\end{array}$ \\
\hline $\mathrm{Cl}$ & $4.78908600-1.36300000$ \\
\hline $\mathrm{Mg}$ & $-4.31847800 \quad 8.43008600 \quad 0.00000000$ \\
\hline $\mathrm{Cl}$ & $\begin{array}{lll}-2.21647800 & 8.43008600 & -1.36300000\end{array}$ \\
\hline $\mathrm{Cl}$ & $\begin{array}{lll}-0.11447800 & -20.69891400 & 1.36300000\end{array}$ \\
\hline $\mathrm{Mg}$ & $\begin{array}{lll}-1.16547800 & -18.87791400 & 0.00000000\end{array}$ \\
\hline $\mathrm{Cl}$ & $\begin{array}{lll}-0.11447800 & -17.05791400 & 1.36300000\end{array}$ \\
\hline $\mathrm{Cl}$ & $0.93652200-18.87791400 \quad-1.36300000$ \\
\hline $\mathrm{Mg}$ & $\begin{array}{lll}-1.16547800 & -15.23691400 & 0.00000000\end{array}$ \\
\hline $\mathrm{Cl}$ & $\begin{array}{lll}-0.11447800 & -13.41691400 & 1.36300000\end{array}$ \\
\hline $\mathrm{Cl}$ & -15.25691 \\
\hline
\end{tabular}




\begin{tabular}{|c|c|c|}
\hline $\mathrm{Mg}$ & $-1.16547800-11.59591400$ & 0.00000000 \\
\hline $\mathrm{Cl}$ & $-0.11447800 \quad-9.77491400$ & 1.36300000 \\
\hline $\mathrm{Cl}$ & $0.93652200-11.59591400$ & -1.36300000 \\
\hline $\mathrm{Mg}$ & $-1.16547800-7.95491400$ & 0.00000000 \\
\hline $\mathrm{Cl}$ & $-0.11447800-6.13391400$ & 1.36300000 \\
\hline $\mathrm{Cl}$ & $0.93652200-7.95491400$ & -1.36300000 \\
\hline $\mathrm{Mg}$ & $-1.16547800-4.31391400$ & 0.00000000 \\
\hline $\mathrm{Cl}$ & $-0.11447800 \quad-2.49291400$ & 1.36300000 \\
\hline $\mathrm{Cl}$ & $0.93652200-4.31391400$ & -1.36300000 \\
\hline $\mathrm{Mg}$ & $-1.16547800 \quad-0.67291400$ & 0.00000000 \\
\hline $\mathrm{Cl}$ & $-0.11447800 \quad 1.14808600$ & 1.36300000 \\
\hline $\mathrm{Cl}$ & $0.93652200-0.67291400$ & -1.36300000 \\
\hline $\mathrm{Mg}$ & $-1.16547800 \quad 2.96808600$ & 0.00000000 \\
\hline $\mathrm{Cl}$ & $-0.11447800 \quad 4.78908600$ & 1.36300000 \\
\hline $\mathrm{Cl}$ & 2.96808600 & -1.36300000 \\
\hline $\mathrm{Mg}$ & $-1.16547800 \quad 6.60908600$ & 0.00000000 \\
\hline $\mathrm{Cl}$ & $0.93652200 \quad 6.60908600$ & -1.36300000 \\
\hline $\mathrm{Cl}$ & $3.03852200-22.51891400$ & 1.36300000 \\
\hline $\mathrm{Mg}$ & $1.98752200-20.69891400$ & 0.00000000 \\
\hline $\mathrm{Cl}$ & $3.03852200-18.87791400$ & 1.36300000 \\
\hline $\mathrm{Cl}$ & $4.09052200-20.69891400$ & -1.36300000 \\
\hline $\mathrm{Mg}$ & $1.98752200-17.05791400$ & 0.00000000 \\
\hline $\mathrm{Cl}$ & $3.03852200-15.23691400$ & 1.36300000 \\
\hline $\mathrm{Cl}$ & $4.09052200-17.05791400$ & -1.36300000 \\
\hline $\mathrm{Mg}$ & $1.98752200-13.41691400$ & 0.00000000 \\
\hline $\mathrm{Cl}$ & $3.03852200-11.59591400$ & 1.36300000 \\
\hline $\mathrm{Cl}$ & $4.09052200-13.41691400$ & -1.36300000 \\
\hline $\mathrm{Mg}$ & $1.98752200 \quad-9.77491400$ & 0.00000000 \\
\hline $\mathrm{Cl}$ & $3.03852200 \quad-7.95491400$ & 1.36300000 \\
\hline $\mathrm{Cl}$ & $4.09052200 \quad-9.77491400$ & -1.36300000 \\
\hline $\mathrm{Mg}$ & $1.98752200-6.13391400$ & 0.00000000 \\
\hline $\mathrm{Cl}$ & $3.03852200 \quad-4.31391400$ & 1.36300000 \\
\hline $\mathrm{Cl}$ & $4.09052200 \quad-6.13391400$ & -1.36300000 \\
\hline $\mathrm{Mg}$ & $1.98752200 \quad-2.49291400$ & 0.00000000 \\
\hline $\mathrm{Cl}$ & $3.03852200-0.67291400$ & 1.36300000 \\
\hline $\mathrm{Cl}$ & $4.09052200 \quad-2.49291400$ & -1.36300000 \\
\hline $\mathrm{Mg}$ & $1.98752200 \quad 1.14808600$ & 0.00000000 \\
\hline $\mathrm{Cl}$ & $3.03852200 \quad 2.96808600$ & 1.36300000 \\
\hline $\mathrm{Cl}$ & $4.09052200 \quad 1.14808600$ & -1.36300000 \\
\hline $\mathrm{Mg}$ & $1.98752200 \quad 4.78908600$ & 0.00000000 \\
\hline $\mathrm{Cl}$ & $4.09052200 \quad 4.78908600$ & -1.36300000 \\
\hline $\mathrm{Cl}$ & $6.19252200 \quad-24.33991400$ & 1.36300000 \\
\hline $\mathrm{Mg}$ & $5.14152200-22.51891400$ & 0.00000000 \\
\hline $\mathrm{Cl}$ & $6.19252200-20.69891400$ & 1.36300000 \\
\hline $\mathrm{Mg}$ & $5.14152200-18.87791400$ & 0.00000000 \\
\hline $\mathrm{Cl}$ & $6.19252200-17.05791400$ & 1.36300000 \\
\hline $\mathrm{Mg}$ & $5.14152200-15.23691400$ & 0.00000000 \\
\hline $\mathrm{Cl}$ & $6.19252200-13.41691400$ & 1.36300000 \\
\hline $\mathrm{Mg}$ & $5.14152200-11.59591400$ & 0.00000000 \\
\hline $\mathrm{Cl}$ & $6.19252200 \quad-9.77491400$ & 1.36300000 \\
\hline $\mathrm{Mg}$ & $5.14152200 \quad-7.95491400$ & 0.00000000 \\
\hline $\mathrm{Cl}$ & $6.19252200-6.13391400$ & 1.36300000 \\
\hline $\mathrm{Mg}$ & $5.14152200-4.31391400$ & 0.00000000 \\
\hline $\mathrm{Cl}$ & $6.19252200 \quad-2.49291400$ & 1.36300000 \\
\hline $\mathrm{Mg}$ & $5.14152200 \quad-0.67291400$ & 0.00000000 \\
\hline $\mathrm{Cl}$ & $6.19252200 \quad 1.14808600$ & 1.36300000 \\
\hline $\mathrm{Mg}$ & $5.14152200 \quad 2.96808600$ & 0.00000000 \\
\hline $\mathrm{Mg}$ & $5.14152200 \quad 2.96808600$ & 0.00000000 \\
\hline
\end{tabular}

$\mathrm{Mg}_{81} \mathrm{Cl}_{162}$ (Frozen) 
Input Keywords:

\#p hf/Gen geom=connectivity scfcyc=800 MaxDisk=40GB Input coordinates:

Cl $\quad \quad-17.98247800 \quad-7.95491400 \quad-1.36300000$

$\mathrm{Cl} \quad-17.98247800 \quad-4.31391400-1.36300000$

$\mathrm{Cl} \quad-17.98247800 \quad-0.67291400 \quad-1.36300000$

$\begin{array}{lllll}\mathrm{Cl} & -17.98247800 & 2.96808600 & -1.36300000\end{array}$

$\begin{array}{lllll}\mathrm{Cl} & -17.98247800 & 6.60908600 & -1.36300000\end{array}$

$\mathrm{Cl} \quad \begin{array}{llll}\mathrm{C} & -17.98247800 & 10.25008600 & -1.36300000\end{array}$

$\mathrm{Cl} \quad \begin{array}{llll}\mathrm{Cl} & -17.98247800 & 13.89108600 & -1.36300000\end{array}$

$\begin{array}{llll}\mathrm{Cl} & -17.98247800 & 17.53208600 & -1.36300000\end{array}$

Cl $\quad \begin{array}{llll}\text { Cl } & -17.98247800 & 21.17308600 & -1.36300000\end{array}$

$\begin{array}{llll}\mathrm{Cl} & -15.88047800 & -11.59591400 & 1.36300000\end{array}$

$\begin{array}{llll}\mathrm{Mg} & -16.93147800 & -9.77491400 & 0.00000000\end{array}$

$\begin{array}{llll}\mathrm{Cl} & -15.88047800 & -7.95491400 & 1.36300000\end{array}$

$\mathrm{Cl} \quad-14.82947800 \quad-9.77491400 \quad-1.36300000$

$\begin{array}{llll}\mathrm{Mg} & -16.93147800 & -6.13391400 & 0.00000000\end{array}$

$\begin{array}{llll}\mathrm{Cl} & -15.88047800 & -4.31391400 & 1.36300000\end{array}$

$\mathrm{Cl} \quad-14.82947800 \quad-6.13391400 \quad-1.36300000$

$\begin{array}{llll}\mathrm{Mg} & -16.93147800 & -2.49291400 & 0.00000000\end{array}$

$\mathrm{Cl} \quad-15.88047800 \quad-0.67291400 \quad 1.36300000$

$\mathrm{Cl} \quad-14.82947800 \quad-2.49291400 \quad-1.36300000$

$\begin{array}{llll}\mathrm{Mg} & -16.93147800 & 1.14808600 & 0.00000000\end{array}$

$\begin{array}{llll}\mathrm{Cl} & -15.88047800 & 2.96808600 & 1.36300000\end{array}$

$\begin{array}{lllll}\mathrm{Cl} & & -14.82947800 & 1.14808600 & -1.36300000\end{array}$

$\begin{array}{lllll}\mathrm{Mg} & -16.93147800 & 4.78908600 & 0.00000000\end{array}$

$\begin{array}{lllll}\mathrm{Cl} & -15.88047800 & 6.60908600 & 1.36300000\end{array}$

$\mathrm{Cl} \quad \quad-14.82947800 \quad 4.78908600 \quad-1.36300000$

$\begin{array}{llll}\mathrm{Mg} & -16.93147800 & 8.43008600 & 0.00000000\end{array}$

$\begin{array}{lllll}\mathrm{Cl} & & -15.88047800 & 10.25008600 & 1.36300000\end{array}$

$\begin{array}{lllll}\mathrm{Cl} & -14.82947800 & 8.43008600 & -1.36300000\end{array}$

$\begin{array}{lllll}\mathrm{Mg} & & -16.93147800 & 12.07108600 & 0.00000000\end{array}$

$\begin{array}{lllll}\mathrm{Cl} & & -15.88047800 & 13.89108600 & 1.36300000\end{array}$

$\begin{array}{llll}\mathrm{Cl} & -14.82947800 & 12.07108600 & -1.36300000\end{array}$

$\begin{array}{llll}\mathrm{Mg} & -16.93147800 & 15.71208600 & 0.00000000\end{array}$

$\begin{array}{lllll}\mathrm{Cl} & & -15.88047800 & 17.53208600 & 1.36300000\end{array}$

$\begin{array}{lllll}\mathrm{Cl} & & -14.82947800 & 15.71208600 & -1.36300000\end{array}$

$\begin{array}{lllll}\mathrm{Mg} & & -16.93147800 & 19.35308600 & 0.00000000\end{array}$

$\begin{array}{lllll}\mathrm{Cl} & & -14.82947800 & 19.35308600 & -1.36300000\end{array}$

$\begin{array}{lllll}\mathrm{Cl} & & -12.72747800 & -13.41691400 & 1.36300000\end{array}$

$\begin{array}{llll}\mathrm{Mg} & -13.77847800 & -11.59591400 & 0.00000000\end{array}$

$\begin{array}{lllll}\mathrm{Cl} & -12.72747800 & -9.77491400 & 1.36300000\end{array}$

$\mathrm{Cl} \quad-11.67547800 \quad-11.59591400 \quad-1.36300000$

$\begin{array}{llll}\mathrm{Mg} & -13.77847800 & -7.95491400 & 0.00000000\end{array}$

$\mathrm{Cl} \quad-12.72747800 \quad-6.13391400 \quad 1.36300000$

$\mathrm{Cl} \quad-11.67547800 \quad-7.95491400 \quad-1.36300000$

$\begin{array}{llll}\mathrm{Mg} & -13.77847800 & -4.31391400 & 0.00000000\end{array}$

$\mathrm{Cl} \quad-12.72747800 \quad-2.49291400 \quad 1.36300000$

$\mathrm{Cl} \quad-11.67547800 \quad-4.31391400 \quad-1.36300000$

$\begin{array}{llll}\mathrm{Mg} & -13.77847800 & -0.67291400 & 0.00000000\end{array}$

$\begin{array}{llll}\mathrm{Cl} & -12.72747800 & 1.14808600 & 1.36300000\end{array}$

$\mathrm{Cl} \quad-11.67547800 \quad-0.67291400 \quad-1.36300000$

$\begin{array}{llll}\mathrm{Mg} & -13.77847800 & 2.96808600 & 0.00000000\end{array}$

$\begin{array}{llll}\mathrm{Cl} & -12.72747800 & 4.78908600 & 1.36300000\end{array}$

$\mathrm{Cl} \quad \begin{array}{llll}\mathrm{Cl} & -11.67547800 & 2.96808600 & -1.36300000\end{array}$

$\begin{array}{lllll}\mathrm{Mg} & -13.77847800 & 6.60908600 & 0.00000000\end{array}$

$\begin{array}{lllll}\mathrm{Cl} & -12.72747800 & 8.43008600 & 1.36300000\end{array}$

$\begin{array}{lllll}\mathrm{Cl} & -11.67547800 & 6.60908600 & -1.36300000\end{array}$

$\begin{array}{lllll}\mathrm{Mg} & -13.77847800 & 10.25008600 & 0.00000000\end{array}$

$\begin{array}{llll}\mathrm{Cl} & -12.72747800 & 12.07108600 & 1.36300000\end{array}$ 


\begin{tabular}{|c|c|}
\hline $\mathrm{Cl}$ & $\begin{array}{lll}-11.67547800 & 10.25008600 & -1.36300000\end{array}$ \\
\hline $\mathrm{Mg}$ & $\begin{array}{lll}-13.77847800 & 13.89108600 & 0.00000000\end{array}$ \\
\hline $\mathrm{Cl}$ & $-12.72747800 \quad 15.71208600$ \\
\hline $\mathrm{Cl}$ & $\begin{array}{lll}-11.67547800 & 13.89108600 & -1.36300000\end{array}$ \\
\hline $\mathrm{Mg}$ & $\begin{array}{lll}-13.77847800 & 17.53208600 & 0.00000000\end{array}$ \\
\hline $\mathrm{Cl}$ & $\begin{array}{lll}-11.67547800 & 17.53208600 & -1.36300000\end{array}$ \\
\hline $\mathrm{Cl}$ & $\begin{array}{lll}-9.57347800 & -15.23691400 & 1.36300000\end{array}$ \\
\hline $\mathrm{Mg}$ & $\begin{array}{lll}-10.62447800 & -13.41691400 & 0.00000000\end{array}$ \\
\hline $\mathrm{Cl}$ & $\begin{array}{lll}-9.57347800 & -11.59591400 & 1.36300000\end{array}$ \\
\hline $\mathrm{Cl}$ & $\begin{array}{lll}-8.52247800 & -13.41691400 & -1.36300000\end{array}$ \\
\hline $\mathrm{Mg}$ & $\begin{array}{lll}-10.62447800 & -9.77491400 & 0.00000000\end{array}$ \\
\hline $\mathrm{Cl}$ & $\begin{array}{lll}-9.57347800 & -7.95491400 & 1.36300000\end{array}$ \\
\hline $\mathrm{Cl}$ & $\begin{array}{lll}-8.52247800 & -9.77491400 & -1.36300000\end{array}$ \\
\hline $\mathrm{Mg}$ & $-10.62447800-6.13391400 \quad 0.00000000$ \\
\hline $\mathrm{Cl}$ & $\begin{array}{lll}-9.57347800 & -4.31391400 & 1.36300000\end{array}$ \\
\hline $\mathrm{Cl}$ & $\begin{array}{lll}-8.52247800 & -6.13391400 & -1.36300000\end{array}$ \\
\hline $\mathrm{Mg}$ & $\begin{array}{lll}-10.62447800 & -2.49291400 & 0.00000000\end{array}$ \\
\hline $\mathrm{Cl}$ & $\begin{array}{lll}-9.57347800 & -0.67291400 & 1.36300000\end{array}$ \\
\hline $\mathrm{Cl}$ & $\begin{array}{lll}-8.52247800 & -2.49291400 & -1.36300000\end{array}$ \\
\hline $\mathrm{Mg}$ & $\begin{array}{lll}-10.62447800 & 1.14808600 & 0.00000000\end{array}$ \\
\hline $\mathrm{Cl}$ & $\begin{array}{lll}-9.57347800 & 2.96808600 & 1.36300000\end{array}$ \\
\hline $\mathrm{Cl}$ & $1.14808600-1.36300000$ \\
\hline $\mathrm{Mg}$ & $\begin{array}{lll}-10.62447800 & 4.78908600 & 0.00000000\end{array}$ \\
\hline $\mathrm{Cl}$ & $\begin{array}{lll}-9.57347800 & 6.60908600 & 1.36300000\end{array}$ \\
\hline $\mathrm{Cl}$ & $\begin{array}{lll}-8.52247800 & 4.78908600 & -1.36300000\end{array}$ \\
\hline $\mathrm{Mg}$ & $\begin{array}{lll}-10.62447800 & 8.43008600 & 0.00000000\end{array}$ \\
\hline $\mathrm{Cl}$ & $\begin{array}{lll}-9.57347800 & 10.25008600 & 1.36300000\end{array}$ \\
\hline $\mathrm{Cl}$ & $8.43008600-1.363$ \\
\hline $\mathrm{Mg}$ & $\begin{array}{lll}-10.62447800 & 12.07108600 & 0.00000000\end{array}$ \\
\hline $\mathrm{Cl}$ & $\begin{array}{lll}-9.57347800 & 13.89108600 & 1.36300000\end{array}$ \\
\hline $\mathrm{Cl}$ & $\begin{array}{llll}-8.52247800 & 12.07108600 & -1.36300000\end{array}$ \\
\hline $\mathrm{Mg}$ & $\begin{array}{lll}-10.62447800 & 15.71208600 & 0.00000000\end{array}$ \\
\hline $\mathrm{Cl}$ & $\begin{array}{lll}-8.52247800 & 15.71208600 & -1.36300000\end{array}$ \\
\hline $\mathrm{Cl}$ & $\begin{array}{lll}-6.42047800 & -17.05791400 & 1.36300000\end{array}$ \\
\hline $\mathrm{Mg}$ & $-7.47147800-15.23691400 \quad 0.0$ \\
\hline $\mathrm{Cl}$ & $\begin{array}{lll}-6.42047800 & -13.41691400 & 1.36300000\end{array}$ \\
\hline $\mathrm{Cl}$ & $\begin{array}{lll}-5.36947800 & -15.23691400 & -1.36300000\end{array}$ \\
\hline $\mathrm{Mg}$ & $\begin{array}{lll}-7.47147800 & -11.59591400 & 0.00000000\end{array}$ \\
\hline $\mathrm{Cl}$ & $\begin{array}{lll}-6.42047800 & -9.77491400 & 1.36300000\end{array}$ \\
\hline $\mathrm{Cl}$ & $\begin{array}{lll}-5.36947800 & -11.59591400 & -1.36300000\end{array}$ \\
\hline $\mathrm{Mg}$ & $\begin{array}{lll}-7.47147800 & -7.95491400 & 0.00000000\end{array}$ \\
\hline $\mathrm{Cl}$ & $\begin{array}{lll}-6.42047800 & -6.13391400 & 1.36300000\end{array}$ \\
\hline $\mathrm{Cl}$ & $\begin{array}{lll}-5.36947800 & -7.95491400 & -1.36\end{array}$ \\
\hline $\mathrm{Mg}$ & $\begin{array}{lll}-7.47147800 & -4.31391400 & 0.00000000\end{array}$ \\
\hline $\mathrm{Cl}$ & $\begin{array}{lll}-6.42047800 & -2.49291400 & 1.36300000\end{array}$ \\
\hline $\mathrm{Cl}$ & $-4.31391400-1.36300000$ \\
\hline $\mathrm{Mg}$ & $\begin{array}{lll}-7.47147800 & -0.67291400 & 0.00000000\end{array}$ \\
\hline $\mathrm{Cl}$ & $\begin{array}{lll}-6.42047800 & 1.14808600 & 1.36300000\end{array}$ \\
\hline $\mathrm{Cl}$ & $\begin{array}{lll}-5.36947800 & -0.67291400 & -1.36300000\end{array}$ \\
\hline $\mathrm{Mg}$ & $\begin{array}{lll}-7.47147800 & 2.96808600 & 0.00000000\end{array}$ \\
\hline $\mathrm{Cl}$ & $\begin{array}{lll}-6.42047800 & 4.78908600 & 1.36300000\end{array}$ \\
\hline $\mathrm{Cl}$ & $\begin{array}{lll}-5.36947800 & 2.96808600 & -1.36300000\end{array}$ \\
\hline $\mathrm{Mg}$ & $\begin{array}{lll}-7.47147800 & 6.60908600 & 0.00000000\end{array}$ \\
\hline $\mathrm{Cl}$ & $\begin{array}{lll}-6.42047800 & 8.43008600 & 1.36300000\end{array}$ \\
\hline $\mathrm{Cl}$ & $\begin{array}{lll}-5.36947800 & 6.60908600 & -1.36300000\end{array}$ \\
\hline $\mathrm{Mg}$ & $\begin{array}{lll}-7.47147800 & 10.25008600 & 0.00000000\end{array}$ \\
\hline $\mathrm{Cl}$ & $\begin{array}{lll}-6.42047800 & 12.07108600 & 1.36300000\end{array}$ \\
\hline $\mathrm{Cl}$ & $\begin{array}{lll}-5.36947800 & 10.25008600 & -1.36300000\end{array}$ \\
\hline $\mathrm{Mg}$ & $\begin{array}{llll}-7.47147800 & 13.89108600 & 0.00000000\end{array}$ \\
\hline $\mathrm{Cl}$ & $\begin{array}{lll}-5.36947800 & 13.89108600 & -1.36300000\end{array}$ \\
\hline $\mathrm{Cl}$ & $-3.26 / 4 / 800-18.8 / / 91400$ \\
\hline
\end{tabular}




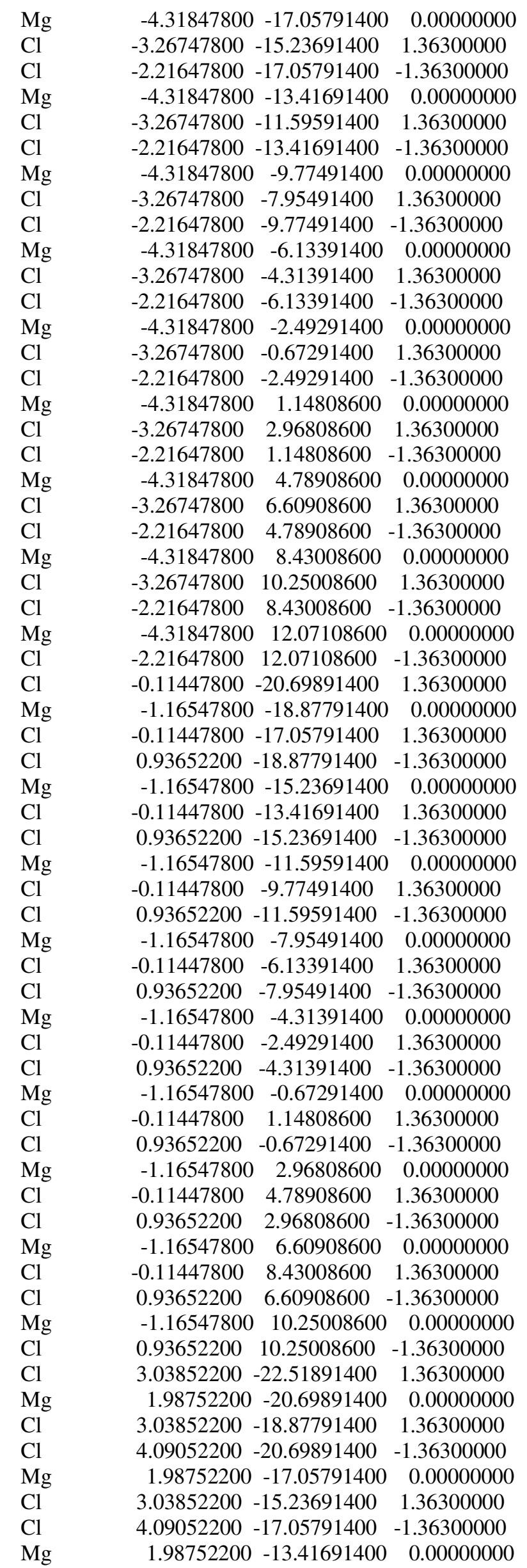




\begin{tabular}{|c|c|c|}
\hline $\mathrm{Cl}$ & $3.03852200-11.59591400$ & 1.36300000 \\
\hline $\mathrm{Cl}$ & $\begin{array}{lll}4.09052200 & -13.41691400\end{array}$ & -1.36300000 \\
\hline $\mathrm{Mg}$ & $1.98752200 \quad-9.77491400$ & 0.00000000 \\
\hline $\mathrm{Cl}$ & $3.03852200 \quad-7.95491400$ & 1.36300000 \\
\hline $\mathrm{Cl}$ & $4.09052200 \quad-9.77491400$ & -1.36300000 \\
\hline $\mathrm{Mg}$ & $1.98752200 \quad-6.13391400$ & 0.00000000 \\
\hline $\mathrm{Cl}$ & $3.03852200-4.31391400$ & 1.36300000 \\
\hline $\mathrm{Cl}$ & $4.09052200-6.13391400$ & -1.36300000 \\
\hline $\mathrm{Mg}$ & $1.98752200 \quad-2.49291400$ & 0.00000000 \\
\hline $\mathrm{Cl}$ & $3.03852200 \quad-0.67291400$ & 1.36300000 \\
\hline $\mathrm{Cl}$ & $4.09052200 \quad-2.49291400$ & -1.36300000 \\
\hline $\mathrm{Mg}$ & $1.98752200 \quad 1.14808600$ & 0.00000000 \\
\hline $\mathrm{Cl}$ & $3.03852200 \quad 2.96808600$ & 1.36300000 \\
\hline $\mathrm{Cl}$ & 4.09052200 & -1.36300000 \\
\hline $\mathrm{Mg}$ & $1.98752200 \quad 4.78908600$ & 0.00000000 \\
\hline $\mathrm{Cl}$ & $3.03852200 \quad 6.60908600$ & 1.36300000 \\
\hline $\mathrm{Cl}$ & 4.09052200 & -1.36300000 \\
\hline $\mathrm{Mg}$ & $1.98752200 \quad 8.43008600$ & 0.00000000 \\
\hline $\mathrm{Cl}$ & 4.090522008 .43008600 & -1.36300000 \\
\hline $\mathrm{Cl}$ & $6.19252200-24.33991400$ & 1.36300000 \\
\hline $\mathrm{Mg}$ & $5.14152200-22.51891400$ & 0.00000000 \\
\hline $\mathrm{Cl}$ & $6.19252200-20.69891400$ & 1.36300000 \\
\hline $\mathrm{Cl}$ & $7.24352200-22.51891400$ & -1.36300000 \\
\hline $\mathrm{Mg}$ & $5.14152200-18.87791400$ & 0.00000000 \\
\hline $\mathrm{Cl}$ & $6.19252200-17.05791400$ & 1.36300000 \\
\hline $\mathrm{Cl}$ & $7.24352200-18.87791400$ & -1.36300000 \\
\hline $\mathrm{Mg}$ & $5.14152200-15.23691400$ & 0.00000000 \\
\hline $\mathrm{Cl}$ & $6.19252200-13.41691400$ & 1.36300000 \\
\hline $\mathrm{Cl}$ & $7.24352200-15.23691400$ & -1.36300000 \\
\hline $\mathrm{Mg}$ & $5.14152200-11.59591400$ & 0.00000000 \\
\hline $\mathrm{Cl}$ & $6.19252200 \quad-9.77491400$ & 1.36300000 \\
\hline $\mathrm{Cl}$ & $\begin{array}{lll}7.24352200 & -11.59591400\end{array}$ & -1.36300000 \\
\hline $\mathrm{Mg}$ & $5.14152200 \quad-7.95491400$ & 0.00000000 \\
\hline $\mathrm{Cl}$ & $6.19252200-6.13391400$ & 1.36300000 \\
\hline $\mathrm{Cl}$ & $7.24352200 \quad-7.95491400$ & -1.36300000 \\
\hline $\mathrm{Mg}$ & $5.14152200 \quad-4.31391400$ & 0.00000000 \\
\hline $\mathrm{Cl}$ & $6.19252200-2.49291400$ & 1.36300000 \\
\hline $\mathrm{Cl}$ & $7.24352200 \quad-4.31391400$ & -1.36300000 \\
\hline $\mathrm{Mg}$ & $5.14152200-0.67291400$ & 0.00000000 \\
\hline $\mathrm{Cl}$ & $6.19252200 \quad 1.14808600$ & 1.36300000 \\
\hline $\mathrm{Cl}$ & $7.24352200 \quad-0.67291400$ & -1.36300000 \\
\hline $\mathrm{Mg}$ & $5.14152200 \quad 2.96808600$ & 0.00000000 \\
\hline $\mathrm{Cl}$ & $6.19252200 \quad 4.78908600$ & 1.36300000 \\
\hline $\mathrm{Cl}$ & $7.24352200 \quad 2.96808600$ & -1.36300000 \\
\hline $\mathrm{Mg}$ & $5.14152200 \quad 6.60908600$ & 0.00000000 \\
\hline $\mathrm{Cl}$ & $7.24352200 \quad 6.60908600$ & -1.36300000 \\
\hline $\mathrm{Cl}$ & $9.34552200-26.15991400$ & 1.36300000 \\
\hline $\mathrm{Mg}$ & $8.29452200-24.33991400$ & 0.00000000 \\
\hline $\mathrm{Cl}$ & $9.34552200-22.51891400$ & 1.36300000 \\
\hline $\mathrm{Mg}$ & $8.29452200-20.69891400$ & 0.00000000 \\
\hline $\mathrm{Cl}$ & $\begin{array}{lll}9.34552200 & -18.87791400\end{array}$ & 1.36300000 \\
\hline $\mathrm{Mg}$ & $8.29452200-17.05791400$ & 0.00000000 \\
\hline $\mathrm{Cl}$ & $9.34552200-15.23691400$ & 1.36300000 \\
\hline $\mathrm{Mg}$ & $8.29452200-13.41691400$ & 0.00000000 \\
\hline $\mathrm{Cl}$ & $\begin{array}{lll}9.34552200 & -11.59591400\end{array}$ & 1.36300000 \\
\hline $\mathrm{Mg}$ & $8.29452200 \quad-9.77491400$ & 0.00000000 \\
\hline $\mathrm{Cl}$ & $9.34552200 \quad-7.95491400$ & 1.36300000 \\
\hline $\mathrm{Mg}$ & $8.29452200 \quad-6.13391400$ & 0.00000000 \\
\hline $\mathrm{Cl}$ & $9.34552200 \quad-4.31391400$ & 1.36300000 \\
\hline $\mathrm{Mg}$ & $8.29452200 \quad-2.49291400$ & 0.00000000 \\
\hline $\mathrm{Cl}$ & $.34552200 \quad-0.67291400$ & 1.36300000 \\
\hline
\end{tabular}




$\begin{array}{lccc}\mathrm{Mg} & 8.29452200 & 1.14808600 & 0.00000000 \\ \mathrm{Cl} & 9.34552200 & 2.96808600 & 1.36300000 \\ \mathrm{Mg} & 8.29452200 & 4.78908600 & 0.00000000\end{array}$

\section{$\mathrm{Mg}_{100} \mathrm{Cl}_{200}$ (Frozen)}

Input Keywords:

$\#$ p hf/gen geom=connectivity scfcyc=800 MaxDisk=40GB Input coordinates:

\begin{tabular}{|c|c|c|c|}
\hline $\mathrm{Cl}$ & -17.98247800 & -7.95491400 & -1.36300000 \\
\hline $\mathrm{Cl}$ & -17.98247800 & -4.31391400 & -1.36300000 \\
\hline $\mathrm{Cl}$ & -17.98247800 & -0.67291400 & -1.36300000 \\
\hline $\mathrm{Cl}$ & -17.98247800 & 2.96808600 & -1.36300000 \\
\hline $\mathrm{Cl}$ & -17.98247800 & 6.60908600 & -1.36300000 \\
\hline $\mathrm{Cl}$ & -17.98247800 & 10.25008600 & -1.36300000 \\
\hline $\mathrm{Cl}$ & -17.98247800 & 13.89108600 & -1.36300000 \\
\hline $\mathrm{Cl}$ & -17.98247800 & 17.53208600 & -1.36300000 \\
\hline $\mathrm{Cl}$ & -17.98247800 & 21.17308600 & -1.36300000 \\
\hline $\mathrm{Cl}$ & -17.98247800 & 24.81408600 & -1.36300000 \\
\hline $\mathrm{Cl}$ & -15.88047800 & -11.59591400 & 1.36300000 \\
\hline $\mathrm{Mg}$ & -16.93147800 & -9.77491400 & 0.00000000 \\
\hline $\mathrm{Cl}$ & -15.88047800 & -7.95491400 & 1.36300000 \\
\hline $\mathrm{Cl}$ & -14.82947800 & -9.77491400 & -1.36300000 \\
\hline $\mathrm{Mg}$ & -16.93147800 & -6.13391400 & 0.00000000 \\
\hline $\mathrm{Cl}$ & -15.88047800 & -4.31391400 & 1.36300000 \\
\hline $\mathrm{Cl}$ & -14.82947800 & -6.13391400 & -1.36300000 \\
\hline $\mathrm{Mg}$ & -16.93147800 & -2.49291400 & 0.00000000 \\
\hline $\mathrm{Cl}$ & -15.88047800 & -0.67291400 & 1.36300000 \\
\hline $\mathrm{Cl}$ & -14.82947800 & -2.49291400 & -1.36300000 \\
\hline $\mathrm{Mg}$ & -16.93147800 & 1.14808600 & 0.00000000 \\
\hline $\mathrm{Cl}$ & -15.88047800 & 2.96808600 & 1.36300000 \\
\hline $\mathrm{Cl}$ & -14.82947800 & 1.14808600 & -1.36300000 \\
\hline $\mathrm{Mg}$ & -16.93147800 & 4.78908600 & 0.00000000 \\
\hline $\mathrm{Cl}$ & -15.88047800 & 6.60908600 & 1.36300000 \\
\hline $\mathrm{Cl}$ & -14.82947800 & 4.78908600 & -1.36300000 \\
\hline $\mathrm{Mg}$ & -16.93147800 & 8.43008600 & 0.00000000 \\
\hline $\mathrm{Cl}$ & -15.88047800 & 10.25008600 & 1.36300000 \\
\hline $\mathrm{Cl}$ & -14.82947800 & 8.43008600 & -1.36300000 \\
\hline $\mathrm{Mg}$ & -16.93147800 & 12.07108600 & 0.00000000 \\
\hline $\mathrm{Cl}$ & -15.88047800 & 13.89108600 & 1.36300000 \\
\hline $\mathrm{Cl}$ & -14.82947800 & 12.07108600 & -1.36300000 \\
\hline $\mathrm{Mg}$ & -16.93147800 & 15.71208600 & 0.00000000 \\
\hline $\mathrm{Cl}$ & -15.88047800 & 17.53208600 & 1.36300000 \\
\hline $\mathrm{Cl}$ & -14.82947800 & 15.71208600 & -1.36300000 \\
\hline $\mathrm{Mg}$ & -16.93147800 & 19.35308600 & 0.00000000 \\
\hline $\mathrm{Cl}$ & -15.88047800 & 21.17308600 & 1.36300000 \\
\hline $\mathrm{Cl}$ & -14.82947800 & 19.35308600 & -1.36300000 \\
\hline $\mathrm{Mg}$ & -16.93147800 & 22.99408600 & 0.00000000 \\
\hline $\mathrm{Cl}$ & -14.82947800 & 22.99408600 & -1.36300000 \\
\hline $\mathrm{Cl}$ & -12.72747800 & -13.41691400 & 1.36300000 \\
\hline $\mathrm{Mg}$ & -13.77847800 & -11.59591400 & $\begin{array}{ll}0 & 0.00000000\end{array}$ \\
\hline $\mathrm{Cl}$ & -12.72747800 & -9.77491400 & 1.36300000 \\
\hline $\mathrm{Cl}$ & -11.67547800 & -11.59591400 & -1.36300000 \\
\hline $\mathrm{Mg}$ & -13.77847800 & -7.95491400 & 0.00000000 \\
\hline $\mathrm{Cl}$ & -12.72747800 & -6.13391400 & 1.36300000 \\
\hline $\mathrm{Cl}$ & -11.67547800 & -7.95491400 & -1.36300000 \\
\hline $\mathrm{Mg}$ & -13.77847800 & -4.31391400 & 0.00000000 \\
\hline $\mathrm{Cl}$ & -12.72747800 & -2.49291400 & 1.36300000 \\
\hline $\mathrm{Cl}$ & -11.67547800 & -4.31391400 & -1.36300000 \\
\hline $\mathrm{Mg}$ & -13.77847800 & -0.67291400 & 0.00000000 \\
\hline
\end{tabular}




\begin{tabular}{|c|c|c|}
\hline $\mathrm{Cl}$ & -12.72747800 & 1.36300000 \\
\hline $\mathrm{Cl}$ & $-11.67547800 \quad-0.67291400$ & -1.36300000 \\
\hline $\mathrm{Mg}$ & $-13.77847800 \quad 2.96808600$ & 0.00000000 \\
\hline $\mathrm{Cl}$ & $-12.72747800 \quad 4.78908600$ & 1.36300000 \\
\hline $\mathrm{Cl}$ & -11.67547800 & -1.36300000 \\
\hline $\mathrm{Mg}$ & -13.77847800 & 0.00000000 \\
\hline $\mathrm{Cl}$ & -12.72747800 & 1.36300000 \\
\hline $\mathrm{Cl}$ & -11.67547800 & -1.36300000 \\
\hline $\mathrm{Mg}$ & $-13.77847800 \quad 10.25008600$ & 0.00000000 \\
\hline $\mathrm{Cl}$ & $-12.72747800 \quad 12.07108600$ & 1.36300000 \\
\hline $\mathrm{Cl}$ & $-11.67547800 \quad 10.25008600$ & -1.36300000 \\
\hline $\mathrm{Mg}$ & $-13.77847800 \quad 13.89108600$ & 0.00000000 \\
\hline $\mathrm{Cl}$ & $-12.72747800 \quad 15.71208600$ & 1.36300000 \\
\hline $\mathrm{Cl}$ & $-11.67547800 \quad 13.89108600$ & -1.36300000 \\
\hline $\mathrm{Mg}$ & $-13.77847800 \quad 17.53208600$ & 0.00000000 \\
\hline $\mathrm{Cl}$ & $-12.72747800 \quad 19.35308600$ & 1.36300000 \\
\hline $\mathrm{Cl}$ & $-11.67547800 \quad 17.53208600$ & -1.36300000 \\
\hline $\mathrm{Mg}$ & $-13.77847800 \quad 21.17308600$ & 0.00000000 \\
\hline $\mathrm{Cl}$ & $-11.67547800 \quad 21.17308600$ & -1.36300000 \\
\hline $\mathrm{Cl}$ & $-9.57347800-15.23691400$ & 1.36300000 \\
\hline $\mathrm{Mg}$ & $-10.62447800-13.4169140$ & o 0.00000000 \\
\hline $\mathrm{Cl}$ & $-9.57347800-11.59591400$ & 1.36300000 \\
\hline $\mathrm{Cl}$ & $-8.52247800-13.41691400$ & -1.36300000 \\
\hline $\mathrm{Mg}$ & $-10.62447800 \quad-9.77491400$ & 0.00000000 \\
\hline $\mathrm{Cl}$ & $-9.57347800 \quad-7.95491400$ & 1.36300000 \\
\hline $\mathrm{Cl}$ & $-8.52247800 \quad-9.77491400$ & -1.36300000 \\
\hline $\mathrm{Mg}$ & $-10.62447800-6.13391400$ & 0.00000000 \\
\hline $\mathrm{Cl}$ & $-9.57347800 \quad-4.31391400$ & 1.36300000 \\
\hline $\mathrm{Cl}$ & $-8.52247800-6.13391400$ & -1.36300000 \\
\hline $\mathrm{Mg}$ & $-10.62447800-2.49291400$ & 0.00000000 \\
\hline $\mathrm{Cl}$ & $\begin{array}{ll}-9.57347800 & -0.67291400\end{array}$ & 1.36300000 \\
\hline $\mathrm{Cl}$ & $-8.52247800 \quad-2.49291400$ & -1.36300000 \\
\hline $\mathrm{Mg}$ & $-10.62447800 \quad 1.14808600$ & 0.00000000 \\
\hline $\mathrm{Cl}$ & $\begin{array}{ll}-9.57347800 & 2.96808600\end{array}$ & 1.36300000 \\
\hline $\mathrm{Cl}$ & -8.52247800 & -1.36300000 \\
\hline $\mathrm{Mg}$ & $\begin{array}{ll}-10.62447800 & 4.78908600\end{array}$ & 0.00000000 \\
\hline $\mathrm{Cl}$ & $-9.57347800 \quad 6.60908600$ & 1.36300000 \\
\hline $\mathrm{Cl}$ & $-8.52247800 \quad 4.78908600$ & -1.36300000 \\
\hline $\mathrm{Mg}$ & $-10.62447800 \quad 8.43008600$ & 0.00000000 \\
\hline $\mathrm{Cl}$ & $\begin{array}{ll}-9.57347800 & 10.25008600\end{array}$ & 1.36300000 \\
\hline $\mathrm{Cl}$ & $-8.52247800 \quad 8.43008600$ & -1.36300000 \\
\hline $\mathrm{Mg}$ & $-10.62447800 \quad 12.07108600$ & 0.00000000 \\
\hline $\mathrm{Cl}$ & $-9.57347800 \quad 13.89108600$ & 1.36300000 \\
\hline $\mathrm{Cl}$ & $-8.52247800 \quad 12.07108600$ & -1.36300000 \\
\hline $\mathrm{Mg}$ & $-10.62447800 \quad 15.71208600$ & 0.00000000 \\
\hline $\mathrm{Cl}$ & $\begin{array}{ll}-9.57347800 & 17.53208600\end{array}$ & 1.36300000 \\
\hline $\mathrm{Cl}$ & $-8.52247800 \quad 15.71208600$ & -1.36300000 \\
\hline $\mathrm{Mg}$ & $-10.62447800 \quad 19.35308600$ & 0.00000000 \\
\hline $\mathrm{Cl}$ & $-8.52247800 \quad 19.35308600$ & -1.36300000 \\
\hline $\mathrm{Cl}$ & $-6.42047800-17.05791400$ & 1.36300000 \\
\hline $\mathrm{Mg}$ & $-7.47147800-15.23691400$ & 0.00000000 \\
\hline $\mathrm{Cl}$ & $-6.42047800-13.41691400$ & 1.36300000 \\
\hline $\mathrm{Cl}$ & $\begin{array}{lll}-5.36947800 & -15.23691400\end{array}$ & -1.36300000 \\
\hline $\mathrm{Mg}$ & $-7.47147800-11.59591400$ & 0.00000000 \\
\hline $\mathrm{Cl}$ & $\begin{array}{ll}-6.42047800 & -9.77491400\end{array}$ & 1.36300000 \\
\hline $\mathrm{Cl}$ & $\begin{array}{ll}-5.36947800 & -11.59591400\end{array}$ & -1.36300000 \\
\hline $\mathrm{Mg}$ & $-7.47147800 \quad-7.95491400$ & 0.00000000 \\
\hline $\mathrm{Cl}$ & $-6.42047800-6.13391400$ & 1.36300000 \\
\hline $\mathrm{Cl}$ & $\begin{array}{ll}-5.36947800 & -7.95491400\end{array}$ & -1.36300000 \\
\hline $\mathrm{Mg}$ & $-7.47147800 \quad-4.31391400$ & 0.00000000 \\
\hline $\mathrm{Cl}$ & $6.42047800 \quad-2.49291400$ & \\
\hline
\end{tabular}




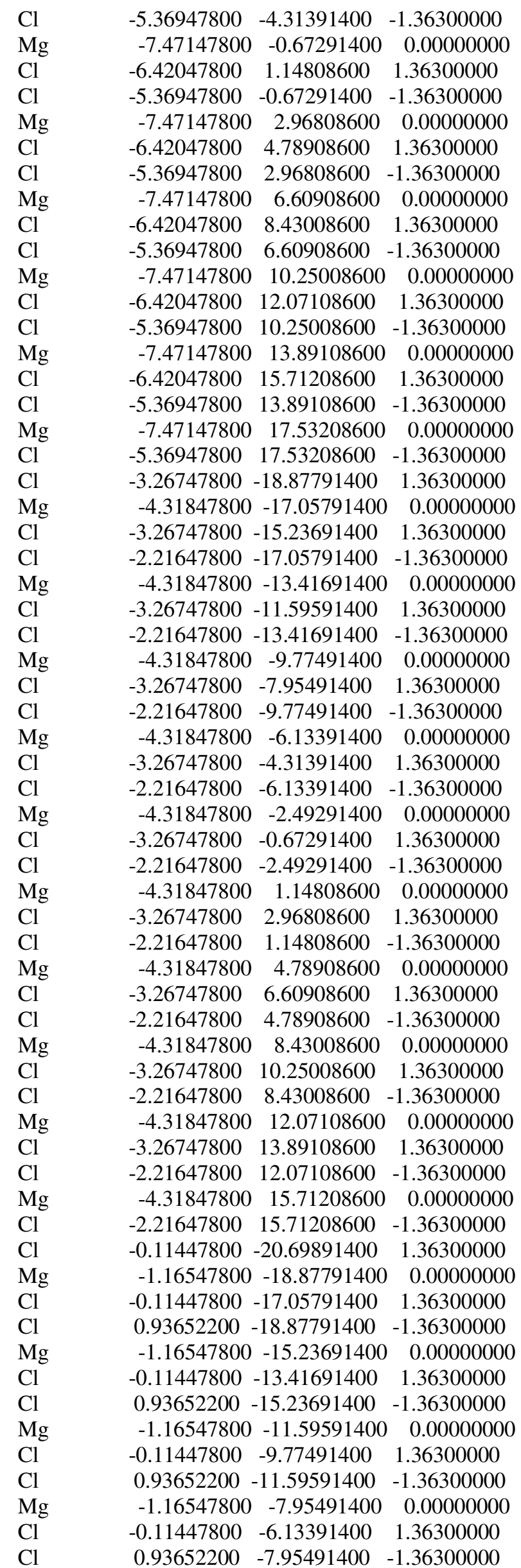




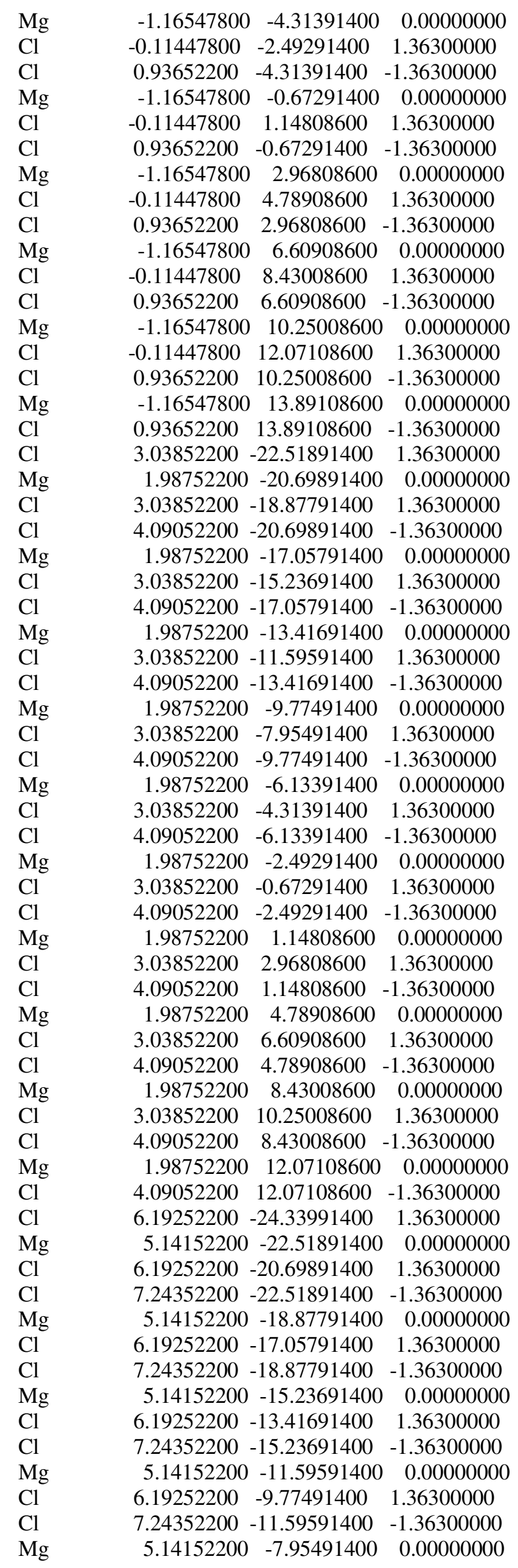




\begin{tabular}{|c|c|c|c|}
\hline $\mathrm{Cl}$ & 6.19252200 & -6.13391400 & 1.36300000 \\
\hline $\mathrm{Cl}$ & 7.24352200 & -7.95491400 & -1.36300000 \\
\hline $\mathrm{Mg}$ & 5.14152200 & -4.31391400 & 0.00000000 \\
\hline $\mathrm{Cl}$ & 6.19252200 & -2.49291400 & 1.36300000 \\
\hline $\mathrm{Cl}$ & 7.24352200 & -4.31391400 & -1.36300000 \\
\hline $\mathrm{Mg}$ & 5.14152200 & -0.67291400 & 0.00000000 \\
\hline $\mathrm{Cl}$ & 6.19252200 & 1.14808600 & 1.36300000 \\
\hline $\mathrm{Cl}$ & 7.24352200 & -0.67291400 & -1.36300000 \\
\hline $\mathrm{Mg}$ & 5.14152200 & 2.96808600 & 0.00000000 \\
\hline $\mathrm{Cl}$ & 6.19252200 & 4.78908600 & 1.36300000 \\
\hline $\mathrm{Cl}$ & 7.24352200 & 2.96808600 & -1.36300000 \\
\hline $\mathrm{Mg}$ & 5.14152200 & 6.60908600 & 0.00000000 \\
\hline $\mathrm{Cl}$ & 6.19252200 & 8.43008600 & 1.36300000 \\
\hline $\mathrm{Cl}$ & 7.24352200 & 6.60908600 & -1.36300000 \\
\hline $\mathrm{Mg}$ & 5.14152200 & 10.25008600 & 0.00000000 \\
\hline $\mathrm{Cl}$ & 7.24352200 & 10.25008600 & -1.36300000 \\
\hline $\mathrm{Cl}$ & $9.34552200-$ & -26.15991400 & 1.36300000 \\
\hline $\mathrm{Mg}$ & 8.29452200 & -24.33991400 & 0.00000000 \\
\hline $\mathrm{Cl}$ & $9.34552200-$ & -22.51891400 & 1.36300000 \\
\hline $\mathrm{Cl}$ & 10.39652200 & -24.33991400 & -1.36300000 \\
\hline $\mathrm{Mg}$ & 8.29452200 & -20.69891400 & 0.00000000 \\
\hline $\mathrm{Cl}$ & 9.34552200 & -18.87791400 & 1.36300000 \\
\hline $\mathrm{Cl}$ & 10.39652200 & -20.69891400 & -1.36300000 \\
\hline $\mathrm{Mg}$ & 8.29452200 & -17.05791400 & 0.00000000 \\
\hline $\mathrm{Cl}$ & 9.34552200 & -15.23691400 & 1.36300000 \\
\hline $\mathrm{Cl}$ & 10.39652200 & -17.05791400 & -1.36300000 \\
\hline $\mathrm{Mg}$ & 8.29452200 & -13.41691400 & 0.00000000 \\
\hline $\mathrm{Cl}$ & 9.34552200 & -11.59591400 & 1.36300000 \\
\hline $\mathrm{Cl}$ & 10.39652200 & -13.41691400 & -1.36300000 \\
\hline $\mathrm{Mg}$ & 8.29452200 & -9.77491400 & 0.00000000 \\
\hline $\mathrm{Cl}$ & 9.34552200 & -7.95491400 & 1.36300000 \\
\hline $\mathrm{Cl}$ & 10.39652200 & -9.77491400 & -1.36300000 \\
\hline $\mathrm{Mg}$ & 8.29452200 & -6.13391400 & 0.00000000 \\
\hline $\mathrm{Cl}$ & 9.34552200 & -4.31391400 & 1.36300000 \\
\hline $\mathrm{Cl}$ & 10.39652200 & -6.13391400 & -1.36300000 \\
\hline $\mathrm{Mg}$ & 8.29452200 & -2.49291400 & 0.00000000 \\
\hline $\mathrm{Cl}$ & 9.34552200 & -0.67291400 & 1.36300000 \\
\hline $\mathrm{Cl}$ & 10.39652200 & -2.49291400 & -1.36300000 \\
\hline $\mathrm{Mg}$ & 8.29452200 & 1.14808600 & 0.00000000 \\
\hline $\mathrm{Cl}$ & 9.34552200 & 2.96808600 & 1.36300000 \\
\hline $\mathrm{Cl}$ & 10.39652200 & 1.14808600 & -1.36300000 \\
\hline $\mathrm{Mg}$ & 8.29452200 & 4.78908600 & 0.00000000 \\
\hline $\mathrm{Cl}$ & 9.34552200 & 6.60908600 & 1.36300000 \\
\hline $\mathrm{Cl}$ & 10.39652200 & 4.78908600 & -1.36300000 \\
\hline $\mathrm{Mg}$ & 8.29452200 & 8.43008600 & 0.00000000 \\
\hline $\mathrm{Cl}$ & 10.39652200 & 8.43008600 & -1.36300000 \\
\hline $\mathrm{Cl}$ & 12.49852200 & -27.98091400 & 1.36300000 \\
\hline $\mathrm{Mg}$ & 11.44752200 & $\begin{array}{ll}0 & -26.15991400\end{array}$ & $\begin{array}{ll}0 & 0.00000000\end{array}$ \\
\hline $\mathrm{Cl}$ & 12.49852200 & -24.33991400 & 1.36300000 \\
\hline $\mathrm{Mg}$ & 11.44752200 & $\begin{array}{ll}0 & -22.51891400\end{array}$ & $\begin{array}{ll}0 & 0.00000000\end{array}$ \\
\hline $\mathrm{Cl}$ & 12.49852200 & -20.69891400 & 1.36300000 \\
\hline $\mathrm{Mg}$ & 11.44752200 & $0-18.87791400$ & $\begin{array}{ll}0 & 0.00000000\end{array}$ \\
\hline $\mathrm{Cl}$ & 12.49852200 & -17.05791400 & 1.36300000 \\
\hline $\mathrm{Mg}$ & 11.44752200 & $\begin{array}{ll}0 & -15.23691400\end{array}$ & $\begin{array}{ll}0 & 0.00000000\end{array}$ \\
\hline $\mathrm{Cl}$ & 12.49852200 & -13.41691400 & 1.36300000 \\
\hline $\mathrm{Mg}$ & 11.44752200 & $\begin{array}{ll}0 & -11.59591400\end{array}$ & $\begin{array}{ll}0 & 0.00000000\end{array}$ \\
\hline $\mathrm{Cl}$ & 12.49852200 & -9.77491400 & 1.36300000 \\
\hline $\mathrm{Mg}$ & 11.44752200 & $\begin{array}{ll}0 & -7.95491400\end{array}$ & 0.00000000 \\
\hline $\mathrm{Cl}$ & 12.49852200 & -6.13391400 & 1.36300000 \\
\hline $\mathrm{Mg}$ & 11.44752200 & $\begin{array}{ll}0 & -4.31391400\end{array}$ & 0.00000000 \\
\hline $\mathrm{Cl}$ & 12.49852200 & -2.49291400 & 1.36300000 \\
\hline
\end{tabular}




$\begin{array}{lccc}\mathrm{Mg} & 11.44752200 & -0.67291400 & 0.00000000 \\ \mathrm{Cl} & 12.49852200 & 1.14808600 & 1.36300000 \\ \mathrm{Mg} & 11.44752200 & 2.96808600 & 0.00000000 \\ \mathrm{Cl} & 12.49852200 & 4.78908600 & 1.36300000 \\ \mathrm{Mg} & 11.44752200 & 6.60908600 & 0.00000000\end{array}$

\section{$\mathrm{Mg}_{12} \mathrm{Cl}_{24}$ (truncated tetrahedron)}

Input keywords:

HF/gen opt iop(2/17=3) freq

Input coordinates:

$\begin{array}{lrrr}\mathrm{Mg} & 1.19675600 & 1.19675600 & -3.98413400 \\ \mathrm{Mg} & 3.98413400 & 1.19675600 & -1.19675600 \\ \mathrm{Mg} & -1.19675600 & -1.19675600 & -3.98413400 \\ \mathrm{Mg} & 1.19675600 & 3.98413400 & -1.19675600 \\ \mathrm{Mg} & 3.98413400 & -1.19675600 & 1.19675600 \\ \mathrm{Mg} & -3.98413400 & -1.19675600 & -1.19675600 \\ \mathrm{Mg} & -1.19675600 & -3.98413400 & -1.19675600 \\ \mathrm{Mg} & -1.19675600 & 3.98413400 & 1.19675600 \\ \mathrm{Mg} & 1.19675600 & -1.19675600 & 3.98413400 \\ \mathrm{Mg} & 1.19675600 & -3.98413400 & 1.19675600 \\ \mathrm{Mg} & -3.98413400 & 1.19675600 & 1.19675600 \\ \mathrm{Mg} & -1.19675600 & 1.19675600 & 3.98413400 \\ \mathrm{Cl} & 3.53888400 & 3.53888400 & -0.82422500 \\ \mathrm{Cl} & 0.82422500 & 3.53888400 & -3.53888400 \\ \mathrm{Cl} & 3.53888400 & 0.82422500 & -3.53888400 \\ \mathrm{Cl} & -3.53888400 & 0.82422500 & 3.53888400 \\ \mathrm{Cl} & -3.53888400 & 3.53888400 & 0.82422500 \\ \mathrm{Cl} & -0.82422500 & 3.53888400 & 3.53888400 \\ \mathrm{Cl} & 0.82422500 & -3.53888400 & 3.53888400 \\ \mathrm{Cl} & 3.53888400 & -0.82422500 & 3.53888400 \\ \mathrm{Cl} & 3.53888400 & -3.53888400 & 0.82422500 \\ \mathrm{Cl} & -3.53888400 & -0.82422500 & -3.53888400 \\ \mathrm{Cl} & -3.53888400 & -3.53888400 & -0.82422500 \\ \mathrm{Cl} & -0.82422500 & -3.53888400 & -3.53888400 \\ \mathrm{Cl} & 5.73400300 & 0.00000000 & 0.00000000 \\ \mathrm{Cl} & 0.00000000 & 0.00000000 & -5.73400300 \\ \mathrm{Cl} & 0.00000000 & -5.73400300 & 0.00000000 \\ \mathrm{Cl} & -5.73400300 & 0.00000000 & 0.00000000 \\ \mathrm{Cl} & 0.00000000 & 0.00000000 & 5.73400300 \\ \mathrm{Cl} & 0.00000000 & 5.73400300 & 0.00000000 \\ \mathrm{Cl} & 0.00000000 & 0.00000000 & 2.36667600 \\ \mathrm{Cl} & 0.00000000 & 2.36667600 & 0.00000000 \\ \mathrm{Cl} & 2.36667600 & 0.00000000 & 0.00000000 \\ \mathrm{Cl} & 0.00000000 & -2.36667600 & 0.00000000 \\ \mathrm{Cl} & 0.00000000 & 0.00000000 & -2.36667600 \\ \mathrm{Cl} & -2.36667600 & 0.00000000 & 0.00000000 \\ \mathrm{Ou} & \mathrm{y} & & \end{array}$

Output coordinates:

$\begin{array}{lrrr}\mathrm{Mg} & 1.205412 & 1.205412 & -4.007026 \\ \mathrm{Mg} & 4.007026 & 1.205412 & -1.205412 \\ \mathrm{Mg} & -1.205412 & -1.205412 & -4.007026 \\ \mathrm{Mg} & 1.205412 & 4.007026 & -1.205412 \\ \mathrm{Mg} & 4.007026 & -1.205412 & 1.205412 \\ \mathrm{Mg} & -4.007026 & -1.205412 & -1.205412 \\ \mathrm{Mg} & -1.205412 & -4.007026 & -1.205412 \\ \mathrm{Mg} & -1.205412 & 4.007026 & 1.205412 \\ \mathrm{Mg} & 1.205412 & -1.205412 & 4.007026 \\ \mathrm{Mg} & 1.205412 & -4.007026 & 1.205412 \\ \mathrm{Mg} & -4.007026 & 1.205412 & 1.205412 \\ \mathrm{Mg} & -1.205412 & 1.205412 & 4.007026\end{array}$




$\begin{array}{lrrr}\mathrm{Cl} & 3.547223 & 3.547223 & -0.865595 \\ \mathrm{Cl} & 0.865595 & 3.547223 & -3.547223 \\ \mathrm{Cl} & 3.547223 & 0.865595 & -3.547223 \\ \mathrm{Cl} & -3.547223 & 0.865595 & 3.547223 \\ \mathrm{Cl} & -3.547223 & 3.547223 & 0.865595 \\ \mathrm{Cl} & -0.865595 & 3.547223 & 3.547223 \\ \mathrm{Cl} & 0.865595 & -3.547223 & 3.547223 \\ \mathrm{Cl} & 3.547223 & -0.865595 & 3.547223 \\ \mathrm{Cl} & 3.547223 & -3.547223 & 0.865595 \\ \mathrm{Cl} & -3.547223 & -0.865595 & -3.547223 \\ \mathrm{Cl} & -3.547223 & -3.547223 & -0.865595 \\ \mathrm{Cl} & -0.865595 & -3.547223 & -3.547223 \\ \mathrm{Cl} & 5.748275 & 0.000000 & 0.000000 \\ \mathrm{Cl} & 0.000000 & 0.000000 & -5.748275 \\ \mathrm{Cl} & 0.000000 & -5.748275 & 0.000000 \\ \mathrm{Cl} & -5.748275 & 0.000000 & 0.000000 \\ \mathrm{Cl} & 0.000000 & 0.000000 & 5.748275 \\ \mathrm{Cl} & 0.000000 & 5.748275 & 0.000000 \\ \mathrm{Cl} & 0.000000 & 0.000000 & 2.416577 \\ \mathrm{Cl} & 0.000000 & 2.416577 & 0.000000 \\ \mathrm{Cl} & 2.416577 & 0.000000 & 0.000000 \\ \mathrm{Cl} & 0.000000 & -2.416577 & 0.000000 \\ \mathrm{Cl} & 0.000000 & 0.000000 & -2.416577 \\ \mathrm{Cl} & -2.416577 & 0.000000 & 0.000000\end{array}$

\section{$\mathrm{Mg}_{24} \mathrm{Cl}_{48}$ (truncated octahedron)}

Input keywords:

$\mathrm{HF} /$ gen opt freq

Input coordinates:

$\begin{array}{lrrr}\mathrm{Mg} & 0.00000000 & -2.92007400 & 5.21101500 \\ \mathrm{Mg} & -2.92007400 & 0.00000000 & 5.21101500 \\ \mathrm{Mg} & 0.00000000 & -5.21101500 & 2.92007400 \\ \mathrm{Mg} & 2.92007400 & 0.00000000 & 5.21101500 \\ \mathrm{Mg} & -5.21101500 & 0.00000000 & 2.92007400 \\ \mathrm{Mg} & 0.00000000 & 2.92007400 & 5.21101500 \\ \mathrm{Mg} & 2.92007400 & -5.21101500 & 0.00000000 \\ \mathrm{Mg} & -2.92007400 & -5.21101500 & 0.00000000 \\ \mathrm{Mg} & 5.21101500 & 0.00000000 & 2.92007400 \\ \mathrm{Mg} & -5.21101500 & 2.92007400 & 0.00000000 \\ \mathrm{Mg} & -5.21101500 & -2.92007400 & 0.00000000 \\ \mathrm{Mg} & 0.00000000 & 5.21101500 & 2.92007400 \\ \mathrm{Mg} & 0.00000000 & -5.21101500 & -2.92007400 \\ \mathrm{Mg} & 5.21101500 & -2.92007400 & 0.00000000 \\ \mathrm{Mg} & 5.21101500 & 2.92007400 & 0.00000000 \\ \mathrm{Mg} & -5.21101500 & 0.00000000 & -2.92007400 \\ \mathrm{Mg} & -2.92007400 & 5.21101500 & 0.00000000 \\ \mathrm{Mg} & 2.92007400 & 5.21101500 & 0.00000000 \\ \mathrm{Mg} & 0.00000000 & -2.92007400 & -5.21101500 \\ \mathrm{Mg} & 5.21101500 & 0.00000000 & -2.92007400 \\ \mathrm{Mg} & -2.92007400 & 0.00000000 & -5.21101500 \\ \mathrm{Mg} & 0.00000000 & 5.21101500 & -2.92007400 \\ \mathrm{Mg} & 2.92007400 & 0.00000000 & -5.21101500 \\ \mathrm{Mg} & 0.00000000 & 2.92007400 & -5.21101500 \\ \mathrm{Cl} & -1.95032000 & -1.95032000 & 6.15257500 \\ \mathrm{Cl} & -1.95032000 & -6.15257500 & -1.95032000 \\ \mathrm{Cl} & -6.15257500 & 1.95032000 & 1.95032000 \\ \mathrm{Cl} & -1.95032000 & 6.15257500 & 1.95032000 \\ \mathrm{Cl} & 1.95032000 & 1.95032000 & 6.15257500 \\ \mathrm{Cl} & 1.95032000 & 6.15257500 & -1.95032000\end{array}$




$\begin{array}{lrrr}\mathrm{Cl} & 1.95032000 & -1.95032000 & 6.15257500 \\ \mathrm{Cl} & 6.15257500 & -1.95032000 & 1.95032000 \\ \mathrm{Cl} & 6.15257500 & 1.95032000 & 1.95032000 \\ \mathrm{Cl} & 1.95032000 & 6.15257500 & 1.95032000 \\ \mathrm{Cl} & -1.95032000 & 6.15257500 & -1.95032000 \\ \mathrm{Cl} & 1.95032000 & 1.95032000 & -6.15257500 \\ \mathrm{Cl} & -1.95032000 & 1.95032000 & -6.15257500 \\ \mathrm{Cl} & -6.15257500 & -1.95032000 & -1.95032000 \\ \mathrm{Cl} & -6.15257500 & 1.95032000 & -1.95032000 \\ \mathrm{Cl} & -1.95032000 & 1.95032000 & 6.15257500 \\ \mathrm{Cl} & -1.95032000 & -6.15257500 & 1.95032000 \\ \mathrm{Cl} & 1.95032000 & -6.15257500 & 1.95032000 \\ \mathrm{Cl} & 1.95032000 & -6.15257500 & -1.95032000 \\ \mathrm{Cl} & -1.95032000 & -1.95032000 & -6.15257500 \\ \mathrm{Cl} & 1.95032000 & -1.95032000 & -6.15257500 \\ \mathrm{Cl} & 6.15257500 & 1.95032000 & -1.95032000 \\ \mathrm{Cl} & 6.15257500 & -1.95032000 & -1.95032000 \\ \mathrm{Cl} & -6.15257500 & -1.95032000 & 1.95032000 \\ \mathrm{Cl} & 0.00000000 & 5.30507300 & 5.30507300 \\ \mathrm{Cl} & 5.30507300 & 5.30507300 & 0.00000000 \\ \mathrm{Cl} & 5.30507300 & 0.00000000 & 5.30507300 \\ \mathrm{Cl} & 0.00000000 & -5.30507300 & 5.30507300 \\ \mathrm{Cl} & 5.30507300 & -5.30507300 & 0.00000000 \\ \mathrm{Cl} & 5.30507300 & 0.00000000 & -5.30507300 \\ \mathrm{Cl} & 0.00000000 & -5.30507300 & -5.30507300 \\ \mathrm{Cl} & -5.30507300 & -5.30507300 & 0.00000000 \\ \mathrm{Cl} & -5.30507300 & 0.00000000 & -5.30507300 \\ \mathrm{Cl} & 0.00000000 & 5.30507300 & -5.30507300 \\ \mathrm{Cl} & -5.30507300 & 5.30507300 & 0.00000000 \\ \mathrm{Cl} & -5.30507300 & 0.00000000 & 5.30507300 \\ \mathrm{Cl} & 2.83068500 & 0.00000000 & 2.83068500 \\ \mathrm{Cl} & 0.00000000 & -2.83068500 & 2.83068500 \\ \mathrm{Cl} & -2.83068500 & 0.00000000 & 2.83068500 \\ \mathrm{Cl} & 0.00000000 & 2.83068500 & 2.83068500 \\ \mathrm{Cl} & -2.83068500 & 0.00000000 & -2.83068500 \\ \mathrm{Cl} & 0.00000000 & -2.83068500 & -2.83068500 \\ \mathrm{Cl} & 2.83068500 & 0.00000000 & -2.83068500 \\ \mathrm{Cl} & 0.00000000 & 2.83068500 & -2.83068500 \\ \mathrm{Cl} & 2.83068500 & 2.83068500 & 0.00000000 \\ \mathrm{Cl} & 2.83068500 & -2.83068500 & 0.00000000 \\ \mathrm{Cl} & -2.83068500 & -2.83068500 & 0.00000000 \\ \mathrm{Cl} & -2.83068500 & 2.83068500 & 0.00000000 \\ \mathrm{O} \mathrm{H} & & & \\ \mathrm{Cl} & & & \end{array}$

Output coordinates:

$\begin{array}{lrrr}\mathrm{Mg} & 0.000000 & -2.935044 & 5.249232 \\ \mathrm{Mg} & -2.935044 & 0.000000 & 5.249232 \\ \mathrm{Mg} & 0.000000 & -5.249232 & 2.935044 \\ \mathrm{Mg} & 2.935044 & 0.000000 & 5.249232 \\ \mathrm{Mg} & -5.249232 & 0.000000 & 2.935044 \\ \mathrm{Mg} & 0.000000 & 2.935044 & 5.249232 \\ \mathrm{Mg} & 2.935044 & -5.249232 & 0.000000 \\ \mathrm{Mg} & -2.935044 & -5.249232 & 0.000000 \\ \mathrm{Mg} & 5.249232 & 0.000000 & 2.935044 \\ \mathrm{Mg} & -5.249232 & 2.935044 & 0.000000 \\ \mathrm{Mg} & -5.249232 & -2.935044 & 0.000000 \\ \mathrm{Mg} & 0.000000 & 5.249232 & 2.935044 \\ \mathrm{Mg} & 0.000000 & -5.249232 & -2.935044 \\ \mathrm{Mg} & 5.249232 & -2.935044 & 0.000000 \\ \mathrm{Mg} & 5.249232 & 2.935044 & 0.000000 \\ \mathrm{Mg} & -5.249232 & 0.000000 & -2.935044 \\ \mathrm{Mg} & -2.935044 & 5.249232 & 0.000000 \\ \mathrm{Mg} & 2.935044 & 5.249232 & 0.000000\end{array}$




\begin{tabular}{|c|c|c|c|}
\hline $\mathrm{Mg}$ & 0.000000 & -2.935044 & -5.249232 \\
\hline $\mathrm{Mg}$ & 5.249232 & 0.000000 & -2.935044 \\
\hline $\mathrm{Mg}$ & -2.935044 & 0.000000 & -5.249232 \\
\hline $\mathrm{Mg}$ & 0.000000 & 5.249232 & -2.935044 \\
\hline $\mathrm{Mg}$ & 2.935044 & 0.000000 & -5.249232 \\
\hline $\mathrm{Mg}$ & 0.000000 & 2.935044 & -5.249232 \\
\hline $\mathrm{Cl}$ & -1.929116 & -1.929116 & 6.191456 \\
\hline $\mathrm{Cl}$ & -1.929116 & -6.191456 & -1.929116 \\
\hline $\mathrm{Cl}$ & -6.191456 & 1.929116 & 1.929116 \\
\hline $\mathrm{Cl}$ & -1.929116 & 6.191456 & 1.929116 \\
\hline $\mathrm{Cl}$ & 1.929116 & 1.929116 & 6.191456 \\
\hline $\mathrm{Cl}$ & 1.929116 & 6.191456 & -1.929116 \\
\hline $\mathrm{Cl}$ & 1.929116 & -1.929116 & 6.191456 \\
\hline $\mathrm{Cl}$ & 6.191456 & -1.929116 & 1.929116 \\
\hline $\mathrm{Cl}$ & 6.191456 & 1.929116 & 1.929116 \\
\hline $\mathrm{Cl}$ & 1.929116 & 6.191456 & 1.929116 \\
\hline $\mathrm{Cl}$ & -1.929116 & 6.191456 & -1.929116 \\
\hline $\mathrm{Cl}$ & 1.929116 & 1.929116 & -6.191456 \\
\hline $\mathrm{Cl}$ & -1.929116 & 1.929116 & -6.191456 \\
\hline $\mathrm{Cl}$ & -6.191456 & -1.929116 & -1.929116 \\
\hline $\mathrm{Cl}$ & -6.191456 & 1.929116 & -1.929116 \\
\hline $\mathrm{Cl}$ & -1.929116 & 1.929116 & 6.191456 \\
\hline $\mathrm{Cl}$ & -1.929116 & -6.191456 & 1.929116 \\
\hline $\mathrm{Cl}$ & 1.929116 & -6.191456 & 1.929116 \\
\hline $\mathrm{Cl}$ & 1.929116 & -6.191456 & -1.929116 \\
\hline $\mathrm{Cl}$ & -1.929116 & -1.929116 & -6.191456 \\
\hline $\mathrm{Cl}$ & 1.929116 & -1.929116 & -6.191456 \\
\hline $\mathrm{Cl}$ & 6.191456 & 1.929116 & -1.929116 \\
\hline $\mathrm{Cl}$ & 6.191456 & -1.929116 & -1.929116 \\
\hline $\mathrm{Cl}$ & -6.191456 & -1.929116 & 1.929116 \\
\hline $\mathrm{Cl}$ & 0.000000 & 5.317455 & 5.317455 \\
\hline $\mathrm{Cl}$ & 5.317455 & 5.317455 & 0.000000 \\
\hline $\mathrm{Cl}$ & 5.317455 & 0.000000 & 5.317455 \\
\hline $\mathrm{Cl}$ & 0.000000 & -5.317455 & 5.317455 \\
\hline $\mathrm{Cl}$ & 5.317455 & -5.317455 & 0.000000 \\
\hline $\mathrm{Cl}$ & 5.317455 & 0.000000 & -5.317455 \\
\hline $\mathrm{Cl}$ & 0.000000 & -5.317455 & -5.317455 \\
\hline $\mathrm{Cl}$ & -5.317455 & -5.317455 & 0.000000 \\
\hline $\mathrm{Cl}$ & -5.317455 & 0.000000 & -5.317455 \\
\hline $\mathrm{Cl}$ & 0.000000 & 5.317455 & -5.317455 \\
\hline $\mathrm{Cl}$ & -5.317455 & 5.317455 & 0.000000 \\
\hline $\mathrm{Cl}$ & -5.317455 & 0.000000 & 5.317455 \\
\hline $\mathrm{Cl}$ & 2.873336 & 0.000000 & 2.873336 \\
\hline $\mathrm{Cl}$ & 0.000000 & -2.873336 & 2.873336 \\
\hline $\mathrm{Cl}$ & -2.873336 & 0.000000 & 2.873336 \\
\hline $\mathrm{Cl}$ & 0.000000 & 2.873336 & 2.873336 \\
\hline $\mathrm{Cl}$ & -2.873336 & 0.000000 & -2.873336 \\
\hline $\mathrm{Cl}$ & 0.000000 & -2.873336 & -2.873336 \\
\hline $\mathrm{Cl}$ & 2.873336 & 0.000000 & -2.873336 \\
\hline $\mathrm{Cl}$ & 0.000000 & 2.873336 & -2.873336 \\
\hline $\mathrm{Cl}$ & 2.873336 & 2.873336 & 0.000000 \\
\hline $\mathrm{Cl}$ & 2.873336 & -2.873336 & 0.000000 \\
\hline $\mathrm{Cl}$ & -2.873336 & -2.873336 & 0.000000 \\
\hline $\mathrm{Cl}$ & -2.873336 & 2.873336 & 0.000000 \\
\hline
\end{tabular}

\section{$\mathrm{Mg}_{24} \mathrm{Cl}_{48}$ (truncated cube)}

Input keywords:

HF/gen opt iop(2/17=3) freq Input coordinates: 


\begin{tabular}{|c|c|c|c|}
\hline $\mathrm{Mg}$ & 4.44237300 & -1.61926700 & 4.44237300 \\
\hline $\mathrm{Mg}$ & 4.44237300 & -4.44237300 & 1.61926700 \\
\hline $\mathrm{Mg}$ & 4.44237300 & 1.61926700 & 4.44237300 \\
\hline $\mathrm{Mg}$ & 1.61926700 & -4.44237300 & 4.44237300 \\
\hline $\mathrm{Mg}$ & 4.44237300 & -4.44237300 & -1.61926700 \\
\hline $\mathrm{Mg}$ & 1.61926700 & 4.44237300 & 4.44237300 \\
\hline $\mathrm{Mg}$ & 4.44237300 & 4.44237300 & 1.61926700 \\
\hline $\mathrm{Mg}$ & -1.61926700 & -4.44237300 & 4.44237300 \\
\hline $\mathrm{Mg}$ & 1.61926700 & -4.44237300 & -4.44237300 \\
\hline $\mathrm{Mg}$ & 4.44237300 & -1.61926700 & -4.44237300 \\
\hline $\mathrm{Mg}$ & -1.61926700 & 4.44237300 & 4.44237300 \\
\hline $\mathrm{Mg}$ & 4.44237300 & 4.44237300 & -1.61926700 \\
\hline $\mathrm{Mg}$ & -4.44237300 & -1.61926700 & 4.44237300 \\
\hline $\mathrm{Mg}$ & -4.44237300 & -4.44237300 & 1.61926700 \\
\hline $\mathrm{Mg}$ & -1.61926700 & -4.44237300 & -4.44237300 \\
\hline $\mathrm{Mg}$ & 4.44237300 & 1.61926700 & -4.44237300 \\
\hline $\mathrm{Mg}$ & -4.44237300 & 1.61926700 & 4.44237300 \\
\hline $\mathrm{Mg}$ & -4.44237300 & 4.44237300 & 1.61926700 \\
\hline $\mathrm{Mg}$ & 1.61926700 & 4.44237300 & -4.44237300 \\
\hline $\mathrm{Mg}$ & -4.44237300 & -4.44237300 & -1.61926700 \\
\hline $\mathrm{Mg}$ & -4.44237300 & -1.61926700 & -4.44237300 \\
\hline $\mathrm{Mg}$ & -4.44237300 & 4.44237300 & -1.61926700 \\
\hline $\mathrm{Mg}$ & -1.61926700 & 4.44237300 & -4.44237300 \\
\hline $\mathrm{Mg}$ & -4.44237300 & 1.61926700 & -4.44237300 \\
\hline $\mathrm{Cl}$ & 2.99930100 & -5.73485600 & -2.99930100 \\
\hline $\mathrm{Cl}$ & 5.73485600 & -2.99930100 & -2.99930100 \\
\hline $\mathrm{Cl}$ & 2.99930100 & -2.99930100 & -5.73485600 \\
\hline $\mathrm{Cl}$ & 2.99930100 & 2.99930100 & -5.73485600 \\
\hline $\mathrm{Cl}$ & 5.73485600 & 2.99930100 & -2.99930100 \\
\hline $\mathrm{Cl}$ & 2.99930100 & 5.73485600 & -2.99930100 \\
\hline $\mathrm{Cl}$ & 2.99930100 & 5.73485600 & 2.99930100 \\
\hline $\mathrm{Cl}$ & 5.73485600 & 2.99930100 & 2.99930100 \\
\hline $\mathrm{Cl}$ & 2.99930100 & 2.99930100 & 5.73485600 \\
\hline $\mathrm{Cl}$ & 2.99930100 & -2.99930100 & 5.73485600 \\
\hline $\mathrm{Cl}$ & 5.73485600 & -2.99930100 & 2.99930100 \\
\hline $\mathrm{Cl}$ & 2.99930100 & -5.73485600 & 2.99930100 \\
\hline $\mathrm{Cl}$ & -2.99930100 & -5.73485600 & -2.99930100 \\
\hline $\mathrm{Cl}$ & -2.99930100 & -2.99930100 & -5.73485600 \\
\hline $\mathrm{Cl}$ & -5.73485600 & -2.99930100 & -2.99930100 \\
\hline $\mathrm{Cl}$ & -5.73485600 & 2.99930100 & -2.99930100 \\
\hline $\mathrm{Cl}$ & -2.99930100 & 2.99930100 & -5.73485600 \\
\hline $\mathrm{Cl}$ & -2.99930100 & 5.73485600 & -2.99930100 \\
\hline $\mathrm{Cl}$ & -5.73485600 & 2.99930100 & 2.99930100 \\
\hline $\mathrm{Cl}$ & -2.99930100 & 5.73485600 & 2.99930100 \\
\hline $\mathrm{Cl}$ & -2.99930100 & 2.99930100 & 5.73485600 \\
\hline $\mathrm{Cl}$ & -5.73485600 & -2.99930100 & 2.99930100 \\
\hline $\mathrm{Cl}$ & -2.99930100 & -5.73485600 & 2.99930100 \\
\hline $\mathrm{Cl}$ & -2.99930100 & -2.99930100 & 5.73485600 \\
\hline $\mathrm{Cl}$ & 0.00000000 & -5.68146200 & 5.68146200 \\
\hline $\mathrm{Cl}$ & 5.68146200 & -5.68146200 & 0.00000000 \\
\hline $\mathrm{Cl}$ & 0.00000000 & -5.68146200 & -5.68146200 \\
\hline $\mathrm{Cl}$ & -5.68146200 & -5.68146200 & 0.00000000 \\
\hline $\mathrm{Cl}$ & 5.68146200 & 0.00000000 & 5.68146200 \\
\hline $\mathrm{Cl}$ & -5.68146200 & 0.00000000 & 5.68146200 \\
\hline $\mathrm{Cl}$ & -5.68146200 & 0.00000000 & -5.68146200 \\
\hline $\mathrm{Cl}$ & 5.68146200 & 0.00000000 & -5.68146200 \\
\hline $\mathrm{Cl}$ & 0.00000000 & 5.68146200 & -5.68146200 \\
\hline $\mathrm{Cl}$ & -5.68146200 & 5.68146200 & 0.00000000 \\
\hline $\mathrm{Cl}$ & 0.00000000 & 5.68146200 & 5.68146200 \\
\hline $\mathrm{Cl}$ & 5.68146200 & 5.68146200 & 0.00000000 \\
\hline $\mathrm{Cl}$ & 3.20442900 & 0.00000000 & 3.20442900 \\
\hline
\end{tabular}




\begin{tabular}{|c|c|c|c|}
\hline $\mathrm{Cl}$ & 3.20442900 & 3.20442900 & 0.00000000 \\
\hline $\mathrm{Cl}$ & 3.20442900 & 0.00000000 & -3.20442900 \\
\hline $\mathrm{Cl}$ & 3.20442900 & -3.20442900 & 0.00000000 \\
\hline $\mathrm{Cl}$ & -3.20442900 & -3.20442900 & 0.00000000 \\
\hline $\mathrm{Cl}$ & -3.20442900 & 0.00000000 & -3.20442900 \\
\hline $\mathrm{Cl}$ & -3.20442900 & 3.20442900 & 0.00000000 \\
\hline $\mathrm{Cl}$ & -3.20442900 & 0.00000000 & 3.20442900 \\
\hline $\mathrm{Cl}$ & 0.00000000 & -3.20442900 & 3.20442900 \\
\hline $\mathrm{Cl}$ & 0.00000000 & -3.20442900 & -3.20442900 \\
\hline $\mathrm{Cl}$ & 0.00000000 & 3.20442900 & -3.20442900 \\
\hline $\mathrm{Cl}$ & 0.00000000 & 3.20442900 & 3.20442900 \\
\hline
\end{tabular}

Output coordinates:

\begin{tabular}{|c|c|c|c|}
\hline $\mathrm{Mg}$ & 4.446965 & -1.632734 & 4.446965 \\
\hline $\mathrm{Mg}$ & 4.446965 & -4.446965 & 1.632734 \\
\hline $\mathrm{Mg}$ & 4.446965 & 1.632734 & 4.446965 \\
\hline $\mathrm{Mg}$ & 1.632734 & -4.446965 & 4.446965 \\
\hline $\mathrm{Mg}$ & 4.446965 & -4.446965 & -1.632734 \\
\hline $\mathrm{Mg}$ & 1.632734 & 4.446965 & 4.446965 \\
\hline $\mathrm{Mg}$ & 4.446965 & 4.446965 & 1.632734 \\
\hline $\mathrm{Mg}$ & -1.632734 & -4.446965 & 4.446965 \\
\hline $\mathrm{Mg}$ & 1.632734 & -4.446965 & -4.446965 \\
\hline $\mathrm{Mg}$ & 4.446965 & -1.632734 & -4.446965 \\
\hline $\mathrm{Mg}$ & -1.632734 & 4.446965 & 4.446965 \\
\hline $\mathrm{Mg}$ & 4.446965 & 4.446965 & -1.632734 \\
\hline $\mathrm{Mg}$ & -4.446965 & -1.632734 & 4.446965 \\
\hline $\mathrm{Mg}$ & -4.446965 & -4.446965 & 1.632734 \\
\hline $\mathrm{Mg}$ & -1.632734 & -4.446965 & -4.446965 \\
\hline $\mathrm{Mg}$ & 4.446965 & 1.632734 & -4.446965 \\
\hline $\mathrm{Mg}$ & -4.446965 & 1.632734 & 4.446965 \\
\hline $\mathrm{Mg}$ & -4.446965 & 4.446965 & 1.632734 \\
\hline $\mathrm{Mg}$ & 1.632734 & 4.446965 & -4.446965 \\
\hline $\mathrm{Mg}$ & -4.446965 & -4.446965 & -1.632734 \\
\hline $\mathrm{Mg}$ & -4.446965 & -1.632734 & -4.446965 \\
\hline $\mathrm{Mg}$ & -4.446965 & 4.446965 & -1.632734 \\
\hline $\mathrm{Mg}$ & -1.632734 & 4.446965 & -4.446965 \\
\hline $\mathrm{Mg}$ & -4.446965 & 5 1.632734 & -4.446965 \\
\hline $\mathrm{Cl}$ & 3.022525 & -5.733469 & -3.022525 \\
\hline $\mathrm{Cl}$ & 5.733469 & -3.022525 & -3.022525 \\
\hline $\mathrm{Cl}$ & 3.022525 & -3.022525 & -5.733469 \\
\hline $\mathrm{Cl}$ & 3.022525 & 3.022525 & -5.733469 \\
\hline $\mathrm{Cl}$ & 5.733469 & 3.022525 & -3.022525 \\
\hline $\mathrm{Cl}$ & 3.022525 & 5.733469 & -3.022525 \\
\hline $\mathrm{Cl}$ & 3.022525 & 5.733469 & 3.022525 \\
\hline $\mathrm{Cl}$ & 5.733469 & 3.022525 & 3.022525 \\
\hline $\mathrm{Cl}$ & 3.022525 & 3.022525 & 5.733469 \\
\hline $\mathrm{Cl}$ & 3.022525 & -3.022525 & 5.733469 \\
\hline $\mathrm{Cl}$ & 5.733469 & -3.022525 & 3.022525 \\
\hline $\mathrm{Cl}$ & 3.022525 & -5.733469 & 3.022525 \\
\hline $\mathrm{Cl}$ & -3.022525 & -5.733469 & -3.022525 \\
\hline $\mathrm{Cl}$ & -3.022525 & -3.022525 & -5.733469 \\
\hline $\mathrm{Cl}$ & -5.733469 & -3.022525 & -3.022525 \\
\hline $\mathrm{Cl}$ & -5.733469 & 3.022525 & -3.022525 \\
\hline $\mathrm{Cl}$ & -3.022525 & 3.022525 & -5.733469 \\
\hline $\mathrm{Cl}$ & -3.022525 & 5.733469 & -3.022525 \\
\hline $\mathrm{Cl}$ & -5.733469 & 3.022525 & 3.022525 \\
\hline $\mathrm{Cl}$ & -3.022525 & 5.733469 & 3.022525 \\
\hline $\mathrm{Cl}$ & -3.022525 & 3.022525 & 5.733469 \\
\hline $\mathrm{Cl}$ & -5.733469 & -3.022525 & 3.022525 \\
\hline $\mathrm{Cl}$ & -3.022525 & -5.733469 & 3.022525 \\
\hline $\mathrm{Cl}$ & -3.022525 & -3.022525 & 5.733469 \\
\hline $\mathrm{Cl}$ & 0.000000 & -5.672853 & 5.672853 \\
\hline
\end{tabular}




$\begin{array}{lrrr}\mathrm{Cl} & 5.672853 & -5.672853 & 0.000000 \\ \mathrm{Cl} & 0.000000 & -5.672853 & -5.672853 \\ \mathrm{Cl} & -5.672853 & -5.672853 & 0.000000 \\ \mathrm{Cl} & 5.672853 & 0.000000 & 5.672853 \\ \mathrm{Cl} & -5.672853 & 0.000000 & 5.672853 \\ \mathrm{Cl} & -5.672853 & 0.000000 & -5.672853 \\ \mathrm{Cl} & 5.672853 & 0.000000 & -5.672853 \\ \mathrm{Cl} & 0.000000 & 5.672853 & -5.672853 \\ \mathrm{Cl} & -5.672853 & 5.672853 & 0.000000 \\ \mathrm{Cl} & 0.000000 & 5.672853 & 5.672853 \\ \mathrm{Cl} & 5.672853 & 5.672853 & 0.000000 \\ \mathrm{Cl} & 3.223380 & 0.000000 & 3.223380 \\ \mathrm{Cl} & 3.223380 & 3.223380 & 0.000000 \\ \mathrm{Cl} & 3.223380 & 0.000000 & -3.223380 \\ \mathrm{Cl} & 3.223380 & -3.223380 & 0.000000 \\ \mathrm{Cl} & -3.223380 & -3.223380 & 0.000000 \\ \mathrm{Cl} & -3.223380 & 0.000000 & -3.223380 \\ \mathrm{Cl} & -3.223380 & 3.223380 & 0.000000 \\ \mathrm{Cl} & -3.223380 & 0.000000 & 3.223380 \\ \mathrm{Cl} & 0.000000 & -3.223380 & 3.223380 \\ \mathrm{Cl} & 0.000000 & -3.223380 & -3.223380 \\ \mathrm{Cl} & 0.000000 & 3.223380 & -3.223380 \\ \mathrm{Cl} & 0.000000 & 3.223380 & 3.223380\end{array}$

\section{$\mathrm{Mg}_{48} \mathrm{Cl}_{96}$ (truncated cuboctahedron)}

Input keywords:

$\begin{array}{lrrr}\mathrm{HF} / \mathrm{gen} \text { opt freq=numer } & & \\ \mathrm{Input} \text { coordinates: } & & \\ \mathrm{Mg} & 1.63005900 & -7.33020400 & -4.48782200 \\ \mathrm{Mg} & -1.63005900 & -7.33020400 & -4.48782200 \\ \mathrm{Mg} & 1.63005900 & -4.48782200 & -7.33020400 \\ \mathrm{Mg} & 4.48782200 & -7.33020400 & -1.63005900 \\ \mathrm{Mg} & -1.63005900 & -4.48782200 & -7.33020400 \\ \mathrm{Mg} & -4.48782200 & -7.33020400 & -1.63005900 \\ \mathrm{Mg} & 4.48782200 & -1.63005900 & -7.33020400 \\ \mathrm{Mg} & 4.48782200 & -7.33020400 & 1.63005900 \\ \mathrm{Mg} & 7.33020400 & -4.48782200 & -1.63005900 \\ \mathrm{Mg} & -4.48782200 & -1.63005900 & -7.33020400 \\ \mathrm{Mg} & -4.48782200 & -7.33020400 & 1.63005900 \\ \mathrm{Mg} & -7.33020400 & -4.48782200 & -1.63005900 \\ \mathrm{Mg} & 4.48782200 & 1.63005900 & -7.33020400 \\ \mathrm{Mg} & 7.33020400 & -1.63005900 & -4.48782200 \\ \mathrm{Mg} & 7.33020400 & -4.48782200 & 1.63005900 \\ \mathrm{Mg} & 1.63005900 & -7.33020400 & 4.48782200 \\ \mathrm{Mg} & -4.48782200 & 1.63005900 & -7.33020400 \\ \mathrm{Mg} & -7.33020400 & -1.63005900 & -4.48782200 \\ \mathrm{Mg} & -7.33020400 & -4.48782200 & 1.63005900 \\ \mathrm{Mg} & -1.63005900 & -7.33020400 & 4.48782200 \\ \mathrm{Mg} & 7.33020400 & 1.63005900 & -4.48782200 \\ \mathrm{Mg} & 1.63005900 & 4.48782200 & -7.33020400 \\ \mathrm{Mg} & 7.33020400 & -1.63005900 & 4.48782200 \\ \mathrm{Mg} & 1.63005900 & -4.48782200 & 7.33020400 \\ \mathrm{Mg} & -7.33020400 & 1.63005900 & -4.48782200 \\ \mathrm{Mg} & -1.63005900 & 4.48782200 & -7.33020400 \\ \mathrm{Mg} & -7.33020400 & -1.63005900 & 4.48782200 \\ \mathrm{Mg} & -1.63005900 & -4.48782200 & 7.33020400 \\ \mathrm{Mg} & 7.33020400 & 4.48782200 & -1.63005900 \\ \mathrm{Mg} & 1.63005900 & 7.33020400 & -4.48782200 \\ \mathrm{Mg} & 7.33020400 & 1.63005900 & 4.48782200\end{array}$




\begin{tabular}{|c|c|c|c|}
\hline $\mathrm{Mg}$ & 4.48782200 & -1.63005900 & 7.33020400 \\
\hline $\mathrm{Mg}$ & -7.33020400 & 4.48782200 & -1.63005900 \\
\hline $\mathrm{Mg}$ & -1.63005900 & 7.33020400 & -4.48782200 \\
\hline $\mathrm{Mg}$ & -7.33020400 & 1.63005900 & 4.48782200 \\
\hline $\mathrm{Mg}$ & -4.48782200 & -1.63005900 & 7.33020400 \\
\hline $\mathrm{Mg}$ & 7.33020400 & 4.48782200 & 1.63005900 \\
\hline $\mathrm{Mg}$ & 4.48782200 & 7.33020400 & -1.63005900 \\
\hline $\mathrm{Mg}$ & 4.48782200 & 1.63005900 & 7.33020400 \\
\hline $\mathrm{Mg}$ & -7.33020400 & 4.48782200 & 1.63005900 \\
\hline $\mathrm{Mg}$ & -4.48782200 & 7.33020400 & -1.63005900 \\
\hline $\mathrm{Mg}$ & -4.48782200 & 1.63005900 & 7.33020400 \\
\hline $\mathrm{Mg}$ & 4.48782200 & 7.33020400 & 1.63005900 \\
\hline $\mathrm{Mg}$ & 1.63005900 & 4.48782200 & 7.33020400 \\
\hline $\mathrm{Mg}$ & -4.48782200 & 7.33020400 & 1.63005900 \\
\hline $\mathrm{Mg}$ & -1.63005900 & 4.48782200 & 7.33020400 \\
\hline $\mathrm{Mg}$ & 1.63005900 & 7.33020400 & 4.48782200 \\
\hline $\mathrm{Mg}$ & -1.63005900 & 7.33020400 & 4.48782200 \\
\hline $\mathrm{Cl}$ & 3.09322700 & 3.09322700 & 8.57051000 \\
\hline $\mathrm{Cl}$ & 3.28052200 & 0.00000000 & 6.06768200 \\
\hline $\mathrm{Cl}$ & 3.09322700 & -3.09322700 & 8.57051000 \\
\hline $\mathrm{Cl}$ & 0.00000000 & -3.28052200 & 6.06768200 \\
\hline $\mathrm{Cl}$ & -3.09322700 & -3.09322700 & 8.57051000 \\
\hline $\mathrm{Cl}$ & -3.28052200 & 0.00000000 & 6.06768200 \\
\hline $\mathrm{Cl}$ & -3.09322700 & 3.09322700 & 8.57051000 \\
\hline $\mathrm{Cl}$ & 0.00000000 & 3.28052200 & 6.06768200 \\
\hline $\mathrm{Cl}$ & 3.09322700 & 8.57051000 & 3.09322700 \\
\hline $\mathrm{Cl}$ & 0.00000000 & 6.06768200 & 3.28052200 \\
\hline $\mathrm{Cl}$ & -3.09322700 & 8.57051000 & 3.09322700 \\
\hline $\mathrm{Cl}$ & -3.28052200 & 6.06768200 & 0.00000000 \\
\hline $\mathrm{Cl}$ & -3.09322700 & 8.57051000 & -3.09322700 \\
\hline $\mathrm{Cl}$ & 0.00000000 & 6.06768200 & -3.28052200 \\
\hline $\mathrm{Cl}$ & 3.09322700 & 8.57051000 & -3.09322700 \\
\hline $\mathrm{Cl}$ & 3.28052200 & 6.06768200 & 0.00000000 \\
\hline $\mathrm{Cl}$ & 6.06768200 & 0.00000000 & 3.28052200 \\
\hline $\mathrm{Cl}$ & 8.57051000 & 3.09322700 & 3.09322700 \\
\hline $\mathrm{Cl}$ & 6.06768200 & 3.28052200 & 0.00000000 \\
\hline $\mathrm{Cl}$ & 8.57051000 & 3.09322700 & -3.09322700 \\
\hline $\mathrm{Cl}$ & 6.06768200 & 0.00000000 & -3.28052200 \\
\hline $\mathrm{Cl}$ & 8.57051000 & -3.09322700 & -3.09322700 \\
\hline $\mathrm{Cl}$ & 6.06768200 & -3.28052200 & 0.00000000 \\
\hline $\mathrm{Cl}$ & 8.57051000 & -3.09322700 & 3.09322700 \\
\hline $\mathrm{Cl}$ & 3.28052200 & -6.06768200 & 0.00000000 \\
\hline $\mathrm{Cl}$ & 3.09322700 & -8.57051000 & -3.09322700 \\
\hline $\mathrm{Cl}$ & 0.00000000 & -6.06768200 & -3.28052200 \\
\hline $\mathrm{Cl}$ & -3.09322700 & -8.57051000 & -3.09322700 \\
\hline $\mathrm{Cl}$ & -3.28052200 & -6.06768200 & 0.00000000 \\
\hline $\mathrm{Cl}$ & -3.09322700 & -8.57051000 & 3.09322700 \\
\hline $\mathrm{Cl}$ & 0.00000000 & -6.06768200 & 3.28052200 \\
\hline $\mathrm{Cl}$ & 3.09322700 & -8.57051000 & 3.09322700 \\
\hline $\mathrm{Cl}$ & -8.57051000 & -3.09322700 & 3.09322700 \\
\hline $\mathrm{Cl}$ & -6.06768200 & -3.28052200 & 0.00000000 \\
\hline $\mathrm{Cl}$ & -8.57051000 & -3.09322700 & -3.09322700 \\
\hline $\mathrm{Cl}$ & -6.06768200 & 0.00000000 & -3.28052200 \\
\hline $\mathrm{Cl}$ & -8.57051000 & 3.09322700 & -3.09322700 \\
\hline $\mathrm{Cl}$ & -6.06768200 & 3.28052200 & 0.00000000 \\
\hline $\mathrm{Cl}$ & -8.57051000 & 3.09322700 & 3.09322700 \\
\hline $\mathrm{Cl}$ & -6.06768200 & 0.00000000 & 3.28052200 \\
\hline $\mathrm{Cl}$ & -3.28052200 & 0.00000000 & -6.06768200 \\
\hline $\mathrm{Cl}$ & -3.09322700 & -3.09322700 & -8.57051000 \\
\hline $\mathrm{Cl}$ & 0.00000000 & -3.28052200 & -6.06768200 \\
\hline $\mathrm{Cl}$ & 3.09322700 & -3.09322700 & -8.57051000 \\
\hline
\end{tabular}




\begin{tabular}{|c|c|c|c|}
\hline $\mathrm{Cl}$ & 3.28052200 & 0.00000000 & -6.06768200 \\
\hline $\mathrm{Cl}$ & 3.09322700 & 3.09322700 & -8.57051000 \\
\hline $\mathrm{Cl}$ & 0.00000000 & 3.28052200 & -6.06768200 \\
\hline $\mathrm{Cl}$ & -3.09322700 & 3.09322700 & -8.57051000 \\
\hline $\mathrm{Cl}$ & -5.94263700 & 2.89229800 & -5.94263700 \\
\hline $\mathrm{Cl}$ & -5.94263700 & -2.89229800 & -5.94263700 \\
\hline $\mathrm{Cl}$ & -5.94263700 & -5.94263700 & -2.89229800 \\
\hline $\mathrm{Cl}$ & -5.94263700 & -5.94263700 & 2.89229800 \\
\hline $\mathrm{Cl}$ & -5.94263700 & -2.89229800 & 5.94263700 \\
\hline $\mathrm{Cl}$ & -5.94263700 & 2.89229800 & 5.94263700 \\
\hline $\mathrm{Cl}$ & -5.94263700 & 5.94263700 & 2.89229800 \\
\hline $\mathrm{Cl}$ & -5.94263700 & 5.94263700 & -2.89229800 \\
\hline $\mathrm{Cl}$ & 5.94263700 & -2.89229800 & 5.94263700 \\
\hline $\mathrm{Cl}$ & 5.94263700 & -5.94263700 & 2.89229800 \\
\hline $\mathrm{Cl}$ & 5.94263700 & -5.94263700 & -2.89229800 \\
\hline $\mathrm{Cl}$ & 5.94263700 & -2.89229800 & -5.94263700 \\
\hline $\mathrm{Cl}$ & 5.94263700 & 2.89229800 & -5.94263700 \\
\hline $\mathrm{Cl}$ & 5.94263700 & 5.94263700 & -2.89229800 \\
\hline $\mathrm{Cl}$ & 5.94263700 & 5.94263700 & 2.89229800 \\
\hline $\mathrm{Cl}$ & 5.94263700 & 2.89229800 & 5.94263700 \\
\hline $\mathrm{Cl}$ & 2.89229800 & 5.94263700 & -5.94263700 \\
\hline $\mathrm{Cl}$ & -2.89229800 & 5.94263700 & -5.94263700 \\
\hline $\mathrm{Cl}$ & -2.89229800 & -5.94263700 & -5.94263700 \\
\hline $\mathrm{Cl}$ & 2.89229800 & -5.94263700 & -5.94263700 \\
\hline $\mathrm{Cl}$ & 2.89229800 & -5.94263700 & 5.94263700 \\
\hline $\mathrm{Cl}$ & -2.89229800 & -5.94263700 & 5.94263700 \\
\hline $\mathrm{Cl}$ & -2.89229800 & 5.94263700 & 5.94263700 \\
\hline $\mathrm{Cl}$ & 2.89229800 & 5.94263700 & 5.94263700 \\
\hline $\mathrm{Cl}$ & 5.64124500 & -8.64078800 & 0.00000000 \\
\hline $\mathrm{Cl}$ & 8.64078800 & -5.64124500 & 0.00000000 \\
\hline $\mathrm{Cl}$ & 0.00000000 & -8.64078800 & -5.64124500 \\
\hline $\mathrm{Cl}$ & 0.00000000 & -5.64124500 & -8.64078800 \\
\hline $\mathrm{Cl}$ & -5.64124500 & -8.64078800 & 0.00000000 \\
\hline $\mathrm{Cl}$ & -8.64078800 & -5.64124500 & 0.00000000 \\
\hline $\mathrm{Cl}$ & 0.00000000 & -5.64124500 & 8.64078800 \\
\hline $\mathrm{Cl}$ & 0.00000000 & -8.64078800 & 5.64124500 \\
\hline $\mathrm{Cl}$ & -5.64124500 & 0.00000000 & -8.64078800 \\
\hline $\mathrm{Cl}$ & -8.64078800 & 0.00000000 & -5.64124500 \\
\hline $\mathrm{Cl}$ & -5.64124500 & 8.64078800 & 0.00000000 \\
\hline $\mathrm{Cl}$ & -8.64078800 & 5.64124500 & 0.00000000 \\
\hline $\mathrm{Cl}$ & -5.64124500 & 0.00000000 & 8.64078800 \\
\hline $\mathrm{Cl}$ & -8.64078800 & 0.00000000 & 5.64124500 \\
\hline $\mathrm{Cl}$ & 0.00000000 & 5.64124500 & 8.64078800 \\
\hline $\mathrm{Cl}$ & 0.00000000 & 8.64078800 & 5.64124500 \\
\hline $\mathrm{Cl}$ & 8.64078800 & 0.00000000 & 5.64124500 \\
\hline $\mathrm{Cl}$ & 5.64124500 & 0.00000000 & 8.64078800 \\
\hline $\mathrm{Cl}$ & 8.64078800 & 5.64124500 & 0.00000000 \\
\hline $\mathrm{Cl}$ & 5.64124500 & 8.64078800 & 0.00000000 \\
\hline $\mathrm{Cl}$ & 8.64078800 & 0.00000000 & -5.64124500 \\
\hline $\mathrm{Cl}$ & 5.64124500 & 0.00000000 & -8.64078800 \\
\hline $\mathrm{Cl}$ & 0.00000000 & 5.64124500 & -8.64078800 \\
\hline $\mathrm{Cl}$ & 0.00000000 & 8.64078800 & -5.64124500 \\
\hline
\end{tabular}

Output coordinates:

$\begin{array}{lrrr}\mathrm{Mg} & 1.638455 & -7.395889 & -4.515137 \\ \mathrm{Mg} & -1.638455 & -7.395889 & -4.515137 \\ \mathrm{Mg} & 1.638455 & -4.515137 & -7.395889 \\ \mathrm{Mg} & 4.515137 & -7.395889 & -1.638455 \\ \mathrm{Mg} & -1.638455 & -4.515137 & -7.395889 \\ \mathrm{Mg} & -4.515137 & -7.395889 & -1.638455 \\ \mathrm{Mg} & 4.515137 & -1.638455 & -7.395889 \\ \mathrm{Mg} & 4.515137 & -7.395889 & 1.638455\end{array}$




\begin{tabular}{|c|c|c|c|}
\hline $\mathrm{Mg}$ & 7.395889 & -4.515137 & -1.638455 \\
\hline $\mathrm{Mg}$ & -4.515137 & -1.638455 & -7.395889 \\
\hline $\mathrm{Mg}$ & -4.515137 & -7.395889 & 1.638455 \\
\hline $\mathrm{Mg}$ & -7.395889 & -4.515137 & -1.638455 \\
\hline $\mathrm{Mg}$ & 4.515137 & 1.638455 & -7.395889 \\
\hline $\mathrm{Mg}$ & 7.395889 & -1.638455 & -4.515137 \\
\hline $\mathrm{Mg}$ & 7.395889 & -4.515137 & 1.638455 \\
\hline $\mathrm{Mg}$ & 1.638455 & -7.395889 & 4.515137 \\
\hline $\mathrm{Mg}$ & -4.515137 & 1.638455 & -7.395889 \\
\hline $\mathrm{Mg}$ & -7.395889 & -1.638455 & -4.515137 \\
\hline $\mathrm{Mg}$ & -7.395889 & -4.515137 & 1.638455 \\
\hline $\mathrm{Mg}$ & -1.638455 & -7.395889 & 4.515137 \\
\hline $\mathrm{Mg}$ & 7.395889 & 1.638455 & -4.515137 \\
\hline $\mathrm{Mg}$ & 1.638455 & 4.515137 & -7.395889 \\
\hline $\mathrm{Mg}$ & 7.395889 & -1.638455 & 4.515137 \\
\hline $\mathrm{Mg}$ & 1.638455 & -4.515137 & 7.395889 \\
\hline $\mathrm{Mg}$ & -7.395889 & 1.638455 & -4.515137 \\
\hline $\mathrm{Mg}$ & -1.638455 & 4.515137 & -7.395889 \\
\hline $\mathrm{Mg}$ & -7.395889 & -1.638455 & 4.515137 \\
\hline $\mathrm{Mg}$ & -1.638455 & -4.515137 & 7.395889 \\
\hline $\mathrm{Mg}$ & 7.395889 & 4.515137 & -1.638455 \\
\hline $\mathrm{Mg}$ & 1.638455 & 7.395889 & -4.515137 \\
\hline $\mathrm{Mg}$ & 7.395889 & 1.638455 & 4.515137 \\
\hline $\mathrm{Mg}$ & 4.515137 & -1.638455 & 7.395889 \\
\hline $\mathrm{Mg}$ & -7.395889 & 4.515137 & -1.638455 \\
\hline $\mathrm{Mg}$ & -1.638455 & 7.395889 & -4.515137 \\
\hline $\mathrm{Mg}$ & -7.395889 & 1.638455 & 4.515137 \\
\hline $\mathrm{Mg}$ & -4.515137 & -1.638455 & 7.395889 \\
\hline $\mathrm{Mg}$ & 7.395889 & 4.515137 & 1.638455 \\
\hline $\mathrm{Mg}$ & 4.515137 & 7.395889 & -1.638455 \\
\hline $\mathrm{Mg}$ & 4.515137 & 1.638455 & 7.395889 \\
\hline $\mathrm{Mg}$ & -7.395889 & 4.515137 & 1.638455 \\
\hline $\mathrm{Mg}$ & -4.515137 & 7.395889 & -1.638455 \\
\hline $\mathrm{Mg}$ & -4.515137 & 1.638455 & 7.395889 \\
\hline $\mathrm{Mg}$ & 4.515137 & 7.395889 & 1.638455 \\
\hline $\mathrm{Mg}$ & 1.638455 & 4.515137 & 7.395889 \\
\hline $\mathrm{Mg}$ & -4.515137 & 7.395889 & 1.638455 \\
\hline $\mathrm{Mg}$ & -1.638455 & 4.515137 & 7.395889 \\
\hline $\mathrm{Mg}$ & 1.638455 & 7.395889 & 4.515137 \\
\hline $\mathrm{Mg}$ & -1.638455 & 7.395889 & 4.515137 \\
\hline $\mathrm{Cl}$ & 3.172707 & 3.172707 & 8.591496 \\
\hline $\mathrm{Cl}$ & 3.276756 & 0.000000 & 6.189707 \\
\hline $\mathrm{Cl}$ & 3.172707 & -3.172707 & 8.591496 \\
\hline $\mathrm{Cl}$ & 0.000000 & -3.276756 & 6.189707 \\
\hline $\mathrm{Cl}$ & -3.172707 & -3.172707 & 8.591496 \\
\hline $\mathrm{Cl}$ & -3.276756 & 0.000000 & 6.189707 \\
\hline $\mathrm{Cl}$ & -3.172707 & 3.172707 & 8.591496 \\
\hline $\mathrm{Cl}$ & 0.000000 & 3.276756 & 6.189707 \\
\hline $\mathrm{Cl}$ & 3.172707 & 8.591496 & 3.172707 \\
\hline $\mathrm{Cl}$ & 0.000000 & 6.189707 & 3.276756 \\
\hline $\mathrm{Cl}$ & -3.172707 & 8.591496 & 3.172707 \\
\hline $\mathrm{Cl}$ & -3.276756 & 6.189707 & 0.000000 \\
\hline $\mathrm{Cl}$ & -3.172707 & 8.591496 & -3.172707 \\
\hline $\mathrm{Cl}$ & 0.000000 & 6.189707 & -3.276756 \\
\hline $\mathrm{Cl}$ & 3.172707 & 8.591496 & -3.172707 \\
\hline $\mathrm{Cl}$ & 3.276756 & 6.189707 & 0.000000 \\
\hline $\mathrm{Cl}$ & 6.189707 & 0.000000 & 3.276756 \\
\hline $\mathrm{Cl}$ & 8.591496 & 3.172707 & 3.172707 \\
\hline $\mathrm{Cl}$ & 6.189707 & 3.276756 & 0.000000 \\
\hline $\mathrm{Cl}$ & 8.591496 & 3.172707 & -3.172707 \\
\hline I & 6.189707 & 0.000000 & -3.210 \\
\hline
\end{tabular}




\begin{tabular}{|c|c|c|c|}
\hline $\mathrm{Cl}$ & 8.591496 & -3.172707 & -3.172707 \\
\hline $\mathrm{Cl}$ & 6.189707 & -3.276756 & 0.000000 \\
\hline $\mathrm{Cl}$ & 8.591496 & -3.172707 & 3.172707 \\
\hline $\mathrm{Cl}$ & 3.276756 & -6.189707 & 0.000000 \\
\hline $\mathrm{Cl}$ & 3.172707 & -8.591496 & -3.172707 \\
\hline $\mathrm{Cl}$ & 0.000000 & -6.189707 & -3.276756 \\
\hline $\mathrm{Cl}$ & -3.172707 & -8.591496 & -3.172707 \\
\hline $\mathrm{Cl}$ & -3.276756 & -6.189707 & 0.000000 \\
\hline $\mathrm{Cl}$ & -3.172707 & -8.591496 & 3.172707 \\
\hline $\mathrm{Cl}$ & 0.000000 & -6.189707 & 3.276756 \\
\hline $\mathrm{Cl}$ & 3.172707 & -8.591496 & 3.172707 \\
\hline $\mathrm{Cl}$ & -8.591496 & -3.172707 & 3.172707 \\
\hline $\mathrm{Cl}$ & -6.189707 & -3.276756 & 0.000000 \\
\hline $\mathrm{Cl}$ & -8.591496 & -3.172707 & -3.172707 \\
\hline $\mathrm{Cl}$ & -6.189707 & 0.000000 & -3.276756 \\
\hline $\mathrm{Cl}$ & -8.591496 & 3.172707 & -3.172707 \\
\hline $\mathrm{Cl}$ & -6.189707 & 3.276756 & 0.000000 \\
\hline $\mathrm{Cl}$ & -8.591496 & 3.172707 & 3.172707 \\
\hline $\mathrm{Cl}$ & -6.189707 & 0.000000 & 3.276756 \\
\hline $\mathrm{Cl}$ & -3.276756 & 0.000000 & -6.189707 \\
\hline $\mathrm{Cl}$ & -3.172707 & -3.172707 & -8.591496 \\
\hline $\mathrm{Cl}$ & 0.000000 & -3.276756 & -6.189707 \\
\hline $\mathrm{Cl}$ & 3.172707 & -3.172707 & -8.591496 \\
\hline $\mathrm{Cl}$ & 3.276756 & 0.000000 & -6.189707 \\
\hline $\mathrm{Cl}$ & 3.172707 & 3.172707 & -8.591496 \\
\hline $\mathrm{Cl}$ & 0.000000 & 3.276756 & -6.189707 \\
\hline $\mathrm{Cl}$ & -3.172707 & 3.172707 & -8.591496 \\
\hline $\mathrm{Cl}$ & -6.001542 & 2.849668 & -6.001542 \\
\hline $\mathrm{Cl}$ & -6.001542 & -2.849668 & -6.001542 \\
\hline $\mathrm{Cl}$ & -6.001542 & -6.001542 & -2.849668 \\
\hline $\mathrm{Cl}$ & -6.001542 & -6.001542 & 2.849668 \\
\hline $\mathrm{Cl}$ & -6.001542 & -2.849668 & 6.001542 \\
\hline $\mathrm{Cl}$ & -6.001542 & 2.849668 & 6.001542 \\
\hline $\mathrm{Cl}$ & -6.001542 & 6.001542 & 2.849668 \\
\hline $\mathrm{Cl}$ & -6.001542 & 6.001542 & -2.849668 \\
\hline $\mathrm{Cl}$ & 6.001542 & -2.849668 & 6.001542 \\
\hline $\mathrm{Cl}$ & 6.001542 & -6.001542 & 2.849668 \\
\hline $\mathrm{Cl}$ & 6.001542 & -6.001542 & -2.849668 \\
\hline $\mathrm{Cl}$ & 6.001542 & -2.849668 & -6.001542 \\
\hline $\mathrm{Cl}$ & 6.001542 & 2.849668 & -6.001542 \\
\hline $\mathrm{Cl}$ & 6.001542 & 6.001542 & -2.849668 \\
\hline $\mathrm{Cl}$ & 6.001542 & 6.001542 & 2.849668 \\
\hline $\mathrm{Cl}$ & 6.001542 & 2.849668 & 6.001542 \\
\hline $\mathrm{Cl}$ & 2.849668 & 6.001542 & -6.001542 \\
\hline $\mathrm{Cl}$ & -2.849668 & 6.001542 & -6.001542 \\
\hline $\mathrm{Cl}$ & -2.849668 & -6.001542 & -6.001542 \\
\hline $\mathrm{Cl}$ & 2.849668 & -6.001542 & -6.001542 \\
\hline $\mathrm{Cl}$ & 2.849668 & -6.001542 & 6.001542 \\
\hline $\mathrm{Cl}$ & -2.849668 & -6.001542 & 6.001542 \\
\hline $\mathrm{Cl}$ & -2.849668 & 6.001542 & 6.001542 \\
\hline $\mathrm{Cl}$ & 2.849668 & 6.001542 & 6.001542 \\
\hline $\mathrm{Cl}$ & 5.616168 & -8.729579 & 0.000000 \\
\hline $\mathrm{Cl}$ & 8.729579 & -5.616168 & 0.000000 \\
\hline $\mathrm{Cl}$ & 0.000000 & -8.729579 & -5.616168 \\
\hline $\mathrm{Cl}$ & 0.000000 & -5.616168 & -8.729579 \\
\hline $\mathrm{Cl}$ & -5.616168 & -8.729579 & 0.000000 \\
\hline $\mathrm{Cl}$ & -8.729579 & -5.616168 & 0.000000 \\
\hline $\mathrm{Cl}$ & 0.000000 & -5.616168 & 8.729579 \\
\hline $\mathrm{Cl}$ & 0.000000 & -8.729579 & 5.616168 \\
\hline $\mathrm{Cl}$ & -5.616168 & 0.000000 & -8.729579 \\
\hline $\mathrm{Cl}$ & -8.729579 & 0.000000 & -5.616168 \\
\hline
\end{tabular}




\begin{tabular}{|c|c|c|c|}
\hline $\mathrm{Cl}$ & -5.616168 & 8.729579 & 0.000000 \\
\hline $\mathrm{Cl}$ & -8.729579 & 5.616168 & 0.000000 \\
\hline $\mathrm{Cl}$ & -5.616168 & 0.000000 & 8.729579 \\
\hline $\mathrm{Cl}$ & -8.729579 & 0.000000 & 5.616168 \\
\hline $\mathrm{Cl}$ & 0.000000 & 5.616168 & 8.729579 \\
\hline $\mathrm{Cl}$ & 0.000000 & 8.729579 & 5.616168 \\
\hline $\mathrm{Cl}$ & 8.729579 & 0.000000 & 5.616168 \\
\hline $\mathrm{Cl}$ & 5.616168 & 0.000000 & 8.729579 \\
\hline $\mathrm{Cl}$ & 8.729579 & 5.616168 & 0.000000 \\
\hline $\mathrm{Cl}$ & 5.616168 & 8.729579 & 0.000000 \\
\hline $\mathrm{Cl}$ & 8.729579 & 0.000000 & -5.616168 \\
\hline $\mathrm{Cl}$ & 5.616168 & 0.000000 & -8.729579 \\
\hline $\mathrm{Cl}$ & 0.000000 & 5.616168 & -8.729579 \\
\hline $\mathrm{Cl}$ & 0.000000 & 8.729579 & -5.616168 \\
\hline
\end{tabular}

\section{$\mathrm{Mg}_{60} \mathrm{Cl}_{90}$ (truncated dodecahedron)}

Input keywords:

$\mathrm{HF} /$ gen opt $\operatorname{iop}(2 / 17=5)$

Input coordinates:

$\begin{array}{lccc}\mathrm{Mg} & -1.62937400 & -5.78848300 & -9.36596200 \\ \mathrm{Mg} & -5.00167000 & -3.33836700 & -9.36596200 \\ \mathrm{Mg} & 1.62937400 & -5.78848300 & -9.36596200 \\ \mathrm{Mg} & -5.00167000 & -6.88420800 & -7.17451100 \\ \mathrm{Mg} & -6.00867900 & -0.23911200 & -9.36596200 \\ \mathrm{Mg} & 5.00167000 & -6.88420800 & -7.17451100 \\ \mathrm{Mg} & 5.00167000 & -3.33836700 & -9.36596200 \\ \mathrm{Mg} & -6.00867900 & -8.27023700 & -4.40245400 \\ \mathrm{Mg} & -8.09287200 & 2.62953300 & -7.17451100 \\ \mathrm{Mg} & -4.72057600 & 3.72525900 & -9.36596200 \\ \mathrm{Mg} & 6.00867900 & -8.27023700 & -4.40245400 \\ \mathrm{Mg} & 6.00867900 & -0.23911200 & -9.36596200 \\ \mathrm{Mg} & -4.72057600 & -10.04315700 & -0.85661300 \\ \mathrm{Mg} & -8.09287200 & -7.59304100 & -0.85661300 \\ \mathrm{Mg} & -9.72224600 & 3.15894900 & -4.40245400 \\ \mathrm{Mg} & -2.08419300 & 5.64070300 & -9.36596200 \\ \mathrm{Mg} & 4.72057600 & -10.04315700 & -0.85661300 \\ \mathrm{Mg} & 8.09287200 & -7.59304100 & -0.85661300 \\ \mathrm{Mg} & 8.09287200 & 2.62953300 & -7.17451100 \\ \mathrm{Mg} & 4.72057600 & 3.72525900 & -9.36596200 \\ \mathrm{Mg} & -2.08419300 & -10.89977000 & 0.85661300 \\ \mathrm{Mg} & -9.72224600 & -5.35040000 & 0.85661300 \\ \mathrm{Mg} & -11.01034900 & 1.38602900 & -0.85661300 \\ \mathrm{Mg} & -9.72224600 & 5.35040000 & -0.85661300 \\ \mathrm{Mg} & 0.00000000 & 8.50934900 & -7.17451100 \\ \mathrm{Mg} & 2.08419300 & 5.64070300 & -9.36596200 \\ \mathrm{Mg} & 2.08419300 & -10.89977000 & 0.85661300 \\ \mathrm{Mg} & 9.72224600 & -5.35040000 & 0.85661300 \\ \mathrm{Mg} & 9.72224600 & 3.15894900 & -4.40245400 \\ \mathrm{Mg} & 0.00000000 & -10.22257500 & 4.40245400 \\ \mathrm{Mg} & -9.72224600 & -3.15894900 & 4.40245400 \\ \mathrm{Mg} & -11.01034900 & -1.38602900 & 0.85661300 \\ \mathrm{Mg} & -8.09287200 & 7.59304100 & 0.85661300 \\ \mathrm{Mg} & 0.00000000 & 10.22257500 & -4.40245400 \\ \mathrm{Mg} & 9.72224600 & -3.15894900 & 4.40245400 \\ \mathrm{Mg} & 11.01034900 & -1.38602900 & 0.85661300 \\ \mathrm{Mg} & 11.01034900 & 1.38602900 & -0.85661300 \\ \mathrm{Mg} & 9.72224600 & 5.35040000 & -0.85661300 \\ \mathrm{Mg} & 0.00000000 & -8.50934900 & 7.17451100 \\ \mathrm{Mg} & -8.09287200 & -2.62953300 & 7.17451100\end{array}$




\begin{tabular}{|c|c|c|c|}
\hline $\mathrm{Mg}$ & -6.00867900 & 8.27023700 & 4.40245400 \\
\hline $\mathrm{Mg}$ & -4.72057600 & 10.04315700 & 0.85661300 \\
\hline $\mathrm{Mg}$ & -2.08419300 & 10.89977000 & -0.85661300 \\
\hline $\mathrm{Mg}$ & 2.08419300 & 10.89977000 & -0.85661300 \\
\hline $\mathrm{Mg}$ & 8.09287200 & -2.62953300 & 7.17451100 \\
\hline $\mathrm{Mg}$ & 8.09287200 & 7.59304100 & 0.85661300 \\
\hline $\mathrm{Mg}$ & 2.08419300 & -5.64070300 & 9.36596200 \\
\hline $\mathrm{Mg}$ & -2.08419300 & -5.64070300 & 9.36596200 \\
\hline $\mathrm{Mg}$ & -4.72057600 & -3.72525900 & 9.36596200 \\
\hline $\mathrm{Mg}$ & -6.00867900 & 0.23911200 & 9.36596200 \\
\hline $\mathrm{Mg}$ & -5.00167000 & 6.88420800 & 7.17451100 \\
\hline $\mathrm{Mg}$ & 4.72057600 & 10.04315700 & 0.85661300 \\
\hline $\mathrm{Mg}$ & 4.72057600 & -3.72525900 & 9.36596200 \\
\hline $\mathrm{Mg}$ & 6.00867900 & 0.23911200 & 9.36596200 \\
\hline $\mathrm{Mg}$ & 6.00867900 & 8.27023700 & 4.40245400 \\
\hline $\mathrm{Mg}$ & -5.00167000 & 3.33836700 & 9.36596200 \\
\hline $\mathrm{Mg}$ & -1.62937400 & 5.78848300 & 9.36596200 \\
\hline $\mathrm{Mg}$ & 5.00167000 & 3.33836700 & 9.36596200 \\
\hline $\mathrm{Mg}$ & 5.00167000 & 6.88420800 & 7.17451100 \\
\hline $\mathrm{Mg}$ & 1.62937400 & 5.78848300 & 9.36596200 \\
\hline $\mathrm{Cl}$ & -4.84022200 & 1.57268300 & -10.36629300 \\
\hline $\mathrm{Cl}$ & -6.78768700 & 4.25313900 & -8.31860600 \\
\hline $\mathrm{Cl}$ & -7.99128700 & 0.54884000 & -8.31860600 \\
\hline $\mathrm{Cl}$ & -2.99142200 & -4.11733900 & -10.36629300 \\
\hline $\mathrm{Cl}$ & -6.14248600 & -5.14118200 & -8.31860600 \\
\hline $\mathrm{Cl}$ & -2.99142200 & -7.43056400 & -8.31860600 \\
\hline $\mathrm{Cl}$ & -4.84022200 & -9.97521900 & -3.22929600 \\
\hline $\mathrm{Cl}$ & -7.99128700 & -7.68583700 & -3.22929600 \\
\hline $\mathrm{Cl}$ & -6.78768700 & -9.34244900 & 0.08392900 \\
\hline $\mathrm{Cl}$ & -9.77910800 & -5.22511100 & 3.22929600 \\
\hline $\mathrm{Cl}$ & -10.98270800 & -3.56849800 & -0.08392900 \\
\hline $\mathrm{Cl}$ & -10.98270800 & -1.52081200 & 3.22929600 \\
\hline $\mathrm{Cl}$ & -10.98270800 & 1.52081200 & -3.22929600 \\
\hline $\mathrm{Cl}$ & -9.77910800 & 5.22511100 & -3.22929600 \\
\hline $\mathrm{Cl}$ & -10.98270800 & 3.56849800 & 0.08392900 \\
\hline $\mathrm{Cl}$ & -1.94746500 & 7.76976600 & -8.31860600 \\
\hline $\mathrm{Cl}$ & 0.00000000 & 5.08931000 & -10.36629300 \\
\hline $\mathrm{Cl}$ & 1.94746500 & 7.76976600 & -8.31860600 \\
\hline $\mathrm{Cl}$ & 2.99142200 & -4.11733900 & -10.36629300 \\
\hline $\mathrm{Cl}$ & 2.99142200 & -7.43056400 & -8.31860600 \\
\hline $\mathrm{Cl}$ & 6.14248600 & -5.14118200 & -8.31860600 \\
\hline $\mathrm{Cl}$ & 0.00000000 & -11.54790300 & -0.08392900 \\
\hline $\mathrm{Cl}$ & -1.94746500 & -10.91513300 & 3.22929600 \\
\hline $\mathrm{Cl}$ & 1.94746500 & -10.91513300 & 3.22929600 \\
\hline $\mathrm{Cl}$ & -6.78768700 & -4.25313900 & 8.31860600 \\
\hline $\mathrm{Cl}$ & -7.99128700 & -0.54884000 & 8.31860600 \\
\hline $\mathrm{Cl}$ & -4.84022200 & -1.57268300 & 10.36629300 \\
\hline $\mathrm{Cl}$ & -1.94746500 & -7.76976600 & 8.31860600 \\
\hline $\mathrm{Cl}$ & 0.00000000 & -5.08931000 & 10.36629300 \\
\hline $\mathrm{Cl}$ & 1.94746500 & -7.76976600 & 8.31860600 \\
\hline $\mathrm{Cl}$ & 6.78768700 & -4.25313900 & 8.31860600 \\
\hline $\mathrm{Cl}$ & 7.99128700 & -0.54884000 & 8.31860600 \\
\hline $\mathrm{Cl}$ & 4.84022200 & -1.57268300 & 10.36629300 \\
\hline $\mathrm{Cl}$ & -2.99142200 & 4.11733900 & 10.36629300 \\
\hline $\mathrm{Cl}$ & -6.14248600 & 5.14118200 & 8.31860600 \\
\hline $\mathrm{Cl}$ & -2.99142200 & 7.43056400 & 8.31860600 \\
\hline $\mathrm{Cl}$ & 2.99142200 & 4.11733900 & 10.36629300 \\
\hline $\mathrm{Cl}$ & 2.99142200 & 7.43056400 & 8.31860600 \\
\hline $\mathrm{Cl}$ & 6.14248600 & 5.14118200 & 8.31860600 \\
\hline $\mathrm{Cl}$ & 7.99128700 & 7.68583700 & 3.22929600 \\
\hline $\mathrm{Cl}$ & 4.84022200 & 9.97521900 & 3.22929600 \\
\hline
\end{tabular}




\begin{tabular}{|c|c|c|c|}
\hline $\mathrm{Cl}$ & 6.78768700 & 9.34244900 & -0.08392900 \\
\hline $\mathrm{Cl}$ & 10.98270800 & 3.56849800 & 0.08392900 \\
\hline $\mathrm{Cl}$ & 10.98270800 & 1.52081200 & -3.22929600 \\
\hline $\mathrm{Cl}$ & 9.77910800 & 5.22511100 & -3.22929600 \\
\hline $\mathrm{Cl}$ & 7.99128700 & 0.54884000 & -8.31860600 \\
\hline $\mathrm{Cl}$ & 6.78768700 & 4.25313900 & -8.31860600 \\
\hline $\mathrm{Cl}$ & 4.84022200 & 1.57268300 & -10.36629300 \\
\hline $\mathrm{Cl}$ & 10.98270800 & -1.52081200 & 3.22929600 \\
\hline $\mathrm{Cl}$ & 10.98270800 & -3.56849800 & -0.08392900 \\
\hline $\mathrm{Cl}$ & 9.77910800 & -5.22511100 & 3.22929600 \\
\hline $\mathrm{Cl}$ & 4.84022200 & -9.97521900 & -3.22929600 \\
\hline $\mathrm{Cl}$ & 6.78768700 & -9.34244900 & 0.08392900 \\
\hline $\mathrm{Cl}$ & 7.99128700 & -7.68583700 & -3.22929600 \\
\hline $\mathrm{Cl}$ & -4.84022200 & 9.97521900 & 3.22929600 \\
\hline $\mathrm{Cl}$ & -7.99128700 & 7.68583700 & 3.22929600 \\
\hline $\mathrm{Cl}$ & -6.78768700 & 9.34244900 & -0.08392900 \\
\hline $\mathrm{Cl}$ & -1.94746500 & 10.91513300 & -3.22929600 \\
\hline $\mathrm{Cl}$ & 1.94746500 & 10.91513300 & -3.22929600 \\
\hline $\mathrm{Cl}$ & 0.00000000 & 11.54790300 & 0.08392900 \\
\hline $\mathrm{Cl}$ & -12.75277700 & 0.00000000 & 0.00000000 \\
\hline $\mathrm{Cl}$ & -10.31721300 & 7.49589400 & 0.00000000 \\
\hline $\mathrm{Cl}$ & -6.37638800 & 8.77634600 & 6.70453200 \\
\hline $\mathrm{Cl}$ & -6.37638800 & 2.07181400 & 10.84816000 \\
\hline $\mathrm{Cl}$ & -10.31721300 & -3.35226600 & 6.70453200 \\
\hline $\mathrm{Cl}$ & 0.00000000 & 6.70453200 & 10.84816000 \\
\hline $\mathrm{Cl}$ & -3.94082500 & -5.42408000 & 10.84816000 \\
\hline $\mathrm{Cl}$ & -10.31721300 & -7.49589400 & 0.00000000 \\
\hline $\mathrm{Cl}$ & -10.31721300 & 3.35226600 & -6.70453200 \\
\hline $\mathrm{Cl}$ & -3.94082500 & 12.12861200 & 0.00000000 \\
\hline $\mathrm{Cl}$ & -6.37638800 & -8.77634600 & -6.70453200 \\
\hline $\mathrm{Cl}$ & -3.94082500 & -12.12861200 & 0.00000000 \\
\hline $\mathrm{Cl}$ & 3.94082500 & -12.12861200 & 0.00000000 \\
\hline $\mathrm{Cl}$ & 6.37638800 & -8.77634600 & -6.70453200 \\
\hline $\mathrm{Cl}$ & 0.00000000 & -6.70453200 & -10.84816000 \\
\hline $\mathrm{Cl}$ & 10.31721300 & -7.49589400 & 0.00000000 \\
\hline $\mathrm{Cl}$ & 0.00000000 & -10.84816000 & 6.70453200 \\
\hline $\mathrm{Cl}$ & -6.37638800 & -2.07181400 & -10.84816000 \\
\hline $\mathrm{Cl}$ & 6.37638800 & -2.07181400 & -10.84816000 \\
\hline $\mathrm{Cl}$ & 3.94082500 & -5.42408000 & 10.84816000 \\
\hline $\mathrm{Cl}$ & 6.37638800 & 2.07181400 & 10.84816000 \\
\hline $\mathrm{Cl}$ & 6.37638800 & 8.77634600 & 6.70453200 \\
\hline $\mathrm{Cl}$ & 10.31721300 & 7.49589400 & 0.00000000 \\
\hline $\mathrm{Cl}$ & 12.75277700 & 0.00000000 & 0.00000000 \\
\hline $\mathrm{Cl}$ & 10.31721300 & -3.35226600 & 6.70453200 \\
\hline $\mathrm{Cl}$ & 10.31721300 & 3.35226600 & -6.70453200 \\
\hline $\mathrm{Cl}$ & 3.94082500 & 5.42408000 & -10.84816000 \\
\hline $\mathrm{Cl}$ & 0.00000000 & 10.84816000 & -6.70453200 \\
\hline $\mathrm{Cl}$ & 3.94082500 & 12.12861200 & 0.00000000 \\
\hline $\mathrm{Cl}$ & -3.94082500 & 5.42408000 & -10.84816000 \\
\hline $\mathrm{Cl}$ & 4.63021900 & 1.50444900 & 7.87739900 \\
\hline $\mathrm{Cl}$ & 2.86163300 & -3.93869900 & 7.87739900 \\
\hline $\mathrm{Cl}$ & -2.86163300 & -3.93869900 & 7.87739900 \\
\hline $\mathrm{Cl}$ & -4.63021900 & 1.50444900 & 7.87739900 \\
\hline $\mathrm{Cl}$ & 0.00000000 & 4.86850000 & 7.87739900 \\
\hline $\mathrm{Cl}$ & 7.49185200 & -2.43425000 & 4.86850000 \\
\hline $\mathrm{Cl}$ & 7.49185200 & -5.44314900 & 0.00000000 \\
\hline $\mathrm{Cl}$ & 2.86163300 & -8.80720000 & 0.00000000 \\
\hline $\mathrm{Cl}$ & 0.00000000 & -7.87739900 & 4.86850000 \\
\hline $\mathrm{Cl}$ & 4.63021900 & -6.37295000 & -4.86850000 \\
\hline $\mathrm{Cl}$ & 0.00000000 & -4.86850000 & -7.87739900 \\
\hline $\mathrm{Cl}$ & -4.63021900 & -6.37295000 & -4.86850000 \\
\hline
\end{tabular}




$\begin{array}{lrrr}\mathrm{Cl} & -2.86163300 & -8.80720000 & 0.00000000 \\ \mathrm{Cl} & -7.49185200 & -5.44314900 & 0.00000000 \\ \mathrm{Cl} & -7.49185200 & -2.43425000 & 4.86850000 \\ \mathrm{Cl} & -9.26043800 & 0.00000000 & 0.00000000 \\ \mathrm{Cl} & -7.49185200 & 5.44314900 & 0.00000000 \\ \mathrm{Cl} & -4.63021900 & 6.37295000 & 4.86850000 \\ \mathrm{Cl} & -2.86163300 & 8.80720000 & 0.00000000 \\ \mathrm{Cl} & 2.86163300 & 8.80720000 & 0.00000000 \\ \mathrm{Cl} & 4.63021900 & 6.37295000 & 4.86850000 \\ \mathrm{Cl} & 7.49185200 & 5.44314900 & 0.00000000 \\ \mathrm{Cl} & 9.26043800 & 0.00000000 & 0.00000000 \\ \mathrm{Cl} & 7.49185200 & 2.43425000 & -4.86850000 \\ \mathrm{Cl} & 4.63021900 & -1.50444900 & -7.87739900 \\ \mathrm{Cl} & 2.86163300 & 3.93869900 & -7.87739900 \\ \mathrm{Cl} & -2.86163300 & 3.93869900 & -7.87739900 \\ \mathrm{Cl} & -4.63021900 & -1.50444900 & -7.87739900 \\ \mathrm{Cl} & -7.49185200 & 2.43425000 & -4.86850000 \\ \mathrm{Cl} & 0.00000000 & 7.87739900 & -4.86850000\end{array}$

Output coordinates:

$\begin{array}{lccc}\mathrm{Mg} & -1.635343 & -5.771343 & -9.338230 \\ \mathrm{Mg} & -4.983525 & -3.338747 & -9.338230 \\ \mathrm{Mg} & 1.635343 & -5.771343 & -9.338230 \\ \mathrm{Mg} & -4.983525 & -6.859234 & -7.162449 \\ \mathrm{Mg} & -5.994222 & -0.228140 & -9.338230 \\ \mathrm{Mg} & 4.983525 & -6.859233 & -7.162449 \\ \mathrm{Mg} & 4.983525 & -3.338746 & -9.338230 \\ \mathrm{Mg} & -5.994222 & -8.250339 & -4.380238 \\ \mathrm{Mg} & -8.063513 & 2.619994 & -7.162449 \\ \mathrm{Mg} & -4.715331 & 3.707884 & -9.338230 \\ \mathrm{Mg} & 5.994223 & -8.250339 & -4.380238 \\ \mathrm{Mg} & 5.994222 & -0.228139 & -9.338230 \\ \mathrm{Mg} & -4.715330 & -10.010583 & -0.859751 \\ \mathrm{Mg} & -8.063512 & -7.577986 & -0.859751 \\ \mathrm{Mg} & -9.698856 & 3.151349 & -4.380238 \\ \mathrm{Mg} & -2.069291 & 5.630345 & -9.338230 \\ \mathrm{Mg} & 4.715331 & -10.010582 & -0.859751 \\ \mathrm{Mg} & 8.063513 & -7.577986 & -0.859751 \\ \mathrm{Mg} & 8.063512 & 2.619994 & -7.162449 \\ \mathrm{Mg} & 4.715330 & 3.707885 & -9.338230 \\ \mathrm{Mg} & -2.069290 & -10.870333 & 0.859751 \\ \mathrm{Mg} & -9.698855 & -5.327130 & 0.859751 \\ \mathrm{Mg} & -10.977747 & 1.391105 & -0.859751 \\ \mathrm{Mg} & -9.698856 & 5.327129 & -0.859751 \\ \mathrm{Mg} & -0.000000 & 8.478479 & -7.162449 \\ \mathrm{Mg} & 2.069290 & 5.630345 & -9.338230 \\ \mathrm{Mg} & 2.069291 & -10.870333 & 0.859751 \\ \mathrm{Mg} & 9.698856 & -5.327129 & 0.859751 \\ \mathrm{Mg} & 9.698855 & 3.151350 & -4.380238 \\ \mathrm{Mg} & 0.000000 & -10.197980 & 4.380238 \\ \mathrm{Mg} & -9.698855 & -3.151350 & 4.380238 \\ \mathrm{Mg} & -10.977747 & -1.391106 & 0.859751 \\ \mathrm{Mg} & -8.063513 & 7.577986 & 0.859751 \\ \mathrm{Mg} & -0.000000 & 10.197980 & -4.380238 \\ \mathrm{Mg} & 9.698856 & -3.151349 & 4.380238 \\ \mathrm{Mg} & 10.977747 & -1.391105 & 0.859751 \\ \mathrm{Mg} & 10.977747 & 1.391106 & -0.859751 \\ \mathrm{Mg} & 9.698855 & 5.327130 & -0.859751 \\ \mathrm{Mg} & 0.000000 & -8.478479 & 7.162449 \\ \mathrm{Mg} & -8.063512 & -2.619994 & 7.162449 \\ \mathrm{Mg} & -5.994223 & 8.250339 & 4.380238 \\ \mathrm{Mg} & -4.715331 & 10.010582 & 0.859751\end{array}$




\begin{tabular}{|c|c|c|c|}
\hline $\mathrm{Mg}$ & -2.069291 & 10.870333 & $3-0.859751$ \\
\hline $\mathrm{Mg}$ & 2.069290 & 10.870333 & -0.859751 \\
\hline $\mathrm{Mg}$ & 8.063513 & -2.619994 & 7.162449 \\
\hline $\mathrm{Mg}$ & 8.063512 & 7.577986 & 0.859751 \\
\hline $\mathrm{Mg}$ & 2.069291 & -5.630345 & 9.338230 \\
\hline $\mathrm{Mg}$ & -2.069290 & -5.630345 & 9.338230 \\
\hline $\mathrm{Mg}$ & -4.715330 & -3.707885 & 9.338230 \\
\hline $\mathrm{Mg}$ & -5.994222 & 0.228139 & 9.338230 \\
\hline $\mathrm{Mg}$ & -4.983525 & 6.859233 & 7.162449 \\
\hline $\mathrm{Mg}$ & 4.715330 & 10.010583 & 0.859751 \\
\hline $\mathrm{Mg}$ & 4.715331 & -3.707884 & 9.338230 \\
\hline $\mathrm{Mg}$ & 5.994222 & 0.228140 & 9.338230 \\
\hline $\mathrm{Mg}$ & 5.994222 & 8.250339 & 4.380238 \\
\hline $\mathrm{Mg}$ & -4.983525 & 3.338746 & 9.338230 \\
\hline $\mathrm{Mg}$ & -1.635343 & 5.771343 & 9.338230 \\
\hline $\mathrm{Mg}$ & 4.983525 & 3.338747 & 9.338230 \\
\hline $\mathrm{Mg}$ & 4.983525 & 6.859234 & 7.162449 \\
\hline $\mathrm{Mg}$ & 1.635343 & 5.771343 & 9.338230 \\
\hline $\mathrm{Cl}$ & -4.840172 & 1.572667 & -10.346548 \\
\hline $\mathrm{Cl}$ & -6.777293 & 4.238885 & -8.309739 \\
\hline $\mathrm{Cl}$ & -7.974500 & 0.554262 & -8.309739 \\
\hline $\mathrm{Cl}$ & -2.991391 & -4.117297 & -10.346548 \\
\hline $\mathrm{Cl}$ & -6.125718 & -5.135701 & -8.309739 \\
\hline $\mathrm{Cl}$ & -2.991391 & -7.412923 & -8.309739 \\
\hline $\mathrm{Cl}$ & -4.840172 & -9.957553 & -3.220481 \\
\hline $\mathrm{Cl}$ & -7.974499 & -7.680331 & -3.220481 \\
\hline $\mathrm{Cl}$ & -6.777293 & -9.328144 & 0.075146 \\
\hline $\mathrm{Cl}$ & -9.768684 & -5.210848 & 3.220481 \\
\hline $\mathrm{Cl}$ & -10.965890 & -3.563034 & -0.075146 \\
\hline $\mathrm{Cl}$ & -10.965890 & -1.526225 & 3.220481 \\
\hline $\mathrm{Cl}$ & -10.965891 & 1.526224 & -3.220481 \\
\hline $\mathrm{Cl}$ & -9.768684 & 5.210847 & -3.220481 \\
\hline $\mathrm{Cl}$ & -10.965891 & 3.563033 & 0.075146 \\
\hline $\mathrm{Cl}$ & -1.937121 & 7.755476 & -8.309739 \\
\hline $\mathrm{Cl}$ & -0.000000 & 5.089258 & -10.346548 \\
\hline $\mathrm{Cl}$ & 1.937120 & 7.755476 & -8.309739 \\
\hline $\mathrm{Cl}$ & 2.991391 & -4.117296 & -10.346548 \\
\hline $\mathrm{Cl}$ & 2.991391 & -7.412923 & -8.309739 \\
\hline $\mathrm{Cl}$ & 6.125719 & -5.135701 & -8.309739 \\
\hline $\mathrm{Cl}$ & 0.000001 & -11.530220 & -0.075146 \\
\hline $\mathrm{Cl}$ & -1.937120 & -10.900811 & 3.220481 \\
\hline $\mathrm{Cl}$ & 1.937121 & -10.900811 & 3.220481 \\
\hline $\mathrm{Cl}$ & -6.777293 & -4.238886 & 8.309739 \\
\hline $\mathrm{Cl}$ & -7.974500 & -0.554263 & 8.309739 \\
\hline $\mathrm{Cl}$ & -4.840172 & -1.572668 & 10.346548 \\
\hline $\mathrm{Cl}$ & -1.937120 & -7.755476 & 8.309739 \\
\hline $\mathrm{Cl}$ & 0.000000 & -5.089258 & 10.346548 \\
\hline $\mathrm{Cl}$ & 1.937121 & -7.755476 & 8.309739 \\
\hline $\mathrm{Cl}$ & 6.777293 & -4.238885 & 8.309739 \\
\hline $\mathrm{Cl}$ & 7.974500 & -0.554262 & 8.309739 \\
\hline $\mathrm{Cl}$ & 4.840172 & -1.572667 & 10.346548 \\
\hline $\mathrm{Cl}$ & -2.991391 & 4.117296 & 10.346548 \\
\hline $\mathrm{Cl}$ & -6.125719 & 5.135701 & 8.309739 \\
\hline $\mathrm{Cl}$ & -2.991391 & 7.412923 & 8.309739 \\
\hline $\mathrm{Cl}$ & 2.991391 & 4.117297 & 10.346548 \\
\hline $\mathrm{Cl}$ & 2.991391 & 7.412923 & 8.309739 \\
\hline $\mathrm{Cl}$ & 6.125718 & 5.135701 & 8.309739 \\
\hline $\mathrm{Cl}$ & 7.974499 & 7.680331 & 3.220481 \\
\hline $\mathrm{Cl}$ & 4.840172 & 9.957553 & 3.220481 \\
\hline $\mathrm{Cl}$ & 6.777293 & 9.328144 & -0.075146 \\
\hline & 10.965890 & 3.563034 & 0.07514 \\
\hline
\end{tabular}




\begin{tabular}{|c|c|c|c|}
\hline $\mathrm{Cl}$ & 10.965890 & 1.526225 & -3.220481 \\
\hline $\mathrm{Cl}$ & 9.768684 & 5.210848 & -3.220481 \\
\hline $\mathrm{Cl}$ & 7.974500 & 0.554263 & -8.309739 \\
\hline $\mathrm{Cl}$ & 6.777293 & 4.238886 & -8.309739 \\
\hline $\mathrm{Cl}$ & 4.840172 & 1.572668 & -10.346548 \\
\hline $\mathrm{Cl}$ & 10.965891 & -1.526224 & 3.220481 \\
\hline $\mathrm{Cl}$ & 10.965891 & -3.563033 & -0.075146 \\
\hline $\mathrm{Cl}$ & 9.768684 & -5.210847 & 3.220481 \\
\hline $\mathrm{Cl}$ & 4.840173 & -9.957552 & -3.220481 \\
\hline $\mathrm{Cl}$ & 6.777293 & -9.328143 & 0.075146 \\
\hline $\mathrm{Cl}$ & 7.974500 & -7.680330 & -3.220481 \\
\hline $\mathrm{Cl}$ & -4.840173 & 9.957552 & 3.220481 \\
\hline $\mathrm{Cl}$ & -7.974500 & 7.680330 & 3.220481 \\
\hline $\mathrm{Cl}$ & -6.777293 & 9.328143 & -0.075146 \\
\hline $\mathrm{Cl}$ & -1.937121 & 10.900811 & -3.220481 \\
\hline $\mathrm{Cl}$ & 1.937120 & 10.900811 & -3.220481 \\
\hline $\mathrm{Cl}$ & -0.000001 & 11.530220 & 0.075146 \\
\hline $\mathrm{Cl}$ & -12.706556 & -0.000001 & 0.000000 \\
\hline $\mathrm{Cl}$ & -10.279820 & 7.468725 & 0.000000 \\
\hline $\mathrm{Cl}$ & -6.353278 & 8.744536 & 6.680232 \\
\hline $\mathrm{Cl}$ & -6.353278 & 2.064305 & 10.808842 \\
\hline $\mathrm{Cl}$ & -10.279819 & -3.340116 & 6.680232 \\
\hline $\mathrm{Cl}$ & -0.000000 & 6.680232 & 10.808842 \\
\hline $\mathrm{Cl}$ & -3.926541 & -5.404421 & 10.808842 \\
\hline $\mathrm{Cl}$ & -10.279819 & -7.468726 & 0.000000 \\
\hline $\mathrm{Cl}$ & -10.279820 & 3.340115 & -6.680232 \\
\hline $\mathrm{Cl}$ & -3.926542 & 12.084652 & 0.000000 \\
\hline $\mathrm{Cl}$ & -6.353277 & -8.744537 & -6.680232 \\
\hline $\mathrm{Cl}$ & -3.926541 & -12.084653 & 0.000000 \\
\hline $\mathrm{Cl}$ & 3.926542 & -12.084652 & 0.000000 \\
\hline $\mathrm{Cl}$ & 6.353278 & -8.744536 & -6.680232 \\
\hline $\mathrm{Cl}$ & 0.000000 & -6.680232 & -10.808842 \\
\hline $\mathrm{Cl}$ & 10.279820 & -7.468725 & 0.000000 \\
\hline $\mathrm{Cl}$ & 0.000001 & -10.808842 & 6.680232 \\
\hline $\mathrm{Cl}$ & -6.353278 & -2.064305 & -10.808842 \\
\hline $\mathrm{Cl}$ & 6.353278 & -2.064305 & -10.808842 \\
\hline $\mathrm{Cl}$ & 3.926542 & -5.404421 & 10.808842 \\
\hline $\mathrm{Cl}$ & 6.353278 & 2.064305 & 10.808842 \\
\hline $\mathrm{Cl}$ & 6.353277 & 8.744537 & 6.680232 \\
\hline $\mathrm{Cl}$ & 10.279819 & 7.468726 & 0.000000 \\
\hline $\mathrm{Cl}$ & 12.706556 & 0.000001 & 0.000000 \\
\hline $\mathrm{Cl}$ & 10.279820 & -3.340115 & 6.680232 \\
\hline $\mathrm{Cl}$ & 10.279819 & 3.340116 & -6.680232 \\
\hline $\mathrm{Cl}$ & 3.926541 & 5.404421 & -10.808842 \\
\hline $\mathrm{Cl}$ & -0.000001 & 10.808842 & -6.680232 \\
\hline $\mathrm{Cl}$ & 3.926541 & 12.084653 & 0.000000 \\
\hline $\mathrm{Cl}$ & -3.926542 & 5.404421 & -10.808842 \\
\hline $\mathrm{Cl}$ & 4.622778 & 1.502032 & 7.864740 \\
\hline $\mathrm{Cl}$ & 2.857034 & -3.932370 & 7.864740 \\
\hline $\mathrm{Cl}$ & -2.857034 & -3.932370 & 7.864740 \\
\hline $\mathrm{Cl}$ & -4.622778 & 1.502031 & 7.864740 \\
\hline $\mathrm{Cl}$ & -0.000000 & 4.860676 & 7.864740 \\
\hline $\mathrm{Cl}$ & 7.479812 & -2.430338 & 4.860676 \\
\hline $\mathrm{Cl}$ & 7.479812 & -5.434401 & 0.000000 \\
\hline $\mathrm{Cl}$ & 2.857034 & -8.793046 & 0.000000 \\
\hline $\mathrm{Cl}$ & 0.000000 & -7.864740 & 4.860676 \\
\hline $\mathrm{Cl}$ & 4.622778 & -6.362708 & -4.860676 \\
\hline $\mathrm{Cl}$ & 0.000000 & -4.860676 & -7.864740 \\
\hline $\mathrm{Cl}$ & -4.622778 & -6.362708 & -4.860676 \\
\hline $\mathrm{Cl}$ & -2.857033 & -8.793046 & 0.000000 \\
\hline $\mathrm{Cl}$ & -7479812 & -5.434402 & 0.00000 \\
\hline
\end{tabular}




$\begin{array}{lrrr}\mathrm{Cl} & -7.479812 & -2.430339 & 4.860676 \\ \mathrm{Cl} & -9.245556 & -0.000000 & 0.000000 \\ \mathrm{Cl} & -7.479812 & 5.434401 & 0.000000 \\ \mathrm{Cl} & -4.622778 & 6.362708 & 4.860676 \\ \mathrm{Cl} & -2.857034 & 8.793046 & 0.000000 \\ \mathrm{Cl} & 2.857033 & 8.793046 & 0.000000 \\ \mathrm{Cl} & 4.622778 & 6.362708 & 4.860676 \\ \mathrm{Cl} & 7.479812 & 5.434402 & 0.000000 \\ \mathrm{Cl} & 9.245556 & 0.000000 & 0.000000 \\ \mathrm{Cl} & 7.479812 & 2.430339 & -4.860676 \\ \mathrm{Cl} & 4.622778 & -1.502031 & -7.864740 \\ \mathrm{Cl} & 2.857034 & 3.932370 & -7.864740 \\ \mathrm{Cl} & -2.857034 & 3.932370 & -7.864740 \\ \mathrm{Cl} & -4.622778 & -1.502032 & -7.864740 \\ \mathrm{Cl} & -7.479812 & 2.430338 & -4.860676 \\ \mathrm{Cl} & -0.000000 & 7.864740 & -4.860676\end{array}$

\section{$\mathrm{Mg}_{60} \mathrm{Cl}_{90}$ (truncated icosahedron)}

Input keywords:

$\begin{array}{lccc}\text { HF/gen opt iop }(2 / 17=5) & & \\ \text { Input coordinates: } & & \\ \mathrm{Mg} & 7.65273100 & -6.63409500 & 1.40596300 \\ \mathrm{Mg} & 3.94457500 & -9.32822800 & 1.40596300 \\ \mathrm{Mg} & 2.29176700 & -9.86525800 & -1.40596300 \\ \mathrm{Mg} & 3.70815700 & -7.91576500 & -5.30494900 \\ \mathrm{Mg} & 6.38245600 & -5.97277200 & -5.30494900 \\ \mathrm{Mg} & 8.67422300 & -5.22813200 & -1.40596300 \\ \mathrm{Mg} & 7.65273100 & -4.22438800 & 5.30494900 \\ \mathrm{Mg} & 3.94457500 & -5.42924200 & 7.71465500 \\ \mathrm{Mg} & 1.65280800 & -8.58358800 & 5.30494900 \\ \mathrm{Mg} & -1.65280800 & -8.58358800 & 5.30494900 \\ \mathrm{Mg} & -2.29176700 & -9.86525800 & -1.40596300 \\ \mathrm{Mg} & 0.00000000 & -6.71091200 & -7.71465500 \\ \mathrm{Mg} & 0.00000000 & -3.89898600 & -9.45252100 \\ \mathrm{Mg} & 3.70815700 & -1.20485300 & -9.45252100 \\ \mathrm{Mg} & 6.38245600 & -2.07378600 & -7.71465500 \\ \mathrm{Mg} & 8.67422300 & 1.08056100 & -5.30494900 \\ \mathrm{Mg} & 10.09061300 & -0.86893300 & -1.40596300 \\ \mathrm{Mg} & 10.09061300 & 0.86893300 & 1.40596300 \\ \mathrm{Mg} & 8.67422300 & -1.08056100 & 5.30494900 \\ \mathrm{Mg} & 2.29176700 & -3.15434600 & 9.45252100 \\ \mathrm{Mg} & 3.70815700 & 1.20485300 & 9.45252100 \\ \mathrm{Mg} & 6.38245600 & 2.07378600 & 7.71465500 \\ \mathrm{Mg} & 6.38245600 & 5.97277200 & 5.30494900 \\ \mathrm{Mg} & 8.67422300 & 5.22813200 & 1.40596300 \\ \mathrm{Mg} & 7.65273100 & 6.63409500 & -1.40596300 \\ \mathrm{Mg} & 7.65273100 & 4.22438800 & -5.30494900 \\ \mathrm{Mg} & 3.94457500 & 5.42924200 & -7.71465500 \\ \mathrm{Mg} & 2.29176700 & 3.15434600 & -9.45252100 \\ \mathrm{Mg} & -3.70815700 & -7.91576500 & -5.30494900 \\ \mathrm{Mg} & -7.65273100 & 4.22438800 & -5.30494900 \\ \mathrm{Mg} & -8.67422300 & 1.08056100 & -5.30494900 \\ \mathrm{Mg} & -10.09061300 & -0.86893300 & -1.40596300 \\ \mathrm{Mg} & -10.09061300 & 0.86893300 & 1.40596300 \\ \mathrm{Mg} & -8.67422300 & 5.22813200 & 1.40596300 \\ \mathrm{Mg} & -3.94457500 & 9.32822800 & -1.40596300 \\ \mathrm{Mg} & -1.65280800 & 8.58358800 & -5.30494900 \\ \mathrm{Mg} & -3.94457500 & 5.42924200 & -7.71465500 \\ \mathrm{Mg} & -2.29176700 & 3.15434600 & -9.45252100\end{array}$




\begin{tabular}{|c|c|c|c|}
\hline $\mathrm{Mg}$ & -3.70815700 & -1.20485300 & -9.45252100 \\
\hline $\mathrm{Mg}$ & -6.38245600 & -2.07378600 & -7.71465500 \\
\hline $\mathrm{Mg}$ & -8.67422300 & -5.22813200 & -1.40596300 \\
\hline $\mathrm{Mg}$ & -7.65273100 & -6.63409500 & 1.40596300 \\
\hline $\mathrm{Mg}$ & -7.65273100 & -4.22438800 & 5.30494900 \\
\hline $\mathrm{Mg}$ & -8.67422300 & -1.08056100 & 5.30494900 \\
\hline $\mathrm{Mg}$ & -6.38245600 & 2.07378600 & 7.71465500 \\
\hline $\mathrm{Mg}$ & -6.38245600 & 5.97277200 & 5.30494900 \\
\hline $\mathrm{Mg}$ & -3.70815700 & 7.91576500 & 5.30494900 \\
\hline $\mathrm{Mg}$ & -2.29176700 & 9.86525800 & 1.40596300 \\
\hline $\mathrm{Mg}$ & 1.65280800 & 8.58358800 & -5.30494900 \\
\hline $\mathrm{Mg}$ & 3.94457500 & 9.32822800 & -1.40596300 \\
\hline $\mathrm{Mg}$ & 2.29176700 & 9.86525800 & 1.40596300 \\
\hline $\mathrm{Mg}$ & 3.70815700 & 7.91576500 & 5.30494900 \\
\hline $\mathrm{Mg}$ & 0.00000000 & 6.71091200 & 7.71465500 \\
\hline $\mathrm{Mg}$ & 0.00000000 & 3.89898600 & 9.45252100 \\
\hline $\mathrm{Mg}$ & -3.70815700 & 1.20485300 & 9.45252100 \\
\hline $\mathrm{Mg}$ & -2.29176700 & -3.15434600 & 9.45252100 \\
\hline $\mathrm{Mg}$ & -3.94457500 & -5.42924200 & 7.71465500 \\
\hline $\mathrm{Mg}$ & -6.38245600 & -5.97277200 & -5.30494900 \\
\hline $\mathrm{Mg}$ & -3.94457500 & -9.32822800 & 1.40596300 \\
\hline $\mathrm{Mg}$ & -7.65273100 & 6.63409500 & -1.40596300 \\
\hline $\mathrm{Cl}$ & -6.13827200 & 4.88669700 & -7.00248200 \\
\hline $\mathrm{Cl}$ & -8.23191100 & 5.56696100 & -3.44057100 \\
\hline $\mathrm{Cl}$ & -7.83829500 & 6.10872700 & 3.44057100 \\
\hline $\mathrm{Cl}$ & -9.93193300 & 3.22708100 & 1.23919000 \\
\hline $\mathrm{Cl}$ & -9.93193300 & -3.22708100 & -1.23919000 \\
\hline $\mathrm{Cl}$ & -9.93193300 & 0.33483000 & -3.44057100 \\
\hline $\mathrm{Cl}$ & -2.75069400 & 7.34791600 & -7.00248200 \\
\hline $\mathrm{Cl}$ & -2.75069400 & 9.54929800 & -3.44057100 \\
\hline $\mathrm{Cl}$ & -6.13827200 & 8.44860700 & -1.23919000 \\
\hline $\mathrm{Cl}$ & 0.00000000 & 10.44305300 & 1.23919000 \\
\hline $\mathrm{Cl}$ & -3.38757800 & 9.34236200 & 3.44057100 \\
\hline $\mathrm{Cl}$ & -6.54435500 & 4.32777200 & 7.00248200 \\
\hline $\mathrm{Cl}$ & -7.83829500 & 0.34543500 & 7.00248200 \\
\hline $\mathrm{Cl}$ & -9.93193300 & -0.33483000 & 3.44057100 \\
\hline $\mathrm{Cl}$ & -6.13827200 & -4.88669700 & 7.00248200 \\
\hline $\mathrm{Cl}$ & -8.23191100 & -5.56696100 & 3.44057100 \\
\hline $\mathrm{Cl}$ & -7.83829500 & -6.10872700 & -3.44057100 \\
\hline $\mathrm{Cl}$ & -6.54435500 & -4.32777200 & -7.00248200 \\
\hline $\mathrm{Cl}$ & -7.83829500 & -0.34543500 & -7.00248200 \\
\hline $\mathrm{Cl}$ & -2.09363800 & -2.88164600 & -9.89473300 \\
\hline $\mathrm{Cl}$ & -3.38757800 & 1.10069100 & -9.89473300 \\
\hline $\mathrm{Cl}$ & 0.00000000 & 3.56191000 & -9.89473300 \\
\hline $\mathrm{Cl}$ & 6.13827200 & 4.88669700 & -7.00248200 \\
\hline $\mathrm{Cl}$ & 2.75069400 & 7.34791600 & -7.00248200 \\
\hline $\mathrm{Cl}$ & 2.75069400 & 9.54929800 & -3.44057100 \\
\hline $\mathrm{Cl}$ & 3.38757800 & 9.34236200 & 3.44057100 \\
\hline $\mathrm{Cl}$ & 8.23191100 & 5.56696100 & -3.44057100 \\
\hline $\mathrm{Cl}$ & 6.13827200 & 8.44860700 & -1.23919000 \\
\hline $\mathrm{Cl}$ & 9.93193300 & 3.22708100 & 1.23919000 \\
\hline $\mathrm{Cl}$ & 7.83829500 & 6.10872700 & 3.44057100 \\
\hline $\mathrm{Cl}$ & 2.09363800 & 7.56140700 & 7.00248200 \\
\hline $\mathrm{Cl}$ & -2.09363800 & 7.56140700 & 7.00248200 \\
\hline $\mathrm{Cl}$ & 2.09363800 & 2.88164600 & 9.89473300 \\
\hline $\mathrm{Cl}$ & -2.09363800 & 2.88164600 & 9.89473300 \\
\hline $\mathrm{Cl}$ & -3.38757800 & -1.10069100 & 9.89473300 \\
\hline $\mathrm{Cl}$ & -2.75069400 & -7.34791600 & 7.00248200 \\
\hline $\mathrm{Cl}$ & -2.75069400 & -9.54929800 & 3.44057100 \\
\hline $\mathrm{Cl}$ & -6.13827200 & -8.44860700 & 1.23919000 \\
\hline $\mathrm{Cl}$ & 0.00000000 & 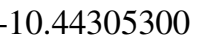 & -1.23919000 \\
\hline
\end{tabular}




\begin{tabular}{|c|c|c|c|}
\hline $\mathrm{Cl}$ & -3.38757800 & -9.34236200 & -3.44057100 \\
\hline $\mathrm{Cl}$ & -2.09363800 & -7.56140700 & -7.00248200 \\
\hline $\mathrm{Cl}$ & 2.09363800 & -2.88164600 & -9.89473300 \\
\hline $\mathrm{Cl}$ & 3.38757800 & 1.10069100 & -9.89473300 \\
\hline $\mathrm{Cl}$ & 6.54435500 & -4.32777200 & -7.00248200 \\
\hline $\mathrm{Cl}$ & 7.83829500 & -0.34543500 & -7.00248200 \\
\hline $\mathrm{Cl}$ & 9.93193300 & 0.33483000 & -3.44057100 \\
\hline $\mathrm{Cl}$ & 9.93193300 & -0.33483000 & 3.44057100 \\
\hline $\mathrm{Cl}$ & 7.83829500 & 0.34543500 & 7.00248200 \\
\hline $\mathrm{Cl}$ & 6.54435500 & 4.32777200 & 7.00248200 \\
\hline $\mathrm{Cl}$ & 3.38757800 & -1.10069100 & 9.89473300 \\
\hline $\mathrm{Cl}$ & 0.00000000 & -3.56191000 & 9.89473300 \\
\hline $\mathrm{Cl}$ & 6.13827200 & -4.88669700 & 7.00248200 \\
\hline $\mathrm{Cl}$ & 2.75069400 & -7.34791600 & 7.00248200 \\
\hline $\mathrm{Cl}$ & 3.38757800 & -9.34236200 & -3.44057100 \\
\hline $\mathrm{Cl}$ & 2.09363800 & -7.56140700 & -7.00248200 \\
\hline $\mathrm{Cl}$ & 7.83829500 & -6.10872700 & -3.44057100 \\
\hline $\mathrm{Cl}$ & 9.93193300 & -3.22708100 & -1.23919000 \\
\hline $\mathrm{Cl}$ & 6.13827200 & -8.44860700 & 1.23919000 \\
\hline $\mathrm{Cl}$ & 2.75069400 & -9.54929800 & 3.44057100 \\
\hline $\mathrm{Cl}$ & 8.23191100 & -5.56696100 & 3.44057100 \\
\hline $\mathrm{Cl}$ & 5.90935500 & -8.13353000 & -6.21346400 \\
\hline $\mathrm{Cl}$ & 5.90935500 & -1.92006600 & -10.05359600 \\
\hline $\mathrm{Cl}$ & 9.56153800 & 3.10673200 & -6.21346400 \\
\hline $\mathrm{Cl}$ & 11.81871100 & 0.00000000 & 0.00000000 \\
\hline $\mathrm{Cl}$ & 9.56153800 & -6.94686400 & 0.00000000 \\
\hline $\mathrm{Cl}$ & 0.00000000 & -6.21346400 & -10.05359600 \\
\hline $\mathrm{Cl}$ & -5.90935500 & -1.92006600 & -10.05359600 \\
\hline $\mathrm{Cl}$ & -3.65218200 & 5.02679800 & -10.05359600 \\
\hline $\mathrm{Cl}$ & 3.65218200 & 5.02679800 & -10.05359600 \\
\hline $\mathrm{Cl}$ & -5.90935500 & -8.13353000 & -6.21346400 \\
\hline $\mathrm{Cl}$ & -3.65218200 & -11.24026200 & 0.00000000 \\
\hline $\mathrm{Cl}$ & 3.65218200 & -11.24026200 & 0.00000000 \\
\hline $\mathrm{Cl}$ & 3.65218200 & -5.02679800 & 10.05359600 \\
\hline $\mathrm{Cl}$ & 9.56153800 & -3.10673200 & 6.21346400 \\
\hline $\mathrm{Cl}$ & 0.00000000 & -10.05359600 & 6.21346400 \\
\hline $\mathrm{Cl}$ & 9.56153800 & 6.94686400 & 0.00000000 \\
\hline $\mathrm{Cl}$ & 5.90935500 & 1.92006600 & 10.05359600 \\
\hline $\mathrm{Cl}$ & 5.90935500 & 8.13353000 & 6.21346400 \\
\hline $\mathrm{Cl}$ & 0.00000000 & 6.21346400 & 10.05359600 \\
\hline $\mathrm{Cl}$ & -5.90935500 & 8.13353000 & 6.21346400 \\
\hline $\mathrm{Cl}$ & -3.65218200 & 11.24026200 & 0.00000000 \\
\hline $\mathrm{Cl}$ & -9.56153800 & 6.94686400 & 0.00000000 \\
\hline $\mathrm{Cl}$ & -9.56153800 & -6.94686400 & 0.00000000 \\
\hline $\mathrm{Cl}$ & -9.56153800 & -3.10673200 & 6.21346400 \\
\hline $\mathrm{Cl}$ & -11.81871100 & 0.00000000 & 0.00000000 \\
\hline $\mathrm{Cl}$ & -5.90935500 & 1.92006600 & 10.05359600 \\
\hline $\mathrm{Cl}$ & -9.56153800 & 3.10673200 & -6.21346400 \\
\hline $\mathrm{Cl}$ & 3.65218200 & 11.24026200 & 0.00000000 \\
\hline $\mathrm{Cl}$ & -3.65218200 & -5.02679800 & 10.05359600 \\
\hline $\mathrm{Cl}$ & 0.00000000 & 10.05359600 & -6.21346400 \\
\hline $\mathrm{Cl}$ & 2.58966400 & -3.56436700 & 7.12873400 \\
\hline $\mathrm{Cl}$ & 4.19016500 & 1.36146700 & 7.12873400 \\
\hline $\mathrm{Cl}$ & 6.77982900 & -2.20290000 & 4.40580000 \\
\hline $\mathrm{Cl}$ & 8.38032900 & 0.00000000 & 0.00000000 \\
\hline $\mathrm{Cl}$ & 6.77982900 & -4.92583400 & 0.00000000 \\
\hline $\mathrm{Cl}$ & 4.19016500 & -5.76726700 & -4.40580000 \\
\hline $\mathrm{Cl}$ & 2.58966400 & -7.97016700 & 0.00000000 \\
\hline $\mathrm{Cl}$ & -2.58966400 & -7.97016700 & 0.00000000 \\
\hline $\mathrm{Cl}$ & 0.00000000 & -7.12873400 & 4.40580000 \\
\hline $\mathrm{Cl}$ & -2.58966400 & -3.56436700 & 7.12873400 \\
\hline
\end{tabular}




$\begin{array}{lrrr}\mathrm{Cl} & -6.77982900 & -2.20290000 & 4.40580000 \\ \mathrm{Cl} & -4.19016500 & 1.36146700 & 7.12873400 \\ \mathrm{Cl} & -6.77982900 & -4.92583400 & 0.00000000 \\ \mathrm{Cl} & -8.38032900 & 0.00000000 & 0.00000000 \\ \mathrm{Cl} & -6.77982900 & 2.20290000 & -4.40580000 \\ \mathrm{Cl} & -6.77982900 & 4.92583400 & 0.00000000 \\ \mathrm{Cl} & -4.19016500 & -1.36146700 & -7.12873400 \\ \mathrm{Cl} & -2.58966400 & 3.56436700 & -7.12873400 \\ \mathrm{Cl} & -4.19016500 & -5.76726700 & -4.40580000 \\ \mathrm{Cl} & 0.00000000 & -4.40580000 & -7.12873400 \\ \mathrm{Cl} & 4.19016500 & -1.36146700 & -7.12873400 \\ \mathrm{Cl} & 6.77982900 & 4.92583400 & 0.00000000 \\ \mathrm{Cl} & 6.77982900 & 2.20290000 & -4.40580000 \\ \mathrm{Cl} & 4.19016500 & 5.76726700 & 4.40580000 \\ \mathrm{Cl} & 2.58966400 & 7.97016700 & 0.00000000 \\ \mathrm{Cl} & -2.58966400 & 7.97016700 & 0.00000000 \\ \mathrm{Cl} & 0.00000000 & 7.12873400 & -4.40580000 \\ \mathrm{Cl} & 2.58966400 & 3.56436700 & -7.12873400 \\ \mathrm{Cl} & -4.19016500 & 5.76726700 & 4.40580000 \\ \mathrm{Cl} & 0.00000000 & 4.40580000 & 7.12873400\end{array}$

Output coordinates:

$\begin{array}{lrrr}\mathrm{Mg} & 7.394769 & -6.434510 & 1.390039 \\ \mathrm{Mg} & 3.834473 & -9.021217 & 1.390039 \\ \mathrm{Mg} & 2.200384 & -9.552165 & -1.390039 \\ \mathrm{Mg} & 3.560296 & -7.680406 & -5.133556 \\ \mathrm{Mg} & 6.204308 & -5.759419 & -5.133556 \\ \mathrm{Mg} & 8.404692 & -5.044471 & -1.390039 \\ \mathrm{Mg} & 7.394769 & -4.120890 & 5.133556 \\ \mathrm{Mg} & 3.834473 & -5.277700 & 7.447177 \\ \mathrm{Mg} & 1.634089 & -8.306269 & 5.133556 \\ \mathrm{Mg} & -1.634090 & -8.306269 & 5.133556 \\ \mathrm{Mg} & -2.200385 & -9.552164 & -1.390039 \\ \mathrm{Mg} & -0.000000 & -6.523596 & -7.447177 \\ \mathrm{Mg} & -0.000000 & -3.743517 & -9.165360 \\ \mathrm{Mg} & 3.560296 & -1.156811 & -9.165360 \\ \mathrm{Mg} & 6.204308 & -2.015902 & -7.447177 \\ \mathrm{Mg} & 8.404692 & 1.012667 & -5.133556 \\ \mathrm{Mg} & 9.764604 & -0.859092 & -1.390039 \\ \mathrm{Mg} & 9.764604 & 0.859091 & 1.390039 \\ \mathrm{Mg} & 8.404692 & -1.012667 & 5.133556 \\ \mathrm{Mg} & 2.200384 & -3.028569 & 9.165360 \\ \mathrm{Mg} & 3.560296 & 1.156810 & 9.165360 \\ \mathrm{Mg} & 6.204308 & 2.015902 & 7.447177 \\ \mathrm{Mg} & 6.204308 & 5.759419 & 5.133556 \\ \mathrm{Mg} & 8.404693 & 5.044470 & 1.390039 \\ \mathrm{Mg} & 7.394770 & 6.434510 & -1.390039 \\ \mathrm{Mg} & 7.394770 & 4.120889 & -5.133556 \\ \mathrm{Mg} & 3.834474 & 5.277700 & -7.447177 \\ \mathrm{Mg} & 2.200384 & 3.028569 & -9.165360 \\ \mathrm{Mg} & -3.560297 & -7.680406 & -5.133556 \\ \mathrm{Mg} & -7.394769 & 4.120890 & -5.133556 \\ \mathrm{Mg} & -8.404692 & 1.012667 & -5.133556 \\ \mathrm{Mg} & -9.764604 & -0.859091 & -1.390039 \\ \mathrm{Mg} & -9.764604 & 0.859092 & 1.390039 \\ \mathrm{Mg} & -8.404692 & 5.044471 & 1.390039 \\ \mathrm{Mg} & -3.834473 & 9.021217 & -1.390039 \\ \mathrm{Mg} & -1.634089 & 8.306269 & -5.133556 \\ \mathrm{Mg} & -3.834473 & 5.277700 & -7.447177 \\ \mathrm{Mg} & -2.200384 & 3.028569 & -9.165360 \\ \mathrm{Mg} & -3.560296 & -1.156810 & -9.165360 \\ \mathrm{Mg} & -6.204308 & -2.015902 & -7.447177\end{array}$




\begin{tabular}{|c|c|c|c|}
\hline $\mathrm{Mg}$ & -8.404693 & -5.044470 & -1.390039 \\
\hline $\mathrm{Mg}$ & -7.394770 & -6.434510 & 1.390039 \\
\hline $\mathrm{Mg}$ & -7.394770 & -4.120889 & 5.133556 \\
\hline $\mathrm{Mg}$ & -8.404692 & -1.012667 & 5.133556 \\
\hline $\mathrm{Mg}$ & -6.204308 & 2.015902 & 7.447177 \\
\hline $\mathrm{Mg}$ & -6.204308 & 5.759419 & 5.133556 \\
\hline $\mathrm{Mg}$ & -3.560296 & 7.680406 & 5.133556 \\
\hline $\mathrm{Mg}$ & -2.200384 & 9.552165 & 1.390039 \\
\hline $\mathrm{Mg}$ & 1.634090 & 8.306269 & -5.133556 \\
\hline $\mathrm{Mg}$ & 3.834474 & 9.021217 & -1.390039 \\
\hline $\mathrm{Mg}$ & 2.200385 & 9.552164 & 1.390039 \\
\hline $\mathrm{Mg}$ & 3.560297 & 7.680406 & 5.133556 \\
\hline $\mathrm{Mg}$ & 0.000000 & 6.523596 & 7.447177 \\
\hline $\mathrm{Mg}$ & 0.000000 & 3.743517 & 9.165360 \\
\hline $\mathrm{Mg}$ & -3.560296 & 1.156811 & 9.165360 \\
\hline $\mathrm{Mg}$ & -2.200384 & -3.028569 & 9.165360 \\
\hline $\mathrm{Mg}$ & -3.834474 & -5.277700 & 7.447177 \\
\hline $\mathrm{Mg}$ & -6.204308 & -5.759419 & -5.133556 \\
\hline $\mathrm{Mg}$ & -3.834474 & -9.021217 & 1.390039 \\
\hline $\mathrm{Mg}$ & -7.394769 & 6.434510 & -1.390039 \\
\hline $\mathrm{Cl}$ & -6.106333 & 5.156249 & -6.818556 \\
\hline $\mathrm{Cl}$ & -8.015693 & 5.776638 & -3.570158 \\
\hline $\mathrm{Cl}$ & -7.970894 & 5.838298 & 3.570158 \\
\hline $\mathrm{Cl}$ & -9.880254 & 3.210290 & 1.562538 \\
\hline $\mathrm{Cl}$ & -9.880254 & -3.210289 & -1.562538 \\
\hline $\mathrm{Cl}$ & -9.880254 & 0.038109 & -3.570158 \\
\hline $\mathrm{Cl}$ & -3.016923 & 7.400836 & -6.818556 \\
\hline $\mathrm{Cl}$ & -3.016923 & 9.408456 & -3.570158 \\
\hline $\mathrm{Cl}$ & -6.106333 & 8.404647 & -1.562538 \\
\hline $\mathrm{Cl}$ & 0.000000 & 10.388714 & 1.562538 \\
\hline $\mathrm{Cl}$ & -3.089409 & 9.384904 & 3.570158 \\
\hline $\mathrm{Cl}$ & -6.790844 & 4.214100 & 6.818556 \\
\hline $\mathrm{Cl}$ & -7.970894 & 0.582281 & 6.818556 \\
\hline $\mathrm{Cl}$ & -9.880254 & -0.038108 & 3.570158 \\
\hline $\mathrm{Cl}$ & -6.106333 & -5.156248 & 6.818556 \\
\hline $\mathrm{Cl}$ & -8.015693 & -5.776637 & 3.570158 \\
\hline $\mathrm{Cl}$ & -7.970894 & -5.838298 & -3.570158 \\
\hline $\mathrm{Cl}$ & -6.790845 & -4.214099 & -6.818556 \\
\hline $\mathrm{Cl}$ & -7.970894 & -0.582280 & -6.818556 \\
\hline $\mathrm{Cl}$ & -1.909360 & -2.628009 & -9.990737 \\
\hline $\mathrm{Cl}$ & -3.089410 & 1.003810 & -9.990737 \\
\hline $\mathrm{Cl}$ & 0.000000 & 3.248398 & -9.990737 \\
\hline $\mathrm{Cl}$ & 6.106333 & 5.156248 & -6.818556 \\
\hline $\mathrm{Cl}$ & 3.016924 & 7.400836 & -6.818556 \\
\hline $\mathrm{Cl}$ & 3.016924 & 9.408456 & -3.570158 \\
\hline $\mathrm{Cl}$ & 3.089410 & 9.384904 & 3.570158 \\
\hline $\mathrm{Cl}$ & 8.015693 & 5.776637 & -3.570158 \\
\hline $\mathrm{Cl}$ & 6.106333 & 8.404646 & -1.562538 \\
\hline $\mathrm{Cl}$ & 9.880254 & 3.210289 & 1.562538 \\
\hline $\mathrm{Cl}$ & 7.970894 & 5.838298 & 3.570158 \\
\hline $\mathrm{Cl}$ & 1.909361 & 7.760705 & 6.818556 \\
\hline $\mathrm{Cl}$ & -1.909360 & 7.760705 & 6.818556 \\
\hline $\mathrm{Cl}$ & 1.909360 & 2.628009 & 9.990737 \\
\hline $\mathrm{Cl}$ & -1.909360 & 2.628009 & 9.990737 \\
\hline $\mathrm{Cl}$ & -3.089410 & -1.003810 & 9.990737 \\
\hline $\mathrm{Cl}$ & -3.016924 & -7.400836 & 6.818556 \\
\hline $\mathrm{Cl}$ & -3.016924 & -9.408456 & 3.570158 \\
\hline $\mathrm{Cl}$ & -6.106333 & -8.404646 & 1.562538 \\
\hline $\mathrm{Cl}$ & -0.000000 & -10.388714 & -1.562538 \\
\hline $\mathrm{Cl}$ & -3.089410 & -9.384904 & -3.570158 \\
\hline $\mathrm{Cl}$ & -1.909361 & -7.760705 & -6.88 \\
\hline
\end{tabular}




\begin{tabular}{|c|c|c|c|}
\hline $\mathrm{Cl}$ & 1.909360 & -2.628009 & -9.990737 \\
\hline $\mathrm{Cl}$ & 3.089410 & 1.003810 & -9.990737 \\
\hline $\mathrm{Cl}$ & 6.790844 & -4.214100 & -6.818556 \\
\hline $\mathrm{Cl}$ & 7.970894 & -0.582281 & -6.818556 \\
\hline $\mathrm{Cl}$ & 9.880254 & 0.038108 & -3.570158 \\
\hline $\mathrm{Cl}$ & 9.880254 & -0.038109 & 3.570158 \\
\hline $\mathrm{Cl}$ & 7.970894 & 0.582280 & 6.818556 \\
\hline $\mathrm{Cl}$ & 6.790845 & 4.214099 & 6.818556 \\
\hline $\mathrm{Cl}$ & 3.089410 & -1.003810 & 9.990737 \\
\hline $\mathrm{Cl}$ & -0.000000 & -3.248398 & 9.990737 \\
\hline $\mathrm{Cl}$ & 6.106333 & -5.156249 & 6.818556 \\
\hline $\mathrm{Cl}$ & 3.016923 & -7.400836 & 6.818556 \\
\hline $\mathrm{Cl}$ & 3.089409 & -9.384904 & -3.570158 \\
\hline $\mathrm{Cl}$ & 1.909360 & -7.760705 & -6.818556 \\
\hline $\mathrm{Cl}$ & 7.970894 & -5.838298 & -3.570158 \\
\hline $\mathrm{Cl}$ & 9.880254 & -3.210290 & -1.562538 \\
\hline $\mathrm{Cl}$ & 6.106333 & -8.404647 & 1.562538 \\
\hline $\mathrm{Cl}$ & 3.016923 & -9.408456 & 3.570158 \\
\hline $\mathrm{Cl}$ & 8.015693 & -5.776638 & 3.570158 \\
\hline $\mathrm{Cl}$ & 5.743780 & -7.905636 & -6.039368 \\
\hline $\mathrm{Cl}$ & 5.743780 & -1.866268 & -9.771903 \\
\hline $\mathrm{Cl}$ & 9.293632 & 3.019684 & -6.039368 \\
\hline $\mathrm{Cl}$ & 11.487561 & -0.000001 & 0.000000 \\
\hline $\mathrm{Cl}$ & 9.293632 & -6.752219 & 0.000000 \\
\hline $\mathrm{Cl}$ & -0.000000 & -6.039368 & -9.771903 \\
\hline $\mathrm{Cl}$ & -5.743780 & -1.866267 & -9.771903 \\
\hline $\mathrm{Cl}$ & -3.549851 & 4.885952 & -9.771903 \\
\hline $\mathrm{Cl}$ & 3.549852 & 4.885951 & -9.771903 \\
\hline $\mathrm{Cl}$ & -5.743781 & -7.905635 & -6.039368 \\
\hline $\mathrm{Cl}$ & -3.549852 & -10.925319 & 0.000000 \\
\hline $\mathrm{Cl}$ & 3.549851 & -10.925320 & 0.000000 \\
\hline $\mathrm{Cl}$ & 3.549851 & -4.885952 & 9.771903 \\
\hline $\mathrm{Cl}$ & 9.293632 & -3.019684 & 6.039368 \\
\hline $\mathrm{Cl}$ & -0.000000 & -9.771903 & 6.039368 \\
\hline $\mathrm{Cl}$ & 9.293632 & 6.752218 & 0.000000 \\
\hline $\mathrm{Cl}$ & 5.743780 & 1.866267 & 9.771903 \\
\hline $\mathrm{Cl}$ & 5.743781 & 7.905635 & 6.039368 \\
\hline $\mathrm{Cl}$ & 0.000000 & 6.039368 & 9.771903 \\
\hline $\mathrm{Cl}$ & -5.743780 & 7.905636 & 6.039368 \\
\hline $\mathrm{Cl}$ & -3.549851 & 10.925320 & 0.000000 \\
\hline $\mathrm{Cl}$ & -9.293632 & 6.752219 & 0.000000 \\
\hline $\mathrm{Cl}$ & -9.293632 & -6.752218 & 0.000000 \\
\hline $\mathrm{Cl}$ & -9.293632 & -3.019684 & 6.039368 \\
\hline $\mathrm{Cl}$ & -11.487561 & 0.000001 & 0.000000 \\
\hline $\mathrm{Cl}$ & -5.743780 & 1.866268 & 9.771903 \\
\hline $\mathrm{Cl}$ & -9.293632 & 3.019684 & -6.039368 \\
\hline $\mathrm{Cl}$ & 3.549852 & 10.925319 & 0.000000 \\
\hline $\mathrm{Cl}$ & -3.549852 & -4.885951 & 9.771903 \\
\hline $\mathrm{Cl}$ & 0.000000 & 9.771903 & -6.039368 \\
\hline $\mathrm{Cl}$ & 2.479574 & -3.412842 & 6.825683 \\
\hline $\mathrm{Cl}$ & 4.012036 & 1.303589 & 6.825683 \\
\hline $\mathrm{Cl}$ & 6.491610 & -2.109252 & 4.218504 \\
\hline $\mathrm{Cl}$ & 8.024072 & -0.000000 & 0.000000 \\
\hline $\mathrm{Cl}$ & 6.491610 & -4.716431 & 0.000000 \\
\hline $\mathrm{Cl}$ & 4.012036 & -5.522094 & -4.218504 \\
\hline $\mathrm{Cl}$ & 2.479574 & -7.631346 & 0.000000 \\
\hline $\mathrm{Cl}$ & -2.479575 & -7.631345 & 0.000000 \\
\hline $\mathrm{Cl}$ & -0.000000 & -6.825683 & 4.218504 \\
\hline $\mathrm{Cl}$ & -2.479575 & -3.412841 & 6.825683 \\
\hline $\mathrm{Cl}$ & -6.491610 & -2.109252 & 4.218504 \\
\hline $\mathrm{Cl}$ & -4.012036 & 1.303590 & 6.825683 \\
\hline
\end{tabular}




\begin{tabular}{|c|c|c|c|}
\hline $\mathrm{Cl}$ & -6.491610 & -4.716431 & 0.000000 \\
\hline $\mathrm{Cl}$ & -8.024072 & 0.000000 & 0.000000 \\
\hline $\mathrm{Cl}$ & -6.491610 & 2.109252 & -4.218504 \\
\hline $\mathrm{Cl}$ & -6.491610 & 4.716431 & 0.000000 \\
\hline $\mathrm{Cl}$ & -4.012036 & -1.303589 & -6.825683 \\
\hline $\mathrm{Cl}$ & -2.479574 & 3.412842 & -6.825683 \\
\hline $\mathrm{Cl}$ & -4.012036 & -5.522093 & -4.218504 \\
\hline $\mathrm{Cl}$ & -0.000000 & -4.218504 & -6.825683 \\
\hline $\mathrm{Cl}$ & 4.012036 & -1.303590 & -6.825683 \\
\hline $\mathrm{Cl}$ & 6.491610 & 4.716431 & 0.000000 \\
\hline $\mathrm{Cl}$ & 6.491610 & 2.109252 & -4.218504 \\
\hline $\mathrm{Cl}$ & 4.012036 & 5.522093 & 4.218504 \\
\hline $\mathrm{Cl}$ & 2.479575 & 7.631345 & 0.000000 \\
\hline $\mathrm{Cl}$ & -2.479574 & 7.631346 & 0.000000 \\
\hline $\mathrm{Cl}$ & 0.000000 & 6.825683 & -4.218504 \\
\hline $\mathrm{Cl}$ & 2.479575 & 3.412841 & -6.825683 \\
\hline $\mathrm{Cl}$ & -4.012036 & 5.522094 & 4.218504 \\
\hline $\mathrm{Cl}$ & 0.000000 & 4.218504 & 6.825683 \\
\hline
\end{tabular}

